EPJ manuscript No.

(will be inserted by the editor)

\title{
Deformation change in light iridium nuclei from laser spectroscopy
}

D. Verney ${ }^{1}$, L. Cabaret $^{2}$, J.E. Crawford ${ }^{3}$, H.T. Duong ${ }^{2}$, B. Fricke ${ }^{7}$, J. Genevey ${ }^{4}$, G. Huber ${ }^{5}$, F. Ibrahim ${ }^{4}$ a, M. Krieg $^{5}$, F. Le Blanc ${ }^{1}$, J.K.P. Lee ${ }^{3}$, G. Le Scornet ${ }^{6}$, D. Lunney ${ }^{6}$, J. Obert ${ }^{1}$, J. Oms ${ }^{1}$, J. Pinard ${ }^{2}$, J.C. Puteaux ${ }^{1}$, K. Rashid $^{7}$, B. Roussière ${ }^{1}$, J. Sauvage ${ }^{1}$, V. Sebastian ${ }^{5}$, and the ISOLDE Collaboration ${ }^{8}$

${ }^{1}$ Institut de Physique Nucléaire, IN2P3-CNRS/Université Paris Sud-XI, F-91406 Orsay Cedex, France

2 Laboratoire Aimé Cotton, CNRS, F-91405 Orsay Cedex, France

${ }^{3}$ Physics Department, McGill University, Montréal, Canada H3A2T8

${ }^{4}$ Laboratoire de Physique Subatomique et de Cosmologie, IN2P3-CNRS/Université Joseph Fourier-Grenoble I, F-38026 Grenoble Cedex, France

${ }^{5}$ Institut für Physik der Universität Mainz, D-55099 Mainz, Germany

${ }^{6}$ Centre de Spectrométrie Nucléaire et de Spectrométrie de Masse, IN2P3-CNRS/Université Paris Sud-XI, F-91405 Orsay Cedex, France

7 Institut für Theoretische Physik der Universität Kassel, D-34109 Kassel, Germany

${ }^{8}$ CERN, CH-1211 Genève 23, Switzerland

Received: date / Revised version: date

\begin{abstract}
Laser spectroscopy measurements have been performed on neutron-deficient and stable Ir isotopes using the COMPLIS experimental setup installed at ISOLDE-CERN. The radioactive Ir atoms were obtained from successive decays of a mass separated $\mathrm{Hg}$ beam deposited onto a carbon substrate after deceleration to $1 \mathrm{kV}$ and subsequently laser desorbed. A three-color, two-step resonant scheme was used to selectively ionize the desorbed Ir atoms. The hyperfine structure (HFS) and isotope shift (IS) of the first transition of the ionization path $5 d^{7} 6 s^{2}{ }^{4} F_{9 / 2} \rightarrow 5 d^{7} 6 s 6 p{ }^{6} F_{11 / 2}$ at $351.5 \mathrm{~nm}$ were measured for ${ }^{182-189} \mathrm{Ir}$, ${ }^{186} \mathrm{Ir}^{m}$ and the stable ${ }^{191,193} \mathrm{Ir}$. The nuclear magnetic moments $\mu_{I}$ and the spectroscopic quadrupole moments $Q_{s}$ were obtained from the HFS spectra and the change of the mean square charge radii from the IS measurements. The sign of $\mu_{I}$ was experimentally determined for the first time for the masses $182 \leq \mathrm{A} \leq 189$ and the isomeric state ${ }^{186} \mathrm{Ir}^{m}$. The spectroscopic quadrupole moments of ${ }^{182} \mathrm{Ir}$ and ${ }^{183} \mathrm{Ir}$ were measured also for the first time. A large mean square charge radius change between ${ }^{187} \mathrm{Ir}$ and ${ }^{186} \mathrm{Ir}^{g}$ and between ${ }^{186} \mathrm{Ir}^{m}$ and ${ }^{186} \mathrm{Ir}^{g}$ was observed corresponding to a sudden increase in deformation: from $\beta_{2} \simeq+0.16$ for the heavier group $A=193,191,189,187$ and $186 \mathrm{~m}$ to $\beta_{2} \geq+0.2$ for the lighter group $A=186 \mathrm{~g}, 185,184$, 183 and 182. These results were analyzed in the framework of a microscopic treatment of an axial rotor plus one or two quasiparticle(s). This sudden deformation change is associated with a change in the proton state that describes the odd-nuclei ground state or that participates in the coupling with the neutron in the odd-odd nuclei. This state is identified with the $\pi 3 / 2^{+}$[402] orbital for the heavier group and with the $\pi 1 / 2^{-}[541]$ orbital stemming from the $1 h_{9 / 2}$ spherical subshell for the lighter group. That last state seems to affect strongly the observed values of the nuclear moments.
\end{abstract}

PACS. 21.10.Ky Electromagnetic moments - 21.10.Hw Spin,parity and isobaric spin - 21.60.Ev Collective models $-27.70 .+\mathrm{q} 150 \leq A \leq 189-42.62$.Fi Laser spectroscopy

\section{Introduction}

The nuclear region which extends from the rare earth nuclei to the lead isotopes and especially the neutron deficient $\mathrm{Hg}, \mathrm{Pt}$ and $\mathrm{Au}$ nuclei - the so-called transitional region - is well known for providing a wealth of experimental evidence for shape coexistence and transitions. From

\footnotetext{
a Permanent address: Institut de Physique Nucléaire, IN2P3-CNRS/Université Paris Sud-XI, F-91406 Orsay Cedex, France
}

the historical discovery of the dramatic shape transitions between the odd and even neutron deficient $\mathrm{Hg}$ nuclei from optical isotope shift measurements [1,2] it appeared that information provided by optical and laser techniques were to be of major importance in understanding key features of the structure of the nuclei in this region. In particular, some important questions arose concerning the interplay between the collective and individual degrees of freedom when the neutron number approaches the midshell number $\mathrm{N}=104$ while the proton configurations are still domi- 
nated by a few holes in the $\mathrm{Z}=50-82$ major shell. In many cases the information provided by isotope shift measurements (the nuclear radii and deformation changes) allied to the information provided by hyperfine structure measurements (the magnetic dipole and electric quadrupole moments) were of crucial interest in order to give a clearer interpretation of the level scheme patterns observed in $\gamma$ ray spectroscopy. As will be seen, this complementarity between an important sum of knowledge coming from $\gamma$ ray spectroscopy and our new measurements coming from on-line laser spectroscopy is the keystone of this work on the neutron-deficient iridium nuclei.

Contrary to the large deformation changes observed between the odd-even and even-even $\mathrm{Hg}$ and $\mathrm{Pt}$ nuclei, in the case of the odd-proton $\mathrm{Au}$ isotopes a deformation jump occurs only once, between $\mathrm{A}=187$ and $\mathrm{A}=186$ and everything happens as if the unpaired proton provided a kind of stabilization of the deformation [3]. Besides, Seewald et al. showed an important difference in the axial deformation parameters between the ground and isomeric state of ${ }^{186}$ Ir from quadrupole moment measurements [4]. Therefore it is interesting to see whether a shape transition or jump in deformation occurs in another odd-proton isotopic chain like Ir with two protons less than $\mathrm{Au}$.

To answer this question, an on-line laser spectroscopy experiment was performed using the COMPLIS (COllaboration for spectroscopic Measurements using a Pulsed Laser Ion Source) setup at ISOLDE-CERN. The two main experimental principles which are underlying the COMPLIS setup are resonance ionization mass spectroscopy (RIMS) and pulsed-laser induced desorption (PLID). Most laser spectroscopy studies of radioactive nuclei have been performed using radioactive beams produced with the ISOL method. This is due essentially to the high intensity and high optical quality of the ion beams which are available at ISOL-type facilities. On the other hand, with the ISOL technique, production yields are very sensitive to both the characteristics of the target-ion source ensemble and the physico-chemical properties of the element to be extracted and ionized. The temperature of the target is one of the most critical parameters because it governs the speed and trajectory of the radioactive atoms (via diffusion, effusion and other processes) inside the target ion source ensemble. For the elements characterized by high melting and boiling points this temperature parameter may even inhibit significant production unless very special care is taken. This is the case for the elements of the Pt region which, as can be seen in Fig. 1, are situated next to the Ta-W-Re group characterized by the highest melting and boiling points in nature. Our method consists of taking advantage of the very low melting and boiling points of $\mathrm{Hg}$ (see Fig. 1) and waiting for their $\beta^{+} / E C$ or $\alpha$ decay to successive daughter nuclei $(\mathrm{Au}, \mathrm{Pt}$ etc.), which are then available to produce secondary beams using the PLID and RIMS methods.

The radioactive parent ions are implanted or deposited onto the surface of a carefully selected refractory material. The descendants are then extracted from this material by heating its surface during a very short time using a focused Nd:YAG laser beam. The delay between the end

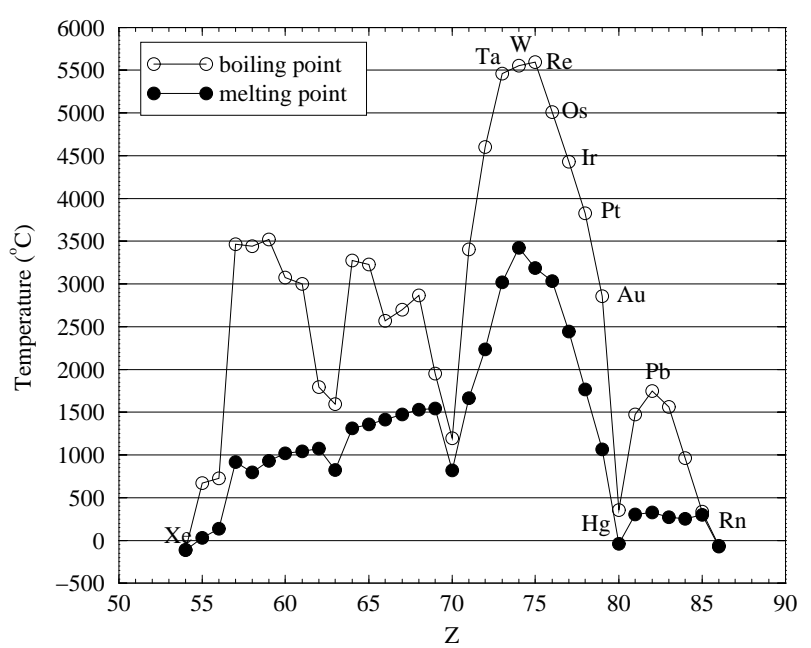

Fig. 1. Melting and boiling points for the elements from Xe to $\operatorname{Rn}[5]$.

of the implantation stage and the heating stage can be controlled. This allows the optimization of the population of the interesting descendants inside the substrate before desorption. Then, the descendant isotopes as a pulsed secondary atomic beam can be efficiently ionized using the RIS process. The technique based on the RIMS following PLID has been applied successfully to the study of the $\mathrm{Au}$ and $\mathrm{Pt}$ nuclei $[3,6-10]$ and more recently with the COMPLIS setup $[11,12]$.

In the first part of this paper we will describe the COMPLIS setup and give details about a few delicate points in the measurement process, especially on the implantation/desorption phase. Then we will present our results on the neutron-deficient isotopes ${ }^{182-189} \mathrm{Ir},{ }^{186} \mathrm{Ir}^{m}$ and the stable ${ }^{191,193} \mathrm{Ir}$ : the mean-square charge radius variation from the isotopic shift and the magnetic dipole moments and spectroscopic quadrupole moments from the hyperfine spectra. In a last part, these results will be analysed using an axial rotor plus one or two quasiparticle model.

\section{Experiment}

The resonant ionization spectroscopy measurement was aimed at exploring the hyperfine structure (HFS) and isotope shift (IS) in the ${ }^{4} F_{9 / 2}\left(0 \mathrm{~cm}^{-1}\right) \rightarrow{ }^{6} F_{11 / 2}(28452.31$ $\mathrm{cm}^{-1}$ ) transition of the iridium at $\lambda=351.5 \mathrm{~nm}$. Iridium belongs to the class of the open d-shell atoms which exhibit spectra characterized by a huge number of lines. In such atoms, the hyperfine structure features are dominated by important relativistic and configuration interaction effects, and the transition had to be carefully selected in order to connect the ground $s^{2}$-state to a $s p$ excited state of reasonable purity to obtain a significant isotope shift. A fit of the fine-structure parameters by S. Büttgenbach et al. $[13,14]$ leads to a very pure $5 d^{7} 6 s^{2}$ configuration for the intermediate coupling wave function of 


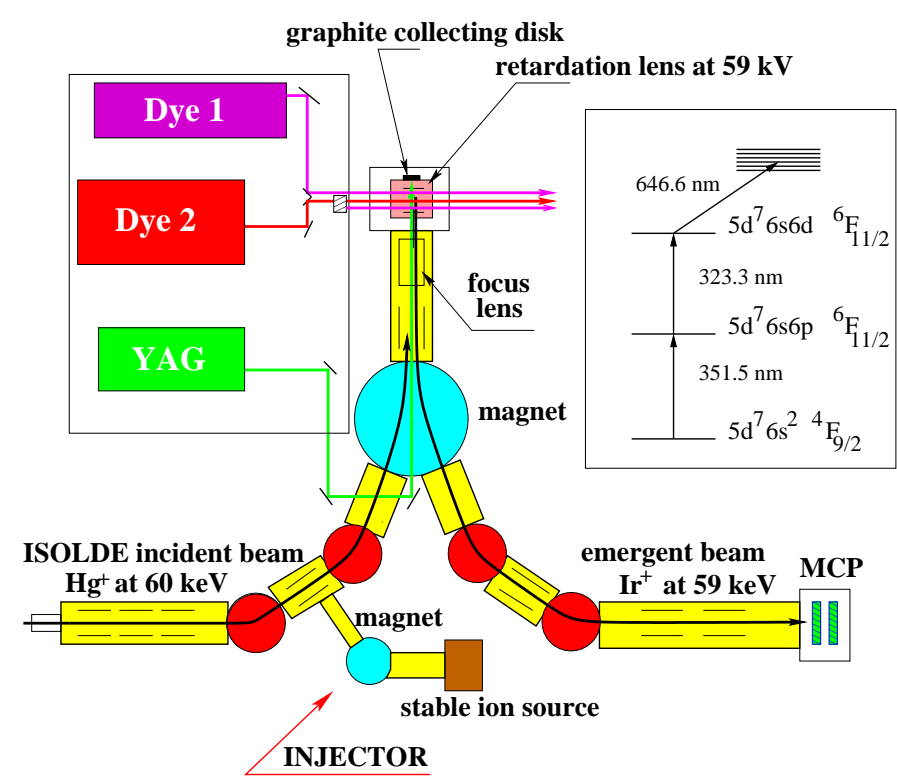

Fig. 2. The COMPLIS setup. The insert shows the ionization scheme used in this experiment.

the ground state whose main components are $84 \%\left(5 d^{7} 6 s^{2}\right.$ $\left.{ }^{4} F_{9 / 2}\right)+14 \%\left(5 d^{7} 6 s^{2}{ }^{2} G_{9 / 2}\right)$. The first excited level was chosen for its purity from calculations of configuration mixing performed by J.F. Wyart [15]. The chosen ${ }^{6} F_{11 / 2}$ level corresponds to $95.8 \%$ of $5 d^{7} 6 s 6 p$ and some weak components of the $5 d^{8} 6 p(0.9 \%)$ and the $5 d^{6} 6 s^{2}(3.3 \%)$ configurations. The complete ionization was achieved through a three colour-three step process, which contains, in our case, two UV transitions (the ionization threshold for the iridium atom is situated rather high at $9.1 \mathrm{eV}$ ) :

$$
\begin{array}{cc}
5 d^{7} 6 s^{2}{ }^{4} F_{9 / 2} \rightarrow 5 d^{7} 6 s 6 p{ }^{6} F_{11 / 2} & \lambda=351.5 \mathrm{~nm} \\
5 d^{7} 6 s 6 p^{6} F_{11 / 2} \rightarrow 5 d^{7} 6 s 6 d^{6} F_{11 / 2} & \lambda=323.3 \mathrm{~nm} \\
5 d^{7} 6 s 6 d^{6} F_{11 / 2} \rightarrow \operatorname{Ir}^{+}+\mathrm{e}^{-}(\text {continuum }) & \lambda=646.6 \mathrm{~nm}
\end{array}
$$

\subsection{Measurement process}

Overall procedure The experimental process leading from the ion production to the effective hyperfine spectrum recording has already been described in ref. [16-18]. The neutron deficient $\mathrm{Hg}$ isotopes are obtained as products of the spallation reaction

$$
{ }_{82}^{A} \mathrm{~Pb}(\mathrm{p}, 3 \mathrm{p} x \mathrm{n}){ }_{80}^{A^{\prime}} \mathrm{Hg}
$$

by bombarding a molten lead target with the $1 \mathrm{GeV}$ staggered proton beam delivered by the PS-Booster [19]. The radioactive $\mathrm{Hg}$ ion beam which has been mass selected through the General Purpose Separator (GPS) of ISOLDE is sent into the incident beam line of the COMPLIS setup. A schematic view of the experimental setup is displayed in Fig. 2. The incident beam is decelerated from its extraction energy of $60 \mathrm{keV}$ down to an implantation energy of $1 \mathrm{keV}$ in the interaction chamber. In this chamber the atoms to be studied are desorbed and ionized. The photoions are extracted and guided in the emergent beam line towards the ion detector.

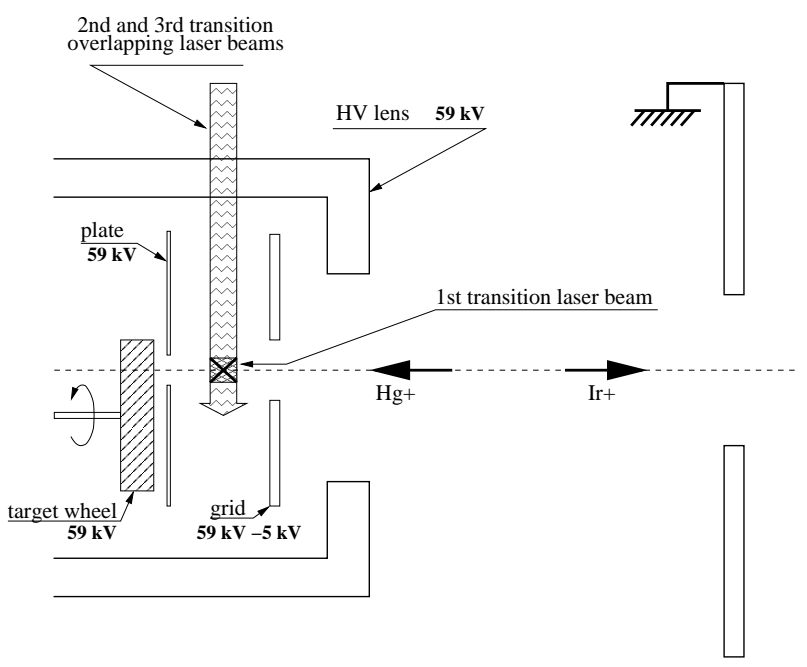

Fig. 3. Schematic view of the interaction chamber of the COMPLIS setup. The graphite substrate is located on a rotating target wheel. The first step laser beam is in the horizontal direction while the second and third transition laser beams are in the vertical direction.

Hyperfine spectrum recording The acceleration/decelaration geometry has been designed in order to provide the deceleration and a strong focusing on the target wheel of the incoming $\mathrm{Hg}$ ion beam. The electrode system is shown in Fig. 3. This system is based on the one described in ref. [20]. It has been shown [21] that $1 \mathrm{keV}$ implantation energy represents an optimum for the ratio of desorbed over implanted atoms that can be obtained by one heat pulse on a graphite substrate. By using some very crude assumptions concerning the diffusion of the implanted atoms inside the graphite substrate, it can be shown that the evolution of the desorption efficiencies measured at COMPLIS by repeated heating of the same ion deposit can be described a very shallow gaussian-like distribution [22]. Thus the main part of the implanted atoms would be confined within a few tenths of an $\AA$ from the graphite surface.

One then waits for an optimized population of descendant isotopes inside the graphite lattice. These descendants are then removed from the lattice in their atomic form by heating the graphite surface with the focused frequencydoubled beam of a pulsed Nd:YAG laser. The repetition rate of this laser as well as that of the whole ionization laser system is $10 \mathrm{~Hz}$. The number of heating laser pulses is set for an optimized desorption efficiency, experimentally determined to be 15 per frequency step, except for ${ }^{182} \mathrm{Ir}$ for which it was 50.

The desorbed atoms are then allowed to diffuse from the target wheel during $12 \mu \mathrm{s}$. The ionization volume is defined by the overlap of three laser beams as shown in Fig. 3. The HFS measurement being performed on the first excitation transition, the first step beam is provided by a high resolution tunable pulsed dye laser.

The complete hyperfine structure is scanned step by step as illustrated in Fig. 4. In a resonant transition between two HFS levels, ions are formed through the three step 


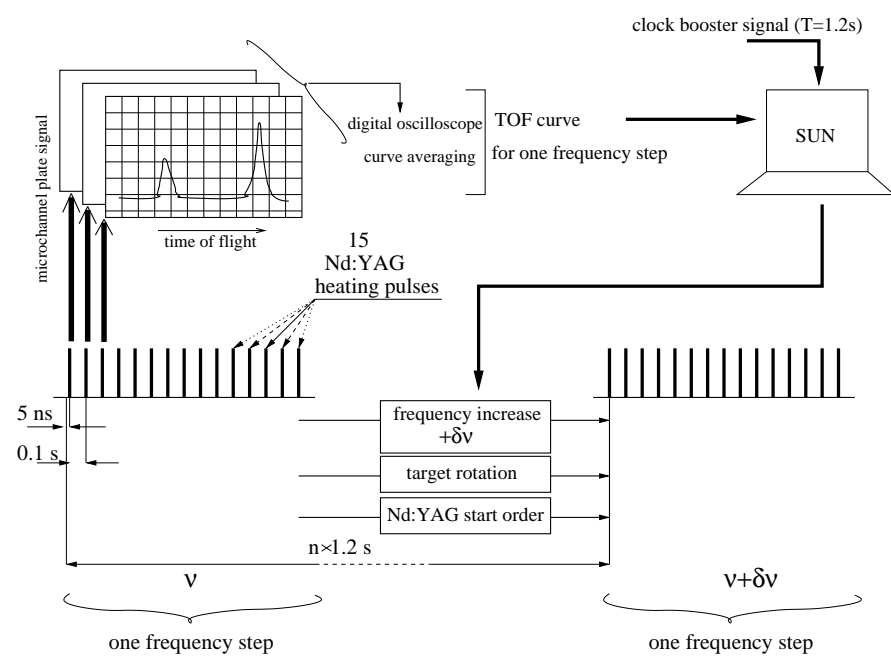

Fig. 4. Recording procedure of a hyperfine spectrum.

process described above, inside the ionization volume. The newly created Ir photo-ions, being submitted to the electric field generated by the $59 \mathrm{kV}$ electrode system, are accelerated towards the emergent beam line of the COMPLIS setup. The photo-ions are detected at the end of the line using microchannel plates. The electrical signal delivered by these plates, which is proportional to the detected ion number, is recorded by a Tektronix TDS540 digital oscilloscope triggered by the ionization laser signal. This allows a measurement of the time of flight of the photoions. Several masses are desorbed at the same time : the stable isotopes which have been previously implanted in order to have a frequency reference and some long-lived radioactive isotopes that would remain from previous implantations on the same graphite zone. The plate and grid allow a pre-acceleration of the photo-ions. This two-field accelerating geometry has been optimized in order to reduce the time spread due to the space distribution of the photo-ions created inside the ionization volume following the prescriptions of ref. [23]. Each recorded curve represents the number of ions detected as a function of the timeof-flight. In our case, 15 (or 50) curves associated with a series of 15 (or 50) heating shot are obtained for each frequency step. Each set of 15 (or 50) curves is summed up by the oscilloscope, and the resulting spectrum is recorded by a SUN workstation. The synchronization of the various operations controlled by the SUN computer is triggered with the $1.2 \mathrm{~s}$ period of the clock of the PS-Booster to make the collections homogeneous.

The whole HFS spectrum is scanned in $60-120 \mathrm{MHz}$ steps. An example of the resulting data matrix of ion number versus time of flight and frequency is shown in Fig. 5. In this figure one can see four HFS spectra corresponding to four different values of the time of flight i.e. to four different masses. The center of gravity of the hyperfine spectrum of the 191 isotope is used as the frequency reference. The isotope shift between mass 191 and mass 187 is shown: it corresponds to the distance in frequency between the centers of gravity, represented by dots, of the

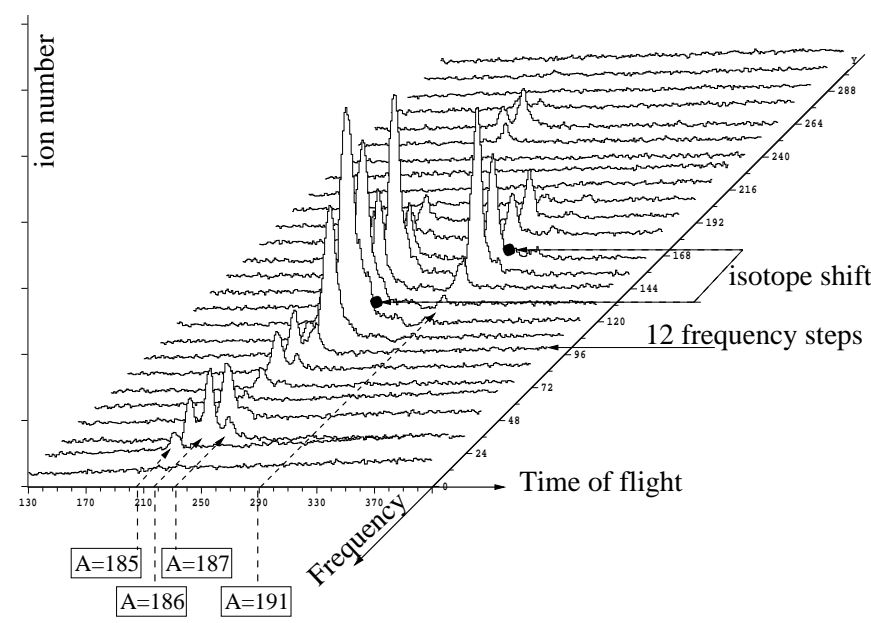

Fig. 5. An example of a data matrix of ion numbers as a function of time of flight and frequency obtained during this experiment. Each time of flight curve which is represented here is actually a sum of 12 recorded time of flight curves i.e. 12 frequency steps.

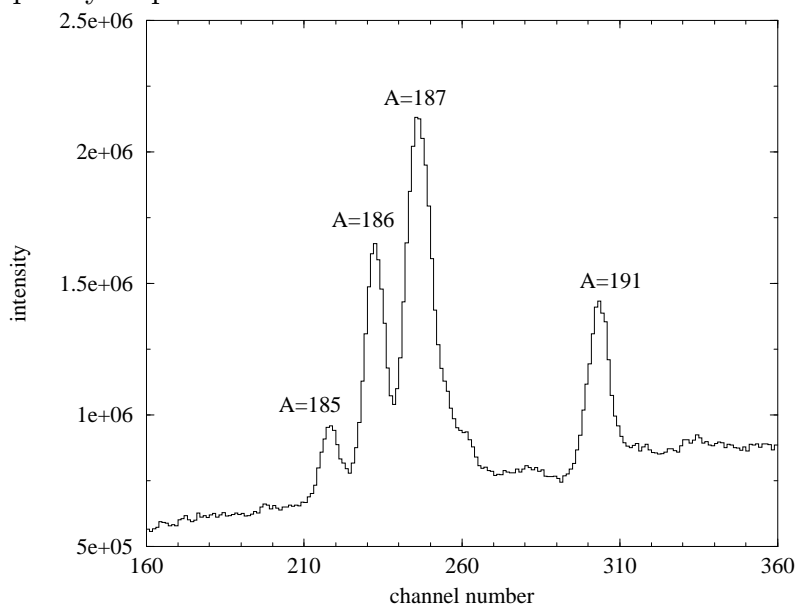

Fig. 6. Total projection of the matrix shown in Fig. 5 on the time of flight axis.

HFS for these two masses. Fig. 6 shows the total projection of the data matrix of Fig. 5 on the time of flight axis. The mass resolving power is about 300 around mass 190 .

Stable isotope injector As no stable Ir beam is available from the ISOLDE target, an ion injector system has been realized. It is composed of an ion source and a magnet for mass selection (Fig. 2). It allows implantations of stable isotopes with controlled quantities to define the optimal running conditions : desorption power, ionization frequencies, timing, determination of the overall efficiency of the system and frequency references for every spectrum recorded on-line.

The laser system The layout of the different lasers composing the COMPLIS system are summarized in Fig. 7. 

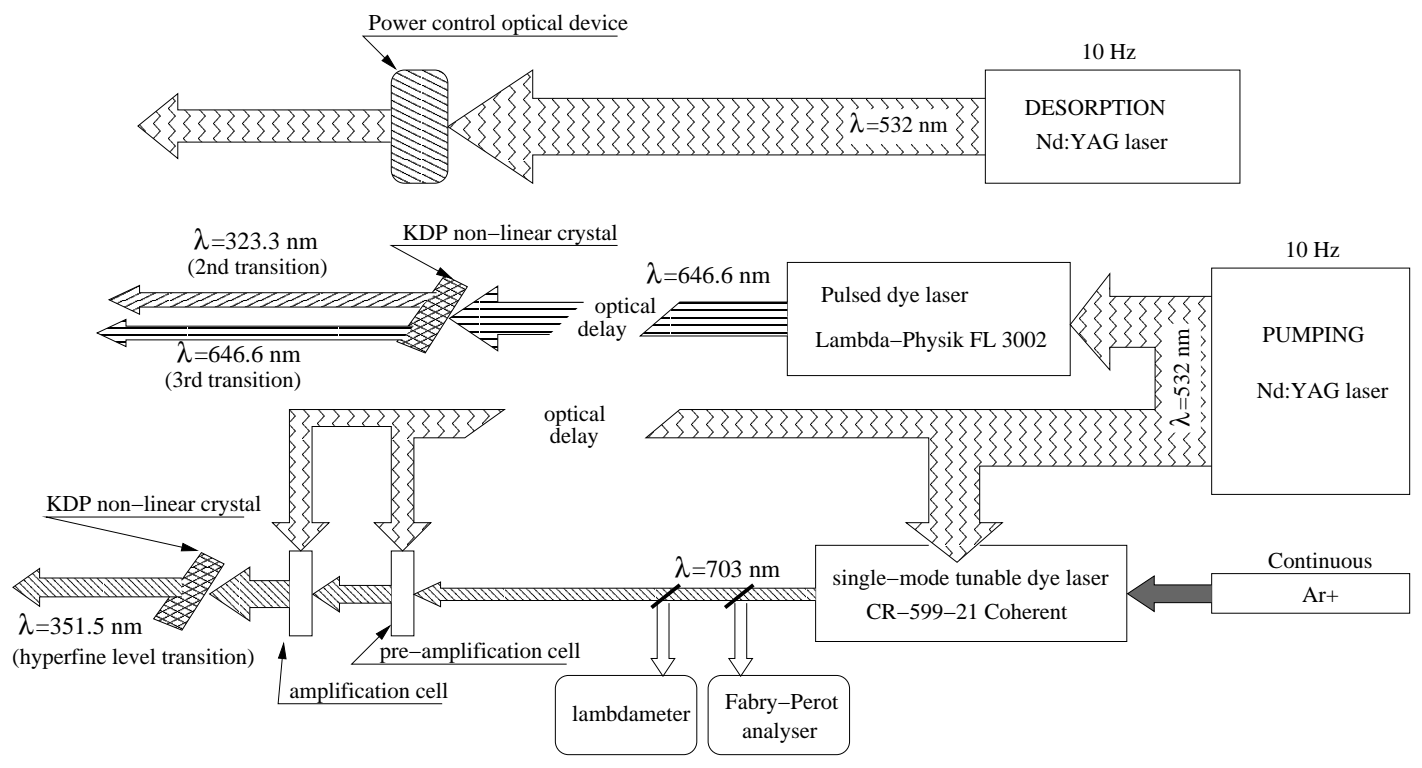

Fig. 7. COMPLIS laser system and optical layout.

The laser system has also been described in ref. [18]. We just mention here that in order to obtain both high resolution and high power (for frequency doubling) for the first excitation step, a pulsed dye laser injection-locked to the frequency of a CW laser is used. Injection-locking is achieved by inserting a supplementary dye cell inside the cavity of a CW high resolution tunable dye laser of the commercial type Coherent Model 599 (this composite system has been called "compulsé" $[24,25])$. The additional cell is pumped by a frequency doubled $(\lambda=532 \mathrm{~nm})$ Nd:YAG laser beam. For the two supplementary ionization steps, a tunable pulsed dye laser of the commercial type Lambda-Physik FL 3002 is used. The beam is frequency doubled via a non-linear crystal and the doubled component $(\lambda=323.3 \mathrm{~nm})$ is used for the second transition while the intense fundamental component $(\lambda=646.6$ $\mathrm{nm}$ ) provides the last transition into the continuum. Such a procedure requires only one laser for two transitions and provides good overlap of the two beams emerging from the crystal.

\subsection{The implantation/waiting/desorption process}

The running modes The target wheel for the implantation - desorption process is shown in Fig. 8. There are two ways of synchronizing the implantation and desorption phases: the so-called implantation/desorption and step by step modes.

The implantation/desorption mode was used for the longer lived isotopes ${ }^{183-189} \mathrm{Ir}$. In this mode, the $\mathrm{Hg}$ ions are implanted while the target wheel is rotating slowly (typically $0.04^{\circ} \cdot \mathrm{s}^{-1}$ ). In order to obtain a uniform deposit, the target is only rotating while the proton beam is on and is stopped as soon as it is off. A typical implantation takes about one hour, when it is over, the target position is brought back to the implantation beginning and both

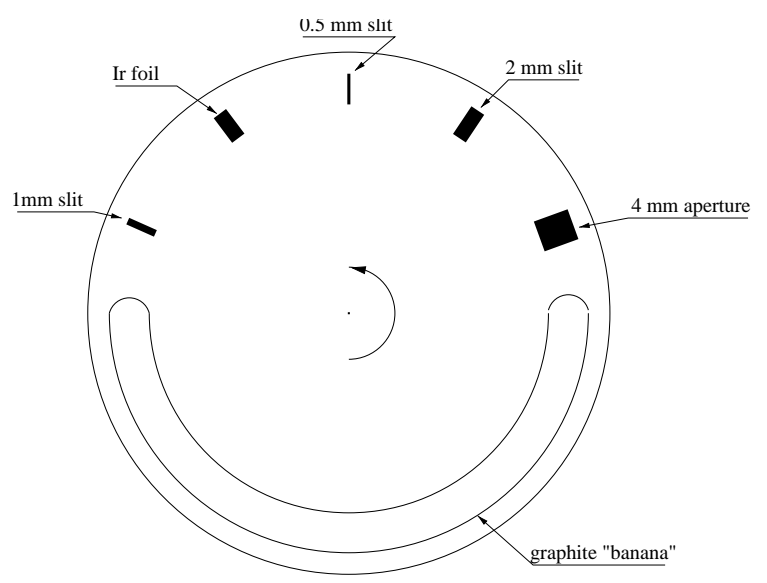

Fig. 8. Front view of the target wheel. To allow implantation desorption on a large area, the graphite surface is shaped into a "banana". Various target positions are left as open apertures so that a Faraday cup can be used to measure beam current passing through. Before the measurements, the incident beam line parameters are set for an optimized current in the $1 \mathrm{~mm}$ slit. Desorption is performed on a Ir foil for settings which need a continuous and large amount of stable Ir isotopes to be available.

the desorption and step by step frequency scan procedures start after a certain delay which depends on the various half-lives in the $\beta$-decay chain $\mathrm{Hg} \rightarrow \mathrm{Au} \rightarrow \mathrm{Pt} \rightarrow \mathrm{Ir}$. The delay between implantation and desorption is the same for every desorption point and corresponds to the collection duration plus the waiting time. The rotation speed is set to provide about $10^{8}$ atoms for each desorption spot.

The ${ }^{182} \mathrm{Hg}$ yield is $8.10^{6}$ atoms $/ \mu \mathrm{C}$, then in order to study ${ }^{182} \mathrm{Ir}$ we had to use the step by step mode which allows some accumulation of the descendant isotopes. The implantation was performed at fixed target position dur- 
ing four PS-Booster macrocycles (one macrocycle was 14.4 $\mathrm{s})$. Since the incident beam line parameters was optimized through the $1 \mathrm{~mm}$ wide slit (see Fig. 8), the implantation distribution had a width of the order of $1 \mathrm{~mm}$. The focusing of the desorption Nd:YAG laser is about 20-50 $\mu \mathrm{m}$ width, the rotation for desorption is set to correspond to the whole implantation spot width for one frequency step (50 heating laser pulses). Then the whole process is repeated from implantation to desorption after incrementing the laser frequency of the value of one step. The delay between implantation and desorption was chosen to be four times the implantation duration (230.4 s). In these conditions about $2.10^{8}$ atoms of ${ }^{182} \mathrm{Ir}$ were available for one frequency step measurement. As each frequency step takes around one minute, a wide HFS scan may last for hours. The HFS is alternately scanned in ascending and descending frequency from one spectrum measurement to another in order to avoid systematic errors.

Influence of the graphite properties on the desorption efficiency The desorption process has a major influence on the overall efficiency of the system. It is in turn strongly determined by the target-graphite properties. Four different species of graphite substrate (Carbone-Lorraine) have been tested on the COMPLIS system, denominated here by 2318, 5890, Pyrolytique Normal (Pyr. N.) and Pyrolytique Transverse (Pyr. T.). The overall efficiencies for each of them (see Tab. 1) are determined as the ratio between the measured photo-ion number over the number of stable Ir ions collected using the stable ion injector. The best efficiency is obtained in the case of a Pyr. N. sample while a factor 2.5 and 5 less is obtained respectively for the 2318 and the 5890 (see Tab. 1). As for Pyr. T. no signal was observed at all. In order to give a qualitative (if not quantitative) explanation for the differences observed one should give an estimate of the surface temperature reached during the heating process. For that purpose we have followed closely the approach of refs. $[21,26]$. A square laser pulse of length $t_{0}=5 \mathrm{~ns}$ is assumed. We suppose that the energy transmission process from the Nd:YAG laser beam to the graphite surface is short enough $(\approx 1 \mathrm{ps})$ as compared to the heating duration $t_{0}$ to be considered as instantaneous and that normal heat diffusion equations apply. Considering a heating penetration depth negligible in front of the heating surface, the geometry of the problem is simplified to the case of a surface heated semi-infinite medium (no propagation parallel to the surface). This hypothesis is verified a posteriori by the results of the calculation.

In those conditions, an estimation of the temperature of the graphite surface during the Nd:YAG laser heating pulse is given by :

$$
T(t)=T_{0}+\frac{2 \Pi_{0}}{K} \sqrt{\frac{\kappa t}{\pi}}
$$

where $t$ denotes the time from the beginning of the pulse, $T_{0}$ is the surface temperature before the pulse, $K$ is the thermal conductivity and $\kappa$ is the diffusivity of the heat. $\Pi_{0}$ is the surface density of heat power, it has been measured to be of the order of $10^{7}$ W.cm ${ }^{-2}$. The values of $K$
Table 1. Thermal properties of the graphite samples: $K$ is the thermal conductivity, $\kappa$ is the diffusivity of heat. As these quantities are temperature dependent, the values estimated for a temperature of $2000 \mathrm{~K}$ (except ${ }^{*}$ : for $800 \mathrm{~K}$ ) which have been used for the calculation are given inside brackets. $\epsilon$ represents the overall efficiency of the COMPLIS set-up measured for the different species.

\begin{tabular}{lllll}
\hline type & name & $\begin{array}{l}K \\
\mathrm{~W} \cdot \mathrm{cm}^{-1} \cdot \mathrm{K}^{-1}\end{array}$ & $\begin{array}{l}\kappa \\
\mathrm{cm}^{2} \cdot \mathrm{s}^{-1}\end{array}$ & $\epsilon$ \\
\hline $\begin{array}{l}\text { poly- } \\
\text { crystalline } \\
\text { poly- } \\
\begin{array}{l}\text { crystalline } \\
\text { pyro- } \\
\text { carbon }\end{array}\end{array}$ & 5890 & $0.9(0.18)$ & $0.657(0.13)$ & $3 \cdot 10^{-7}$ \\
& Normal & $\approx 0.03(0.03)$ & $\approx 0.018(0.018)$ & $1.5 \cdot 10^{-6}$ \\
& $\begin{array}{l}\text { Trans- } \\
\text { verse }\end{array}$ & $\approx 4(0.8)^{*}$ & $\approx 2.42(0.48)^{*}$ & $\approx 0$ \\
\hline
\end{tabular}

and $\kappa$ given by Carbone-Lorraine for the tested graphite samples are reported in Tab. 1. It is important to notice the strong variation (at least one order of magnitude) of these values between the two pyrocarbon samples. It simply reflects the graphite lattice structure anisotropy. The use of the various numerical values reported in Tab. 1 leads to an equivalent surface temperature at the end of the heating pulse of about $1900 \mathrm{~K}$ for the two polycrystalline samples 2318 and 5890. This temperature represents an intermediate value between $980 \mathrm{~K}$ for the Pyr. T. and $3860 \mathrm{~K}$ for the Pyr. N.. These results fit nicely to the qualitative trends of the efficiency observed. As could be expected, the shallower the heating process takes place, the higher the surface temperature is. It has to be noticed however that the only reliable information may come from the differences between the estimated temperatures since the omission of the dissipation along the surface in the calculation may lead to an overestimation of the absolute values. The important point in Eq. 1 is to show that the surface temperature follows linearly the heating power. Therefore, to obtain reliable measurement conditions we have realized a precise Nd:YAG-beam power control device made of a half-wave plate to rotate the laser polarization angle followed by a fixed Glan-Foucault prism as a polarizer; this allows to tune the laser power without modifying the laser parameters and the beam alignment.

\subsection{Interplay between resolution and efficiency}

As usual when using RIMS techniques, resolution and efficiency are closely related on the COMPLIS system and result from a delicate compromise. In order to make this important point clearer we have to consider for simplicity the overlap between the three ionization laser beams inside the pulsed atomic cloud as a parallelepiped with two faces parallel to the graphite surface. The directions and dimensions of such a parallelepiped $(a, b, c)$ are defined in Fig. 9 where the faces $(a, b)$ are parallel to the graphite surface. 


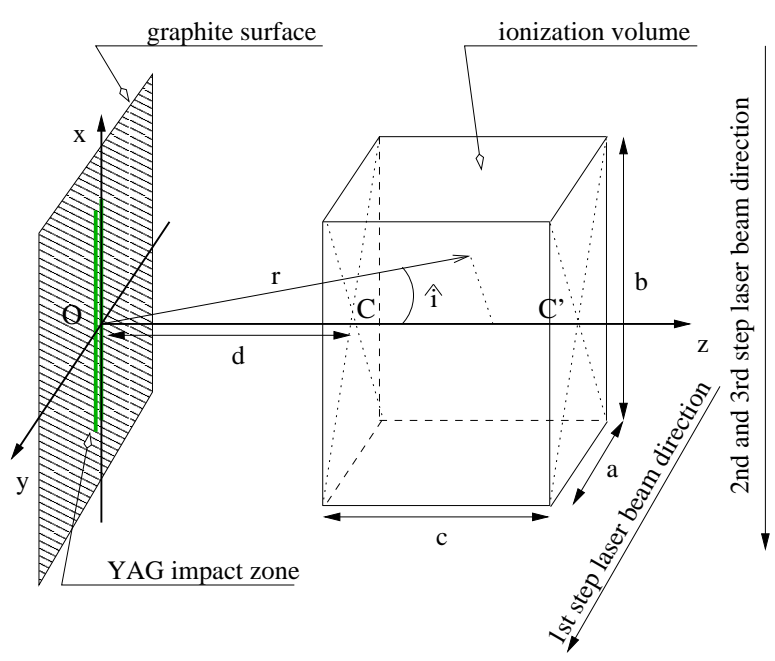

Fig. 9. Geometry of the ionization process: the ionization volume defined by the three laser beam spatial overlap is assumed to be a parallelepiped of sides $(a, b, c)$.

Resolution The Doppler broadening is due to the velocity component of the atoms which is parallel to the direction of the high resolution laser beam providing the hyperfine transitions. The Nd:YAG desorption beam is focused in a $20-50 \mu \mathrm{m}$ wide and $0.5 \mathrm{~cm}$ long segment defining the surface of a pulsed thermal source (see Fig. 9). The ionization volume (defined by $a \simeq 1 \mathrm{~mm}$ and $b=c \simeq 5 \mathrm{~mm}$ ) being far larger, the width of the pulsed atomic source is considered point-like. In these geometrical conditions, the maximum component of velocity of the atoms inside the interaction region along the direction of the hyperfine excitation beam is :

$$
v_{/ / \max }=\frac{a}{2 t_{d}}
$$

where $t_{d}$ is defined as the time separating the desorption pulses from the ionization shots and $a$ the interaction box dimension shown in Fig. 9. The full Doppler broadening of the hyperfine lines is then given by [27]:

$$
\frac{\Delta \nu}{\nu}=\frac{a}{c t_{d}}
$$

where it appears clearly that it depends not on the distance between the graphite and the activation volume but only on the diffusion delay. This means that for $a \simeq 1 \mathrm{~mm}$, a wavelength of $\lambda=351.5 \mathrm{~nm}$ and $t_{d}=12 \mu \mathrm{s}$ which were our measurement conditions, a resolution of $\Delta \nu \simeq 240$ $\mathrm{MHz}$ was the best to be expected. In the present work we managed to obtain a resolution between 500 and 700 $\mathrm{MHz}$. The broadening could have been reduced by decreasing $a$ and/or increasing $t_{d}$, but obviously at the expense of the efficiency. The ionization volume decreases as $a$. The consequence of the variation of $t_{d}$ on the efficiency is less trivial: it depends of the shape and velocity distribution of the desorbed atomic cloud as we shall see now.
Influence of the desorption geometry on the efficiency The Nd:YAG beam impact zone can be considered as a thermal source of atoms at a temperature $T_{s}$, taken approximately as the maximum surface temperature obtained during the Nd:YAG pulse calculated above. We assume a Maxwell distribution for the velocity of the desorbed atoms, corrected by a $\cos i$ factor (the angle $\widehat{i}$ is defined in Fig. 9 with $0 \leq i \leq \frac{\pi}{2}$ ) representing an assumed enhanced probability for forward angles [27]. Then the mean number of atoms per unit of volume having their velocity vector between $\mathbf{v}$ and $\mathbf{v}+\mathrm{d} \mathbf{v}$ in the case of a $z$-axis rotation symmetric distribution is :

$$
f(\mathbf{v}) \mathrm{d}^{3} \mathbf{v} \equiv C \cos i \exp \left(-\frac{1}{2} \frac{m v^{2}}{k_{B} T_{s}}\right) \mathrm{d}^{3} \mathbf{v}
$$

where $v \equiv|\mathbf{v}|$. By replacing $\mathbf{v}$ by $\frac{\mathbf{r}}{t_{d}}$ and taking advantage of the rotation symmetry around the $z$-axis one has :

$$
\begin{aligned}
& f(r, \cos i) \mathrm{d} r \mathrm{~d} \cos i= \\
& 2 \pi \mathcal{C} r^{2} \cos i \exp \left(-\frac{1}{2 k_{B} T_{s}} \frac{m r^{2}}{t_{d}^{2}}\right) \mathrm{d} r \mathrm{~d} \cos i
\end{aligned}
$$

where $r \equiv|\mathbf{r}|$. The normalization constant is :

$$
\mathcal{C}=4\left(\frac{3}{2 \pi}\right)^{\frac{3}{2}} \frac{1}{t_{d}^{3} v_{r m s}^{3}} N
$$

where $N$ is the total number of desorbed atoms and $v_{r m s}$ is the square root of the mean square velocity $v_{r m s} \equiv\left(\bar{v}^{2}\right)^{\frac{1}{2}}$. In our case, with atoms of mass $\simeq 190$ and assuming a surface temperature of the order of the value obtained for a Pyr. N., one obtains $v_{r m s} \simeq 720 \mathrm{~m} \cdot \mathrm{s}^{-1}$. By integration of Eq. 3 over the ionization volume, it is easy to calculate the proportion of the desorbed atoms exposed to the three laser beams. This probability is represented as a function of the distance $d$ for four different values of the delay $t_{d}$ in Fig. 10. One can see that for a given $d$ the probability shows a maximum for a given $t_{d}$. For instance if one considers a distance $d=5 \mathrm{~mm}$, then the maximum probability is encountered for a diffusion delay $t_{d}=10 \mu \mathrm{s}$ and is equal to $2 \%$ only (experimental conditions were $t_{d}=12 \mu$ s and $d=4.5 \mathrm{~mm}$ ). Seeing the curves presented in Fig. 10 it is tempting to perform the ionization closer to the graphite surface and the sooner after the desorption. The choice for $d$ is in fact limited by the electrode positions $3 \mathrm{~mm} \leq d \leq 31 \mathrm{~mm}$. Furthermore, from Eq. 2 it is clear that decreasing $t_{d}$ would result in degrading the resolution, in order to recover the resolution it would then be necessary to decrease $a$ hence the ionization volume and consequently the efficiency. All this shows that our experimental conditions result, as indicated above, from a compromise between resolution and efficiency.

Estimated efficiency The various contributions to the overall efficiency are listed in Tab. 2. A HFS frequency is selected for the measurement and the corresponding transition rate is taken into account in the efficiency evaluation; 


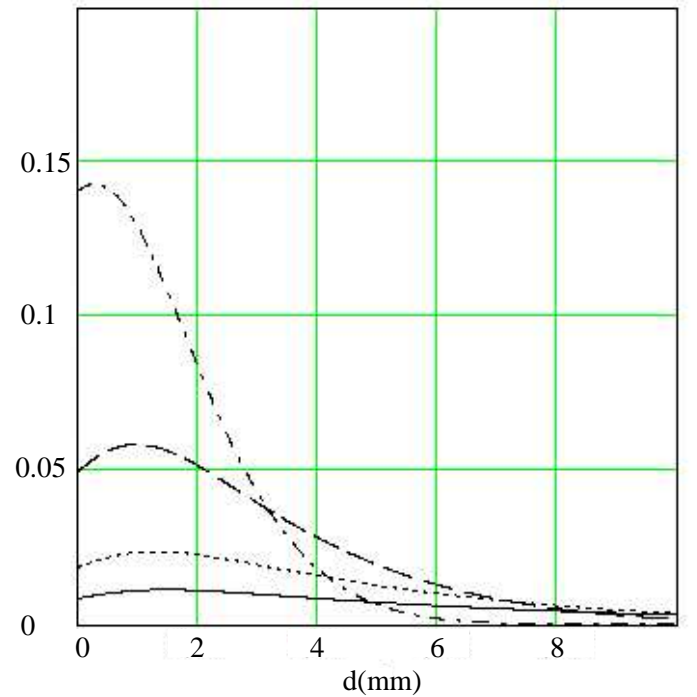

Fig. 10. Probability for one desorbed atom to be inside the ionization parallelepiped as a function of the distance $d$ between the graphite surface and the three laser beam overlap region after a diffusion delay of $t_{d}=5 \mu$ s (dot-dashed line), $t_{d}=10 \mu \mathrm{s}$ (dashed line), $t_{d}=15 \mu \mathrm{s}$ (dotted line) and $t_{d}=20 \mu \mathrm{s}$ (solid line).

Table 2. Overall efficiency of the COMPLIS system.

\begin{tabular}{ll}
\hline Measured efficiency & $10^{-6}$ \\
\hline Proportion of desorbed atoms & 0.5 \\
Ionization volume & 0.02 \\
Ionization process & 0.1 \\
COMPLIS line transmission & 0.5 \\
MCP detection efficiency & 0.6 \\
\hline Estimated efficiency & $3 \cdot 10^{-4}$ \\
\hline Remaining factor & 300 \\
\hline
\end{tabular}

- The calculation of the proportion of desorbed atoms was made by assuming a shallow gaussian distribution of the atoms in the few first tens of $\AA$ in the graphite substrate;

- The effect of the geometrical configuration of the ionization process has been determined as just described;

- The efficiency of the ionization process has been estimated in the context of the very similar RIS spectrometer device PILIS [28, 29];

- The value of the MCP detection efficiency is taken from ref. [30].

Yet, the remaining factor is very large. One part of it comes from the thermal population of metastable atomic states during the desorption, these excited atoms are then lost for the resonance ionization process. Despite the fact that the surface-heating power is limited thanks to a careful deceleration of the beam, the population of metastable states seems to constitute an important factor of loss, impossible to control. The rest can originate from various conjugate processes: a bad vertical overlap between the implantation zone and the desorption focus segment, an

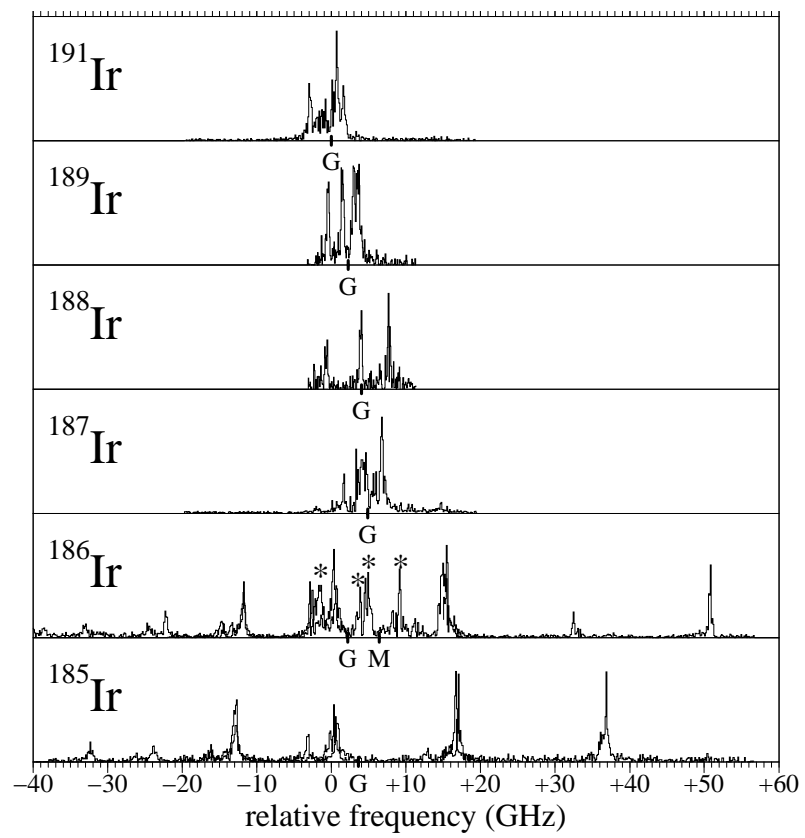

Fig. 11. Hyperfine spectra for some of the isotopes observed during this experiment. For mass 186, a $*$ symbol indicates the peaks which are attributed to the isomeric state. The center of gravity positions of the various HFS are indicated by a "G" symbol (and a "M" symbol for ${ }^{186} \mathrm{Ir}^{m}$ ) in order to underline the isotope shift.

overestimated overlap between the different laser beams and an overestimated overlap between the ionization region and the pulsed atomic cloud. A significant part of the desorbed products may be ionized directly during the desorption process. Results rather similar have been reported in ref. [6]: the theoretical overall efficiency has also been estimated to be around $10^{-4}$ and the measured values obtained with stable samples $\left(10^{-5}\right.$ for gold and $3.10^{-6}$ for platinum) show a large missing factor too.

\section{Analysis of the experimental data}

\subsection{Hyperfine spectra}

The hyperfine spectra in the ${ }^{4} F_{\frac{9}{2}} \rightarrow{ }^{6} F_{\frac{11}{2}}$ transition at $351.5 \mathrm{~nm}$ have been recorded for the iridium isotopes ${ }^{191,193} \mathrm{Ir}$ (stables), ${ }^{182} \mathrm{Ir}$ to ${ }^{189} \mathrm{Ir}$ and for the isomeric state of ${ }^{186} \mathrm{Ir}$. Part of the spectra obtained during this experiment are shown in Fig. 11. The $A=186$ case happens to be the most special one and deserves a few comments. Firstly the two long lived states show rather similar halflives $\left(T_{\frac{1}{2}}=15.8 \mathrm{~h}\right.$ for ${ }^{186} \mathrm{Ir}^{g}$ and $T_{\frac{1}{2}}=1.6 \mathrm{~h}$ for $\left.{ }^{186} \mathrm{Ir}^{m}\right)$ and the two superimposed HFS had to be carefully disentangled. Secondly, the HFS of the ground state spans more than $100 \mathrm{GHz}$ and exhibits a very large number of peaks due to the high values for both the nuclear and atomic spins. Since the maximum range of the monomode cavity is limited to $30 \mathrm{GHz}$, the effective doubled-frequency scan 


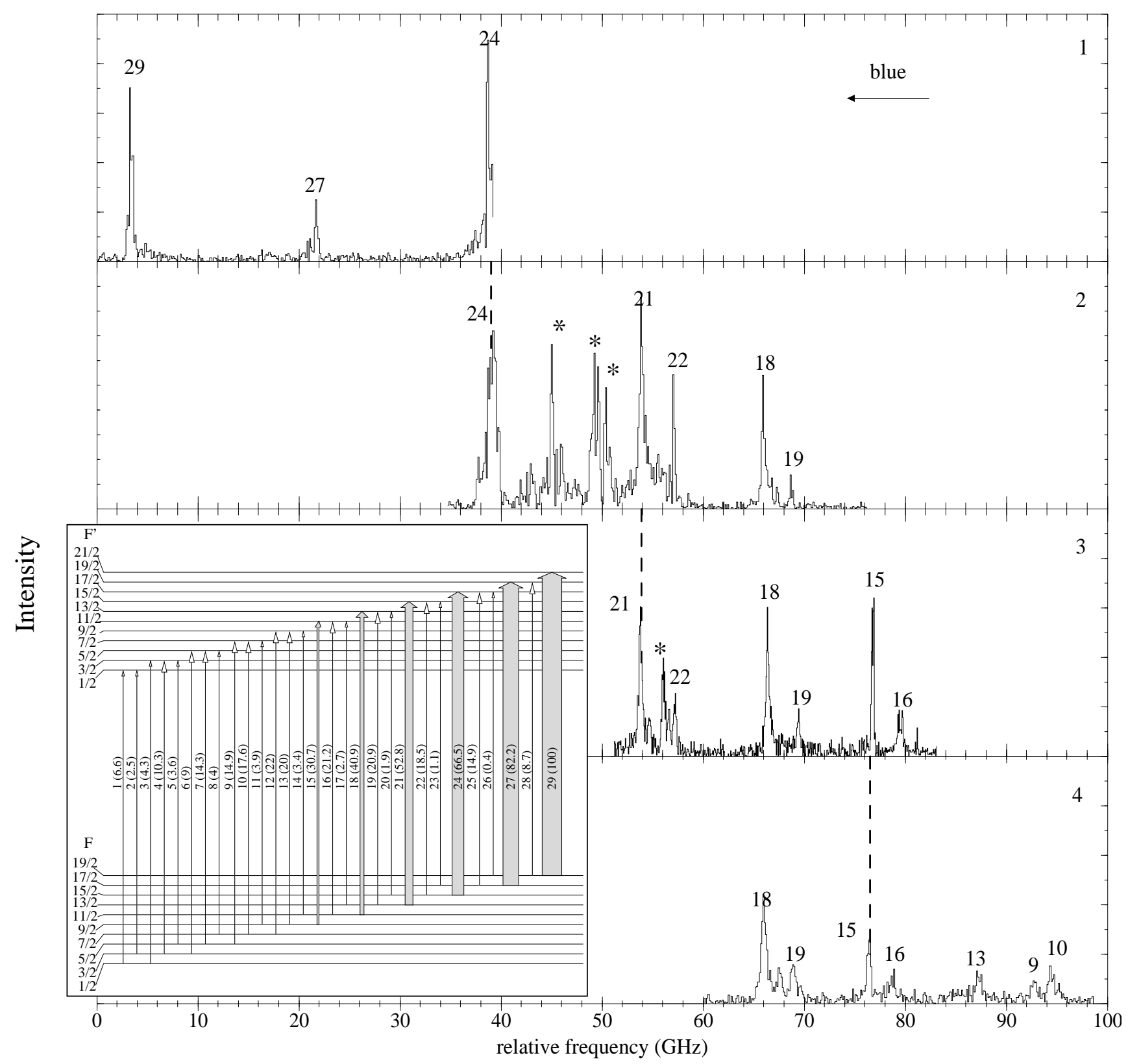

Fig. 12. Partial recordings of the hyperfine spectra of ${ }^{186} \operatorname{Ir}^{g}$ and ${ }^{186} \operatorname{Ir}^{m}$. The common peaks used in order to link the different parts are indicated by doted lines. Each peak is identified by the labels of the hyperfine transitions displayed in the insert (the relative intensities are indicated between parentheses). Peaks belonging to the isomeric state are indicated with $*$-symbols.

of the HFS is limited to $60 \mathrm{GHz}$. The various parts of the spectrum were then linked by superposition of common peaks as shown in Fig. 12. This procedure obviously leads to a supplementary source of uncertainties in the determination of both the hyperfine constants and the position of the HFS center of gravity. As can be seen by comparing in Fig. 12 the insert and the experimental spectra, the relative intensities of the peaks are far from being in agreement with the expected relative intensities of the transitions. This high variability of the ion signal probably originates from inhomogeneities in the implanted population and/or the quality of the graphite surface as it moves under the Nd:YAG laser focus segment. Therefore each section of the spectrum was recorded several times.

\subsection{Magnetic dipole moments}

The magnetic hyperfine interaction constants $A$ have been extracted from the recorded spectra; their values are reported in table 3 . Along with those results are also reported the values of the magnetic dipole moments $\mu_{I}$ extracted from the hyperfine constants of the ground state ${ }^{4} F_{\frac{9}{2}}$. The value for the electronic shell magnetic field at 
Table 3. Experimental data on dipole hyperfine constants for the two atomic states ${ }^{4} F_{\frac{9}{2}}$ and ${ }^{6} F_{\frac{11}{2}}$, magnetic moments extracted from the ${ }^{4} F_{\frac{9}{2}}$ data (see text) and values available in literature. The uncertainties were calculated by taking the peak positions from each recording of the same spectrum (see text). Each measurement is considered as independent from the others, then one set of frequency is obtained for each peak and was treated as a random sample.

\begin{tabular}{|c|c|c|c|c|c|c|}
\hline \multirow[t]{2}{*}{ mass } & \multirow[t]{2}{*}{$\mathrm{I}^{\pi}$} & \multicolumn{2}{|l|}{$\mathrm{A}(\mathrm{GHz})$} & \multicolumn{3}{|c|}{$\mu\left(\mu_{N}\right)$} \\
\hline & & ${ }^{4} F_{\frac{9}{2}}$ & ${ }^{6} F_{\frac{11}{2}}$ & exp. & lit. & ref. \\
\hline \multirow[t]{2}{*}{193} & $\frac{3}{2}^{+}$ & $+0.06265537(2)[31]$ & & & & \\
\hline & & $+0.064(3)$ & $+0.308(4)$ & $+0.168(8)$ & $+0.1636(6)$ & {$[31]$} \\
\hline \multirow[t]{2}{*}{191} & $\frac{3}{2}^{+}$ & $+0.05752128(3)[31]$ & & & & \\
\hline & & $+0.058(1)$ & $+0.288(3)$ & $+0.152(4)$ & $+0.1507(6)$ & [31] \\
\hline 189 & $\frac{3}{2}^{+}$ & $+0.056(2)$ & $+0.267(3)$ & $+0.147(7)$ & $0.13(+8)$ & {$[32]$} \\
\hline 188 & $1^{-}$ & $+0.187(6)$ & $+0.828(8)$ & $+0.33(1)$ & $0.302(10)$ & {$[33]$} \\
\hline 187 & $\frac{3}{2}^{+}$ & $+0.065(3)$ & $+0.323(5)$ & $+0.17(1)$ & & \\
\hline $186^{g}$ & $\stackrel{2}{5}^{+}$ & $+0.43(2)$ & $+2.06(2)$ & $+3.8(2)$ & $3.88(5)$ & {$[32]$} \\
\hline $186^{m}$ & $2^{-}$ & $-0.189(8)$ & $-0.86(1)$ & $-0.66(3)$ & $0.638(8)$ & {$[34]$} \\
\hline 185 & $\frac{5}{2}^{-}$ & $+0.594(13)$ & $+2.83(1)$ & $+2.59(7)$ & $2.605(13)$ & {$[35]$} \\
\hline 184 & $5^{-}$ & $+0.082(3)$ & $+0.374(4)$ & $+0.72(3)$ & $0.695(5)$ & {$[35]$} \\
\hline 183 & $\frac{5}{2}^{-}$ & $+0.55(2)$ & $+2.593(9)$ & $+2.40(8)$ & & \\
\hline 182 & $3^{+}$ & $+0.49(3)$ & $+2.49(2)$ & $+2.6(2)$ & $2.28(8)$ & {$[36]$} \\
\hline
\end{tabular}

Table 4. Experimental data on quadrupole hyperfine constants for the two atomic states ${ }^{4} F_{\frac{9}{2}}$ and ${ }^{6} F_{\frac{11}{2}}$; averaged values of spectroscopic quadrupole moments extracted from the ${ }^{4} F_{\frac{9}{2}}$ and ${ }^{4} F_{\frac{11}{2}}$ data (see text) and values available in literature.

\begin{tabular}{|c|c|c|c|c|c|c|}
\hline \multirow[t]{2}{*}{ mass } & \multirow[t]{2}{*}{$\mathrm{I}^{\pi}$} & \multicolumn{2}{|l|}{$\mathrm{B}(\mathrm{GHz})$} & \multicolumn{3}{|c|}{$Q_{s}(\mathrm{eb})$} \\
\hline & & ${ }^{4} F_{\frac{9}{2}}$ & ${ }^{6} F_{\frac{11}{2}}$ & exp. & lit. & ref. \\
\hline \multirow[t]{2}{*}{193} & $\frac{3}{2}^{+}$ & $+0.4262355(2)[31]$ & & & & \\
\hline & & $+0.44(3)$ & $-0.75(3)$ & $+0.73(7)$ & $+0.751(9)$ & {$[40]$} \\
\hline \multirow[t]{2}{*}{191} & $\frac{3}{2}^{+}$ & $+0.4712045(3)[31]$ & & & & \\
\hline & & $+0.48(2)$ & $-0.87(4)$ & $+0.82(8)$ & $+0.816(9)$ & {$[40]$} \\
\hline 189 & $\frac{3}{2}^{+}$ & $+0.49(1)$ & $-0.91(2)$ & $+0.85(7)$ & $+0.878(10)$ & {$[4]$} \\
\hline 188 & $1^{-}$ & $+0.27(2)$ & $-0.48(3)$ & $+0.46(5)$ & $+0.484(6)$ & [4] \\
\hline 187 & $\frac{3}{2}^{+}$ & $+0.50(2)$ & $-0.91(6)$ & $+0.9(1)$ & $+0.941(11)$ & {$[4]$} \\
\hline $186^{g}$ & $5^{+}$ & $-1.5(5)$ & $+2.7(9)$ & $-2.6(9)$ & $-2.548(31)$ & {$[4]$} \\
\hline $186^{m}$ & $2^{-}$ & $+0.90(7)$ & $-1.51(8)$ & $+1.5(2)$ & $+1.456(17)$ & {$[4]$} \\
\hline 185 & $\frac{5}{2}^{-}$ & $-1.0(3)$ & $+1.8(2)$ & $-1.7(6)$ & $-2.06(14)$ & [35] \\
\hline 184 & $5^{-}$ & $+1.3(2)$ & $-3.0(2)$ & $+2.5(4)$ & $+2.1(3)$ & {$[43,44]$} \\
\hline 183 & $\frac{5}{2}^{-}$ & $-1.1(4)$ & $+1.9(6)$ & $-1.8(7)$ & & \\
\hline 182 & $3^{+}$ & $-1.1(4)$ & $+1.5(5)$ & $-1.7(6)$ & & \\
\hline
\end{tabular}

the nucleus $\overline{H(0)}$ used in this procedure has been determined from the expression :

$$
A=\frac{\mu_{I}}{I} \frac{\overline{H(0)}}{J}
$$

(where $I$ and $J$ stand for the total angular momenta of the nuclear and atomic state respectively), using the very precise values of $A$ and magnetic moment for ${ }^{191} \mathrm{Ir}$ from [31] which are also reported in Tab. 3. This means that no correction for the hyperfine anomaly has been taken into account. This is justified by the size of our relative uncertainties which range from $2.5 \%$ in the best case namely the stable ${ }^{191}$ Ir to $7.5 \%$. S. Büttgenbach et al. report a value for the differential hyperfine anomaly of ${ }^{191} \Delta_{\text {exp }}^{193}=$ $-0.023(10) \%[13]$ for the atomic ground state determined from the ratio $\mu_{I}\left({ }^{193} \operatorname{Ir}\right) / \mu_{I}\left({ }^{191} \operatorname{Ir}\right)=1.0890(1)[37]$. This very small value which is explained by the presence of a very pure configuration of two paired $s$-electrons in the ground state as mentioned in section 1 is far beyond the accuracy of our determination of the magnetic moments. On the other hand the value of the differential hyperfine anomaly is believed to be important in the excited levels corresponding to a very pure configuration containing one unpaired $s$-electron plus one unpaired $p$-electron. Büttgenbach et al. propose a value for the differential hyperfine anomaly for a pure $s$-electron configuration ${ }^{191} \Delta_{s}^{193}=$ $0.64(7) \%$ [13] which happens to be in good agreement with the value obtainable from the Moskowitz and Lombardi empirical rule [38]. Anyway, the exact contribution of the contact interaction of the unpaired $s$-electron to the hyperfine splitting $\mathrm{a}_{s}$ in the $5 d^{7} 6 s 6 p$ configuration of our ${ }^{4} F_{\frac{11}{2}}$ excited level is unknown and a supplementary con- 
tribution should arise from the presence of a $p_{\frac{1}{2}}$-electron. Besides, the Moskowitz-Lombardi rule was established in the case of $s$-electrons and nuclear spin $I=l \pm \frac{1}{2}$. It may be quite hazardous to apply it directly to some cases we are concerned with, like ${ }^{185} \mathrm{Ir}$ where, as we shall see latter, the nuclear ground state is mainly of the $h_{\frac{9}{2}}$ spherical shell nature and yet has a spin $I=5 / 2$. For all those reasons we used only the hyperfine constants of the ${ }^{4} F_{\frac{9}{2}}$ levels for the determination of the $\mu_{I}$ values.

\subsection{Spectroscopic quadrupole moments}

The spectroscopic quadrupole moments $Q_{s}$ listed in Tab. 4 were extracted from the $B$ hyperfine electric constants :

$$
B=e Q_{s} \overline{\varphi_{J J}(0)}
$$

where, using the usual notation, $\overline{\varphi_{J J}(0)}$ is the vector gradient of the electric field of the orbital electrons at the nucleus. Though it was not precisely calculated in the case of iridium the Sternheimer effect is believed to be important. Murakawa and Kamei have determined experimentally a very important anti-shielding coefficient due to the $5 d$ electronic orbital $R_{5 d} \simeq-0.4$ in ${ }^{139} \mathrm{La},{ }^{175} \mathrm{Lu}$ and ${ }^{201} \mathrm{Hg}$ [39] which was confirmed by the calculation [41]. Tanaka et al. report a value of $R_{5 d}=-0.35(28)$ for ${ }^{191} \mathrm{Ir}$ and $R_{5 d}=-0.32(28)$ for ${ }^{193} \mathrm{Ir}$ by comparing the quadrupole moments from electronic-atom experiments to the very precise values they obtained by studying the hyperfine structure of muonic $M$ x rays [42]. It turns out from their results that the Sternheimer effect should stay constant for the different isotopes of the same element. Their value of $Q_{s}=+0.816(9)$ eb (corrected for Sternheimer effect) for ${ }^{191} \mathrm{Ir}$ was used here along with the $B=+0.4712045(3)$ $\mathrm{GHz}$ value of ref. [31] for the ${ }^{4} F_{\frac{9}{2}}$ state and our value of $B=-0.87(4) \mathrm{GHz}$ for the ${ }^{6} F_{\frac{11}{2}}$ state in order to determine the $Q_{s}$ values. We have chosen then to report in Tab. 4 the mean values between the determinations coming from the two different atomic states.

\subsection{Isotope shift}

The usual procedure is undertaken in order to extract the mean square charge radius variations $\delta\left\langle r^{2}\right\rangle$ from the measured IS. The shifts of the various HFS centers of gravity measured from the one of the stable isotope ${ }^{191} \mathrm{Ir}$ are given in Tab. 5. The observed IS shift is the sum of a mass shift (MS) and a volume (field) shift (FS):

$$
\Delta \nu_{I S}=\Delta \nu_{M S}+\Delta \nu_{F S}
$$

The MS which is composed of the normal mass shift (NMS) and the specific mass shift (SMS) has been estimated as:

$$
\Delta \nu_{M S}=\mathcal{C} \frac{A-A_{\text {ref }}}{A A_{\text {ref }}}
$$

where

$$
\mathcal{C}=\mathcal{C}_{N M S}+\mathcal{C}_{S M S}=\frac{1 \pm 0.5}{1836.1} \nu_{0}
$$

The contribution of the SMS has been taken as the semiempirical determination of Heilig and Steudel for a pure transition of the $s^{2} \rightarrow s p$ type [45]:

$$
\Delta \nu_{S M S}=(0 \pm 0.5) \Delta \nu_{N M S}
$$

The frequency value used for the fine structure transition $\nu_{0}$ is $852979.0949 \mathrm{GHz}$ [46]. The FS is written as the product:

$$
\Delta \nu_{F S}=F \lambda
$$

where $F$ is the atomic factor which contains the variation of the electronic wave function at the nucleus during the transition and is defined as [47]:

$$
F=\frac{2 \pi}{3} \frac{Z e^{2}}{h} \Delta|\Psi(0)|^{2}
$$

and $\lambda$ which is usually called the nuclear parameter though it contains some atomic information. Following the definition of the electronic factors $C_{i}$ of Seltzer [48]:

$$
\Delta \nu_{F S}=F \lambda=C_{1}\left(\delta\left\langle r^{2}\right\rangle+\frac{C_{2}}{C_{1}} \delta\left\langle r^{4}\right\rangle+\frac{C_{3}}{C_{1}} \delta\left\langle r^{6}\right\rangle \ldots\right)
$$

where the last term in parenthesis is taken for $\lambda$ and contains obviously the ratios of the electronic factors $C_{i}$. In order to get good results the electronic charge density at the nucleus was calculated with the newest version of the $a b$ initio fully relativistic Multiconfiguration Dirac Fock Code [49] where the nuclear charge distribution was assumed to have a spherical Fermi-type shape. A similar approach has been adopted in the previous works on IS in this region namely mercury [2], gold [50] and platinum [9]. This leads to

$$
4 \pi \Delta|\Psi(0)|^{2}=-1026.24 a_{0}^{-3}
$$

and by using the expression of the $F$ factor:

$$
F=\frac{2 \pi^{2}}{3} c \frac{\alpha^{4}}{\lambda_{c}^{3}} Z 4 \pi a_{0}^{3} \Delta|\Psi(0)|^{2}\left[\mathrm{GHz} \cdot \mathrm{fm}^{-2}\right]
$$

we finally get:

$$
F_{351}=-30.94 \mathrm{GHz} \cdot \mathrm{fm}^{-2}
$$

The nuclear parameter values from the mass 191 reference:

$$
\lambda^{191-A}=\frac{1}{F_{351}}\left(\Delta \nu_{I S}^{191-A}-\Delta \nu_{M S}^{191-A}\right)
$$

are reported in Tab.5. The nuclear parameters $\lambda$ are treated using the two-parameter model where the global change in the mean square charge radius is considered as resulting from the cumulated effects of the change in the spherical volume and in the shape:

$$
\delta\left\langle r^{2}\right\rangle=\delta\left\langle r^{2}\right\rangle_{s p h}+\delta\left\langle r^{2}\right\rangle_{d e f}
$$

A uniform nuclear charge distribution within a purely axial shape is assumed so that the second order radial moment of the charge distribution results in [51]:

$$
\delta\left\langle r^{2}\right\rangle=\delta\left\langle r^{2}\right\rangle_{s p h}+\frac{5}{4 \pi}\left\langle r^{2}\right\rangle_{s p h} \delta \beta_{2}^{2}
$$


Table 5. Results from the Ir IS in the ${ }^{4} F_{9 / 2} \rightarrow{ }^{6} F_{11 / 2} 351.5 \mathrm{~nm}$ line. $\lambda$ is the nuclear parameter obtained from Eq. $5, \delta\left\langle r^{2}\right\rangle$ and $\delta\left(\beta_{2}^{2}\right)$ are the solutions of the two-equation system Eq. 6 and Eq. 7. The uncertainties in the absolute value of the axial deformation parameter $\left\langle\beta_{2}^{2}\right\rangle^{1 / 2}$ originating from the errors in the determination of the reference value taken for ${ }^{191}$ Ir are not taken into account here. The choice of this value is discussed in the text.

\begin{tabular}{llllll}
\hline mass & $\Delta \nu^{191-A}$ & $\lambda^{191-A}$ & $\delta\left\langle r^{2}\right\rangle^{191-A}$ & $\delta\left(\beta_{2}^{2}\right)$ & $\left\langle\beta_{2}^{2}\right\rangle^{1 / 2}$ \\
& $\mathrm{GHz}$ & & & & \\
\hline 193 & $-2.00(1)$ & $+0.0655(8)$ & $+0.0691(8)$ & $-0.00319(7)$ & $0.144(1)$ \\
191 & 0 & 0 & 0 & 0 & 0.155 \\
189 & $+2.21(5)$ & $-0.072(2)$ & $-0.076(2)$ & $+0.0025(2)$ & $0.163(1)$ \\
188 & $+4.03(8)$ & $-0.132(3)$ & $-0.140(4)$ & $+0.0016(3)$ & $0.160(1)$ \\
187 & $+4.85(6)$ & $-0.158(3)$ & $-0.168(3)$ & $+0.0038(3)$ & $0.167(1)$ \\
& & & & & \\
$186^{g}$ & $+2.16(7)$ & $-0.072(3)$ & $-0.073(4)$ & $+0.0166(3)$ & $0.202(1)$ \\
$186^{m}$ & $+6.38(3)$ & $-0.208(2)$ & $-0.221(2)$ & $+0.0037(2)$ & $0.167(1)$ \\
185 & $+3.60(5)$ & $-0.119(3)$ & $-0.123(3)$ & $+0.0168(3)$ & $0.202(1)$ \\
184 & $+5.12(3)$ & $-0.168(2)$ & $-0.176(3)$ & $+0.0168(2)$ & $0.202(1)$ \\
183 & $+5.93(7)$ & $-0.195(4)$ & $-0.203(4)$ & $+0.0190(4)$ & $0.207(1)$ \\
182 & $+8.2(1)$ & $-0.269(6)$ & $-0.283(6)$ & $+0.0167(5)$ & $0.202(1)$ \\
\hline
\end{tabular}

In order to take into account the influence of the higher order terms of Eq. 4 the nuclear parameter is re-written integrating two correction parameters $x$ and $y$ as [51]:

$$
\lambda=(1+x) \delta\left\langle r^{2}\right\rangle_{s p h}+(1+y) \frac{5}{4 \pi}\left\langle r^{2}\right\rangle_{s p h} \delta \beta_{2}^{2}
$$

with

$$
\begin{aligned}
& x=\frac{C_{2}}{C_{1}} \frac{10}{7} R^{2}+\frac{C_{3}}{C_{1}} \frac{5}{3} R^{4} \\
& y=\frac{C_{2}}{C_{1}} 2 R^{2}+\frac{C_{3}}{C_{1}} 3 R^{4}
\end{aligned}
$$

where $R$ is the nuclear radius taken as $R=r_{0} \bar{A}^{1 / 3}$. Recent values for the $C_{i}$ ratios were calculated by Torbhom et $a l$. in the framework of the multiconfiguration Dirac-Fock method for the II $a$ and II $b$ group elements [47]. For Ir it is necessary to take interpolated values:

$$
\frac{C_{2}}{C_{1}}=-1.12 \cdot 10^{-3} \mathrm{fm}^{2} \quad \frac{C_{3}}{C_{1}}=+3.45 \cdot 10^{-6} \mathrm{fm}^{4}
$$

which are the ones we used here. We give for comparison the values of Seltzer [48]: $C_{2} / C_{1}=-1.04 \cdot 10^{-3} \mathrm{fm}^{2}$ and $C_{3} / C_{1}=+2.80 \cdot 10^{-6} \mathrm{fm}^{4}$. The variation of the spherical charge radius $\left\langle r^{2}\right\rangle_{s p h}$ was calculated with the Droplet Model [52] using the revised set of parameters of Myers and Schmidt [53]. The values of the quantities $\delta\left\langle r^{2}\right\rangle$ and $\delta \beta_{2}^{2}$ are then obtained as the solution of the two-parameter procedure from eqs. 6 and 7, they are reported in Tab. 5. The absolute values of the axial deformation parameters $\left|\beta_{2}\right|$ can be determined provided a value of $\beta_{2}$ is known for at least one reference isotope. We have used as a reference the result from Möller and Nix [54] :

$$
\beta_{2}(191)=+0.155
$$

This choice originates from the fact that this number is very close to the average value between $\beta_{2}=+0.140$ which comes from the intrinsic quadrupole moment $Q_{0}$ determined from the measured $Q_{s}$ assuming $K=3 / 2$ for the projection of the nuclear angular momentum $\mathbf{I}$ on the symmetry axis (see next section); and $\beta_{2}=+0.176$ which is obtained at the equilibrium in our $\mathrm{HF}+\mathrm{BCS}$ calculation using a ${ }^{190}$ Os core (see next section). The resulting absolute values of $\beta_{2}$ are reported in Tab. 5. Fig. 13 summarizes the situation. The variations of the charge radius $\delta\left\langle r^{2}\right\rangle$ are represented with respect to the stable reference isotope 191. The iso-deformation curves calculated using the Droplet Model are drawn in Fig. 13 to underline the relative importance of the spherical and deformed contributions to the global variation. An important feature emerges from this graphic which is the sudden rising of the deformation between two mass regions on either side of $A=186$. On the heavier side the deformation increases slowly from $\beta_{2} \simeq 0.14$ to 0.17 from mass 193 to 187 as the mean square charge radius diminishes following a rather regular slope. The isomeric state of ${ }^{186} \mathrm{Ir}$ lies approximately on the continuation of this slope. From mass 187 to mass 186 (ground state) the deformation suddenly increases to $\beta_{2} \simeq 0.2$ and nearly keeps hanging on the iso-deformation line around 0.2 until mass 182 . The parallelism between the experimental curve and Droplet Model curve for $\beta_{2}=0.2$ is striking and clearly shows that from ${ }^{186} \mathrm{Ir}^{g}$ to ${ }^{182} \mathrm{Ir}$ the reduction of the neutron number results only in a variation of the spherical part of the bulk charge distribution. At last, our results are compared with $\delta\left\langle r^{2}\right\rangle$ results previously obtained in this mass-region in Fig. 14.

\section{Discussion}

We discuss the various data obtained on the odd and doubly odd iridium nuclei during this experiment in the framework of a model of an axially symmetric rotor plus one or two quasiparticles (qp). This model has already proved to be able to describe features in the energy spec- 


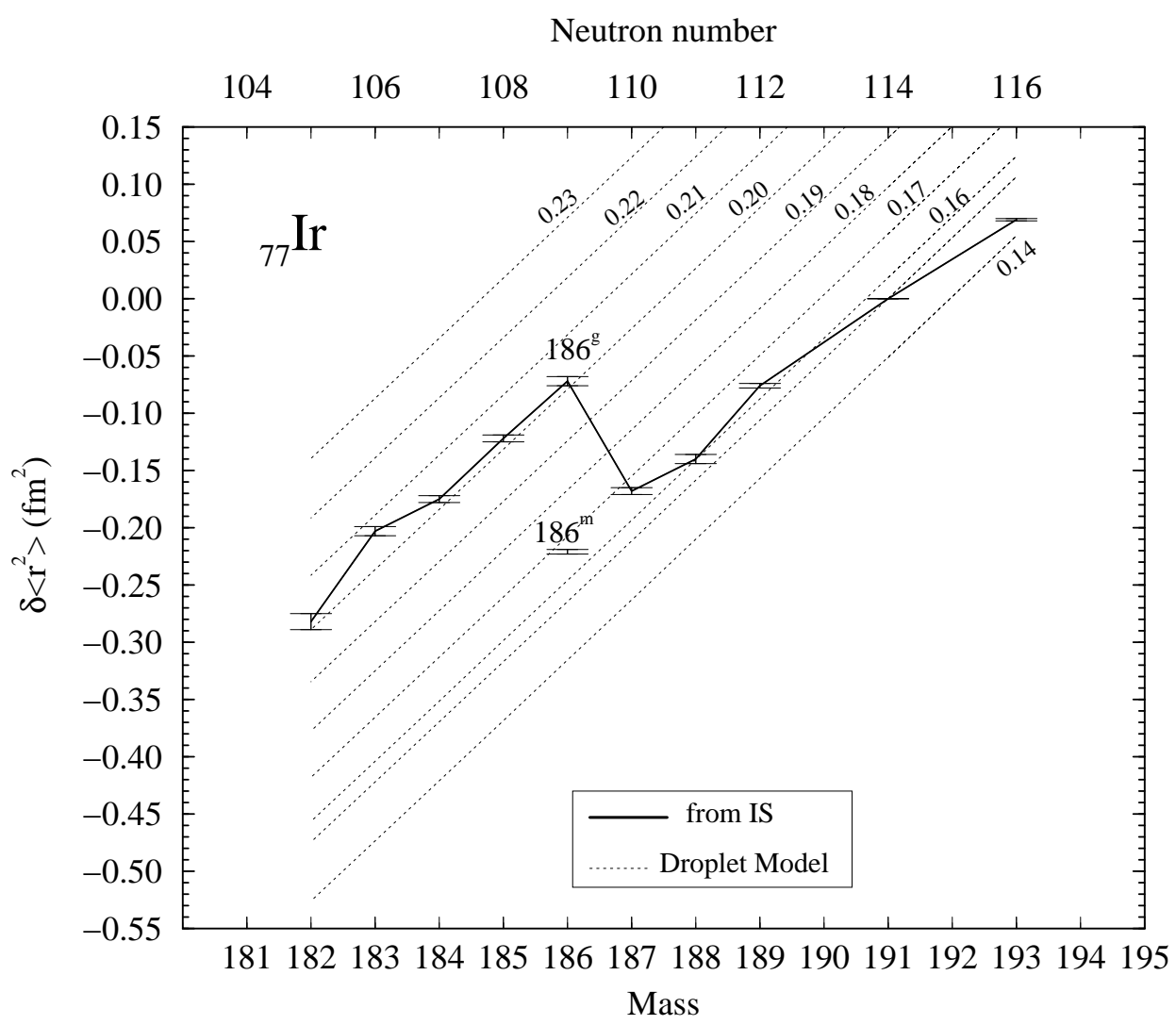

Fig. 13. Mean square charge radius variation of the Ir isotopic series obtained from the IS measurement. ${ }^{191}$ Ir is used as the reference, with $\beta_{2}=0.155$. Iso-deformation curves (see text) are indicated by dotted lines.

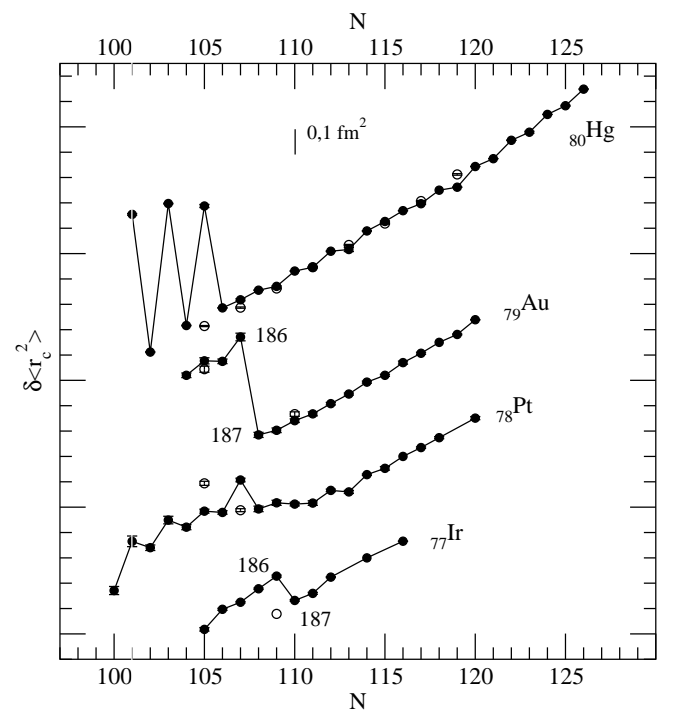

Fig. 14. Mean square charge radius variation obtained from the IS measurement for different isotopic series in the Pt region. Isomeric states are represented by open circles. tra of various nuclei both at low and high spins [55-60]. The number of successful results which have already been obtained using this approach in the transitional Os-Pt-Hg region $[12,59,61,62]$ constitute a strong incentive to use it again in the case of the iridium nuclei.

\subsection{The rotor +1 or 2 qp approach}

\subsubsection{Quasi-particle states}

The single particle (sp) wave functions are calculated using the Constrained Hartree-Fock (CHF) method [55], the nuclear mean field being obtained in a self-consistent way with the effective nucleon-nucleon interaction of the Skyrme type using the SIII set of parameters [63]. The calculations are limited to the case of an axially and time-reversal symmetric mean field. In order to explore the dependence of the sp energies upon axial deformation, the core is constrained in the CHF procedure to have a definite value for the intrinsic (mass) quadrupole moment $Q_{20}$. The pairing correlations are treated using the BCS approach and assuming constant pairing strengths $G_{0 p}$ and $G_{0 n}$ for the protons and the neutrons respectively. The HF wave functions are expanded on a deformed harmonic oscillator basis (see [55]) limited to 13 major shells. The effect of the truncation of the basis is limited by optimizing for each 
nucleus and each deformation the values of the oscillator parameters $\mathrm{q}=\omega_{\perp} / \omega_{z}$ and $\mathrm{b}=\sqrt{m \omega / \hbar}$ with $\omega^{3}=\omega_{z} \omega_{\perp}^{2}$. The CHF calculations are performed for the even-even cores; then the odd nuclear states are described by the one-qp states built on a BCS vacuum wave function of that underlying core noted $|B C S\rangle[56]$ :

$$
\left|\chi_{q}\right\rangle=\eta_{q}^{+}|B C S\rangle
$$

for a given charge state $q$. The odd-odd nuclear states are described by the two-qp states [60]:

$$
\left|\chi_{n p}\right\rangle=\eta_{n}^{+} \eta_{p}^{+}|B C S\rangle
$$

The expectation values of the particle number operator $\widehat{N}$ for a one-qp state or a two-qp state are given respectively by

$$
\langle\widehat{N}\rangle=A+u_{i}^{2}-v_{i}^{2}
$$

or

$$
\langle\widehat{N}\rangle=A+u_{p}^{2}-v_{p}^{2}+u_{n}^{2}-v_{n}^{2}
$$

where $A=\langle B C S|\widehat{N}| B C S\rangle$ and $u_{i}^{2}$ and $v_{i}^{2}$ stand for the usual BCS occupation coefficients of the given qp state in the core. Thus the one-qp wave function is considered to represent the $A+1$ or $A-1$ nucleus depending on the occupation factor values, the same applying for both protons and neutrons in the case of doubly odd nuclei.

\subsubsection{Coupling of the collective and individual degrees of freedom}

Besides the individual degrees of freedom inherent to the qp description, collective degrees of freedom are implemented which are considered as originating from the core dynamical behavior assumed to be that of a pure axial rotor. This is done by replacing the even-particle vacuum energy part of the total energy of the nucleus by the kinetic energy of an axial rotor (for further details see refs. [56] and [60]):

$$
H_{R}=\frac{\hbar^{2}}{2 \Im} \mathbf{R}^{2}
$$

The total hamiltonian then reads

( $i)$ in the odd case:

$$
H=\lambda_{q} \widehat{N}_{q}+H_{R}+\sum \mathcal{E}_{q p}(i) \eta_{i} \eta_{i}^{+}
$$

for a given charge state $q$ and single qp states $i$; (ii) in the doubly odd case:

$$
\begin{aligned}
H=\sum \lambda_{q} \widehat{N}_{q}+H_{R} & \\
& +\sum \mathcal{E}_{q p}(n) \eta_{n} \eta_{n}^{+}+\sum \mathcal{E}_{q p}(p) \eta_{p} \eta_{p}^{+} \\
& +V_{\text {residual }}
\end{aligned}
$$

for neutron qp states $n$ et proton qp states $p$. $V_{\text {residual }}$ is defined by Eq. 5 in Ref. [60]. The extension of the model to the doubly odd nuclei is due to the work of Bennour et al. [60]. The effective interaction SIII used in the CHF calculations to get the qp wave functions is also used to determine the residual part of the hamiltonian. This approach revealed itself to be successful in describing the three test nuclei ${ }^{160} \mathrm{~Tb},{ }^{174} \mathrm{Lu}$ and ${ }^{238} \mathrm{~Np}$ in ref. [60]. In order to account for the deviation from the strictly rigid rotor case, the value of the inertia parameter is allowed to vary as a function of the core rotational angular momentum $R$. As in ref. [59], the values of $\hbar^{2} / 2 \Im(R)$ were deduced from the experimental core energies taken from the rotational bands of the neighbouring even-even Os nuclei [64]. The Coriolis interaction which appears in $H_{R}$ is non diagonal in the standard basis and leads to the mixing of the $K$ and $K \pm 1$ qp states ( $K$ is the projection of the total angular momentum on the symmetry axis). In the case where $K=1 / 2$ it also connects the two time reversed components of the qp wave function $\chi_{\frac{1}{2}}$ and $\chi_{-\frac{1}{2}}$ leading to a diagonal energy term governed by the intrinsic decoupling parameter :

$$
a_{i n t}=-\left\langle\chi_{\frac{1}{2}}\left|j_{+}\right| \chi_{-\frac{1}{2}}\right\rangle
$$

which is to play a significant role in our following analysis. In any case, the total hamiltonian is diagonalized in the qp basis including all the states within $5 \mathrm{MeV}$ from the Fermi level. For convenience a total nuclear state $\Psi_{M}^{I}=$ $\sum_{K} C_{K}^{I} \psi_{K M}^{I}$ will then be designated by the Nilsson label $\mathrm{I} K^{\pi}\left[N n_{3} \Lambda\right]$ of its main component on the vectors of the standard basis $\psi_{K M}^{I}$ as defined in [56].

For the odd nuclei the full wave function including the Coriolis mixing is used for the calculation of the static moments. Only the collective part of the intrinsic quadrupole moment was taken into account in the calculation of the spectroscopic quadrupole moments. The magnetic moments were calculated following the method of ref. [58] using the approximation $g_{R}=Z / A$ for the value of the effective gfactor for the rotational motion. In the case of the doubly odd nuclei, the calculation for this quantity was restricted to pure $K$ neutron-proton configurations using the expression $[65]$ :

$$
\mu_{I}=\frac{K}{I+1}\left(g_{K p} K_{p}+g_{K n} K_{n}+g_{R} \frac{I^{2}+I-K^{2}}{K}\right)
$$

where $g_{K p}$ and $g_{K n}$ are the g-factors associated with respectively the sp proton and neutron states in the deformed HF field. These quantities are defined in refs. [66, 67] as:

$$
g_{K}=\frac{1}{K}\left(K g_{l}+\left(g_{s}-g_{l}\right)\left\langle\chi_{K}\left|s_{3}\right| \chi_{K}\right\rangle\right)
$$

where $g_{l}$ and $g_{s}$ are the orbital and the spin g-factors respectively. When using this $\mu_{I}$ formula it is important to remember that, in the case where the two angular moment projections on the symmetry axis $K_{p}$ and $K_{n}$ are anti-aligned, the $K \geq 0$ convention leads to:

- place a minus sign before $K_{p}$ when $K_{n}>K_{p}$; - place a minus sign before $K_{n}$ when $K_{p}>K_{n}$.

The calculation of the quantity $\left\langle\chi_{K}\left|s_{3}\right| \chi_{K}\right\rangle$ was performed in the spherical basis using the overlaps $\langle\beta j K \mid \alpha K\rangle$ of the 
Eq. [9] of ref. [56] generated by the CHF program. This means that no Coriolis effects were taken into account in our calculations of the magnetic moment of the oddodd nuclei except the effects due to the diagonal term which are associated with the so-called magnetic decoupling parameter $b_{0}$, defined for instance in ref. [67]. This parameter enters explicitly in the calculation of the magnetic moment of a state $\Psi_{M}^{I}=C_{0}^{I} \psi_{0 M}^{I}+C_{1}^{I} \psi_{1 M}^{I}$ of a pure $K_{p}=K_{n}=1 / 2$ neutron-proton configuration (i.e. no contribution from $I=0$ or $I=1$ states of any other neutron-proton configuration) as can be seen in the Eq. [2] of ref. [68].

\subsubsection{Limitations}

Our approach suffers however from important limitations. The first one is the non-conservation of the particle number, this is a severe restriction in a mass region where polarization effects due the unpaired particle(s) are known to be of importance and especially to play a role in the stabilization of the nuclear shape. Besides it has been shown that a significant improvement in the description of global the properties the odd-nuclei was obtained when the qp states are created by perturbation on a fully selfconsistently determined odd-particle core [69]. The second limitation is the restriction to a static axially symmetric core. Shape mixing is then obviously neglected while it is well known also to be a major issue in this mass region : it has been the object of various detailed theoretical studies among which one can mention the work of Ref. [70] on the light $\mathrm{Hg}$ nuclei. However the configuration mixing problem revealed itself much more involved than expected as was shown in Ref. [71]. In that work, HF+BCS calculations were performed in the approximation of the expectation value method using the same nucleon-nucleon interaction as here (Skyrme SIII) and Bohr dynamics for soft nuclei of different mass regions. For ${ }^{186} \mathrm{Pt}$ in particular, a continuous decrease of the pairing force by only $10 \%$ is seen to by sufficient to induce a displacement of the $0_{1}^{+}$wave function density in the $(\beta, \gamma)$ sextan from the oblate side to the prolate side via complete $\gamma$-softness. More recently (state of the art) Hartree-Fock-Bogoliubov (HFB) calculations were performed by the group of Bruyères-le-Châtel [72] using the D1S Gogny force [73]. The potential energy surfaces (PES) obtained for ${ }^{180,184,186,192}$ Os are shown in Fig. 15. The PES for ${ }^{192}$ Os shows a mexican-hat shaped minimum which is characteristic of a completely $\gamma$-soft nucleus. As neutrons are removed, the energy minimum approaches the prolate edge, at mass 180 the PES is characteristic of a clearly prolate nucleus. This evolution is somewhat expectable from empirical considerations : the well known values of spin and parity of the odd Ir nuclei ground states undergo a sudden change when going away from stability : $3 / 2^{+}$for $A=193$ (stable), 191 (stable), 189 and 187 and then, $5 / 2^{-}$for $A=185$ and 183. It has been shown that the $5 / 2^{-}$ground state of ${ }^{185} \mathrm{Ir}$ can be interpreted as being the band-head of a strongly Coriolis perturbed rotational band built on the $1 / 2^{-}[541]$ Nilsson orbital [59] and the same conclusion can be drawn for the
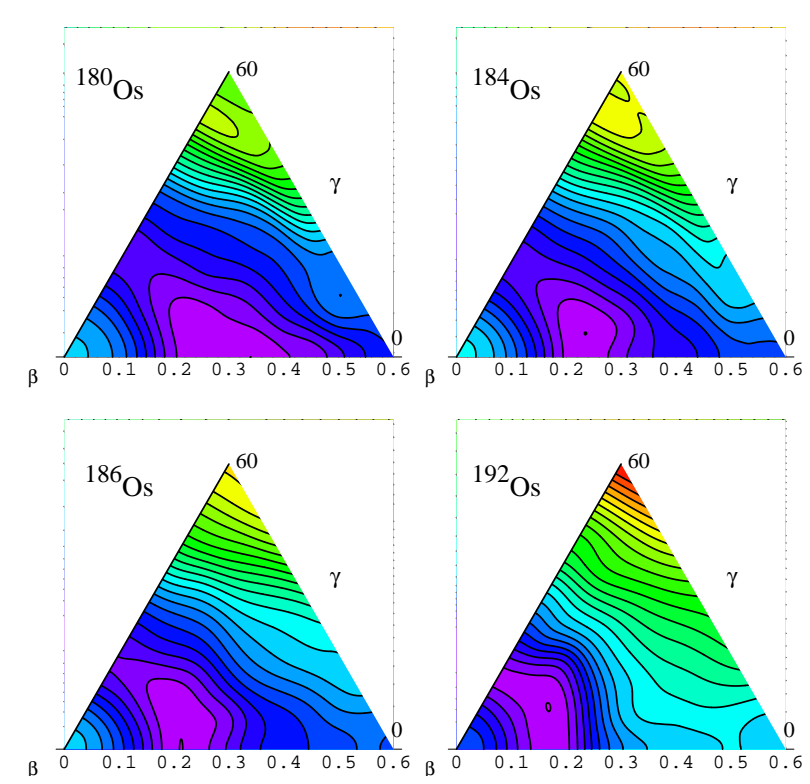

Fig. 15. Potential energy surface $V(\beta, \gamma)$ calculated for $180,184,186,192$ Os obtained in the constrained HFB calculations [72]. Contours are separated by $1 \mathrm{MeV}$.

$5 / 2^{-}$ground state of ${ }^{183} \mathrm{Ir}$ [74]. On the other hand, the $3 / 2^{+}$ground state of $187,189,191,193$ Ir belongs to a positive parity group which is more difficult to represent in static calculations (whether the triaxial degree of freedom is released or not [75]). This is coherent with the general trend of the PES obtained in the constrained HFB calculations shown in Fig. 15 even though no dramatic change is visible between $\mathrm{A}=186$ and $\mathrm{A}=184$ as could have been expected from both the $\delta\left\langle r^{2}\right\rangle$ jump observed in this experiment and the change in ground state spin and parity just mentioned. As a conclusion, it is clear that it is for the Ir isotopes with a mass lower than (or equal to) 186 that our approach is the most legitimate. Our conclusions concerning the heavier isotopes will have to be taken keeping in mind the present caveat paragraph.

\subsection{The odd nuclei $A=183,185,187,189,191,193$}

Since we were bound to strictly axial symmetry, we had to decide whether a prolate or an oblate shape should be assumed. One way to decide was to perform an unconstrained calculation for both signs of deformation and compare the obtained quadrupole and magnetic moments to the experimental results. These quantities are indeed very sensitive to the deformation sign since the intrinsic quadrupole moment has opposite sign and the magnetic moment value is generally very sensitive to the exact nature of the single particle orbitals involved in the nuclear state. This comparison is reported as an example in Tab. 6 for ${ }^{193} \mathrm{Ir}$ using a ${ }^{192}$ Os core. The observed positive sign of the spectroscopic moment is only obtained in the prolate calculation. In addition, the only way to reproduce a magnetic moment value as small as the observed one is 
Table 6. Results of the rotor +1 qp calculations for ${ }^{193} \mathrm{Ir}$ using a ${ }^{192}$ Os core with deformation values at equilibrium for both the prolate and oblate shapes. The states are labeled as described in paragraph 4.1.

\begin{tabular}{|c|c|c|}
\hline${ }^{192}$ Os core & oblate & prolate \\
\hline total energy $(\mathrm{MeV})$ & -1515.4262 & -1516.3704 \\
\hline deformation & $\beta_{2}=-0.160$ & $\beta_{2}=+0.154$ \\
\hline states & $\frac{3}{2}^{+} \frac{3}{2}^{+}[431]$ & $\frac{3}{2} \frac{3}{2}^{+}[402]$ \\
\hline Coriolis mixing & $90 \%\left\langle\left\langle{ }^{\mathrm{HF}} \psi_{\frac{3}{2} \frac{3}{2}}\right\rangle+9.4 \%\left\langle{ }^{\mathrm{HF}} \psi_{\frac{3}{2} \frac{1}{2}}\right\rangle+\ldots\right.$ & $99.6 \%\left\langle{ }^{\mathrm{HF}} \psi_{\frac{3}{2} \frac{3}{2}}\right\rangle+0.4 \%\left\langle{ }^{\mathrm{HF}} \psi_{\frac{3}{2} \frac{1}{2}}\right\rangle$ \\
\hline $\mathrm{Q}_{s_{\text {calc }}}(\mathrm{b})$ & -0.731 & +0.866 \\
\hline $\mathrm{Q}_{s_{\text {exp }}}(\mathrm{b})$ & \multicolumn{2}{|c|}{$\begin{array}{l}+0.75(7) \text { (this work) } \\
+0.751(9)([32])\end{array}$} \\
\hline \multirow[b]{2}{*}{$\mu_{\text {calc }}\left(\mu_{N}\right)$} & $0.6 \mathrm{~g}_{s_{\text {free }}}$ & $0.6 \mathrm{~g}_{s_{\text {free }}}$ \\
\hline & +1.114 & -0.156 \\
\hline$\mu_{e x p}\left(\mu_{N}\right)$ & \multicolumn{2}{|c|}{$\begin{array}{c}+0.166(7)(\text { this work }) \\
+0.1637(6)[32]\end{array}$} \\
\hline
\end{tabular}

Table 7. Calculated and experimental values of the magnetic and spectroscopic quadrupole moments for the odd Ir isotopes. The first values are those from literature and the second ones, those obtained in this experiment. The references are the same as in tabs. 3 and $4 .{ }^{*}$ note: the unpaired particle contribution has been neglected (see text).

\begin{tabular}{|c|c|c|c|c|c|c|c|}
\hline \multirow{3}{*}{$\begin{array}{c}\text { nucleus } \\
I K^{\pi}\left[N n_{3} \Lambda\right]\end{array}$} & \multirow{3}{*}{$\begin{array}{c}\beta_{2} \\
\text { (from IS) }\end{array}$} & \multirow{3}{*}{$\begin{array}{c}\text { core } \\
\beta_{2}\end{array}$} & $\mu^{e x p}\left(\mu_{N}\right)$ & \multicolumn{2}{|c|}{$\mu^{t h}\left(\mu_{N}\right)$} & \multirow{3}{*}{$\begin{array}{l}Q_{s}^{\exp }(\mathrm{b}) \\
\text { literature } \\
\text { this work }\end{array}$} & \multirow{3}{*}{$\frac{Q_{s}^{t h}(\mathrm{~b})}{\text { rotor }+1 \mathrm{qp}^{*}}$} \\
\hline & & & literature & \multicolumn{2}{|c|}{ rotor $+1 q p$} & & \\
\hline & & & this work & $g_{s}^{\text {free }}$ & $0.6 g_{s}^{\text {free }}$ & & \\
\hline $\begin{array}{c}{ }^{193} \mathrm{Ir} \\
3 / 23 / 2^{+}[402]\end{array}$ & +0.144 & $\begin{array}{l}{ }^{192} \mathrm{Os} \\
+0.141\end{array}$ & $\begin{array}{c}+0.1636(6) \\
+0.168(8)\end{array}$ & -0.147 & +0.485 & $\begin{array}{c}+0.751(9) \\
+0.73(7)\end{array}$ & +0.788 \\
\hline 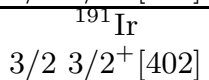 & +0.155 & $\begin{array}{l}{ }^{190} \mathrm{Os} \\
+0.155\end{array}$ & $\begin{array}{c}+0.1507(6) \\
+0.152(4)\end{array}$ & -0.154 & +0.482 & $\begin{array}{c}+0.816(9) \\
+0.82(8)\end{array}$ & +0.867 \\
\hline 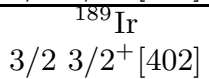 & +0.163 & 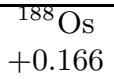 & $\begin{array}{c}0.13(4) \\
+0.147(7)\end{array}$ & -0.153 & +0.482 & $\begin{array}{c}+0.878(10) \\
+0.85(7)\end{array}$ & +0.924 \\
\hline 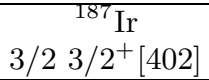 & +0.167 & $\begin{array}{r}{ }^{186} \mathrm{Os} \\
+0.173\end{array}$ & $\begin{array}{c}- \\
+0.17(1)\end{array}$ & -0.151 & +0.485 & $\begin{array}{c}+0.941(11) \\
+0.9(1)\end{array}$ & +0.960 \\
\hline 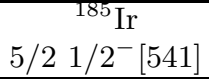 & +0.202 & $\begin{array}{r}{ }^{184} \mathrm{Os} \\
+0.206\end{array}$ & $\begin{array}{c}2.605(13) \\
+2.59(7)\end{array}$ & +1.38 & +2.06 & $\begin{array}{c}-2.06(14) \\
-1.7(6)\end{array}$ & -1.46 \\
\hline 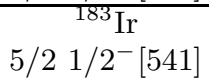 & +0.207 & $\begin{array}{l}{ }^{182} \mathrm{Os} \\
+0.207\end{array}$ & $\begin{array}{c}- \\
+2.40(8)\end{array}$ & +1.37 & +2.02 & $\begin{array}{c}- \\
-1.8(7)\end{array}$ & -1.51 \\
\hline
\end{tabular}

for a positive deformation. In that case, it appears that this value is very sensitive to the effective $g_{s}$; however the experimental value lies in between the two limits adopted for the calculation $0.6 g_{s}^{\text {free }}$ and $g_{s}^{\text {free }}$. Finally it can be noted that the binding energy of the ground state is higher in the prolate case. This comparison is qualitatively the same for $A=191,189$ and 187 using respectively the Os core with $\mathrm{A}=190,188$ and 186 . Therefore, a prolate shape was assumed in the axial calculations for all the even cores in order to describe the ground states of the odd Ir nuclei from $\mathrm{A}=183$ to 193 .

Once the sign of the deformation was determined, the calculations were done at different deformation values (constrained or equilibrium) for the even Os cores with $\mathrm{A}=182$ to 192 and the even $\mathrm{Pt}$ cores with $\mathrm{A}=184$ to 194 . As an illustration, the sp energies in the Hartree-Fock field of $182,184,186$ Os are plotted as a function of the deformation in Fig. 16. The sp orbitals located above the Fermi level will obviously be involved in the Ir low-lying level description. The relative energies of the band heads calculated using the rotor+1qp approach are represented in Fig. 17. With the Os cores, the calculations predict a $5 / 2^{+}[402]$ state as the ground state. However, the occupation probability of this state, about 0.9 , indicates that it is expected to be located at low energy in the Re nuclei but not in the Ir ones, which is actually observed. The $5 / 2^{+}$state is indeed the ground state of the odd ${ }^{179-189}$ Re nuclei. The first qp state with $v^{2} \leq 0.5$, i.e. expected at low energy in the Ir nuclei, is the $3 / 2^{+}[402]$ or $5 / 21 / 2^{-}$[541] state for the Os cores with $\mathrm{A} \geq 184$ or $\mathrm{A}=182$ and 184 respectively. This is in agreement with the experimental spin and parity values. The qp states obtained with the Pt cores are generally empty $\left(v^{2}<0.5\right)$ and consequently better suited to describe the states of the Au nuclei. Therefore, the experimental magnetic and spectroscopic quadrupole moments are compared with the values calculated using the Os qp wave functions. These different values are reported in Tab. 7 .

\subsubsection{Analysis of the magnetic moments}

For ${ }^{187,189,191,193} \mathrm{Ir}$, despite the great sensitivity of the calculated magnetic moments to the variation of $g_{s}^{\text {eff }}$ which has already been noted above, the low absolute value and the sign of the moments can be reproduced only for a $3 / 2^{+}[402]$ state. The best possibility to account for the ${ }^{183} \mathrm{Ir}$ and ${ }^{185} \mathrm{Ir}$ experimental magnetic moment values is 
neutron single-particle levels
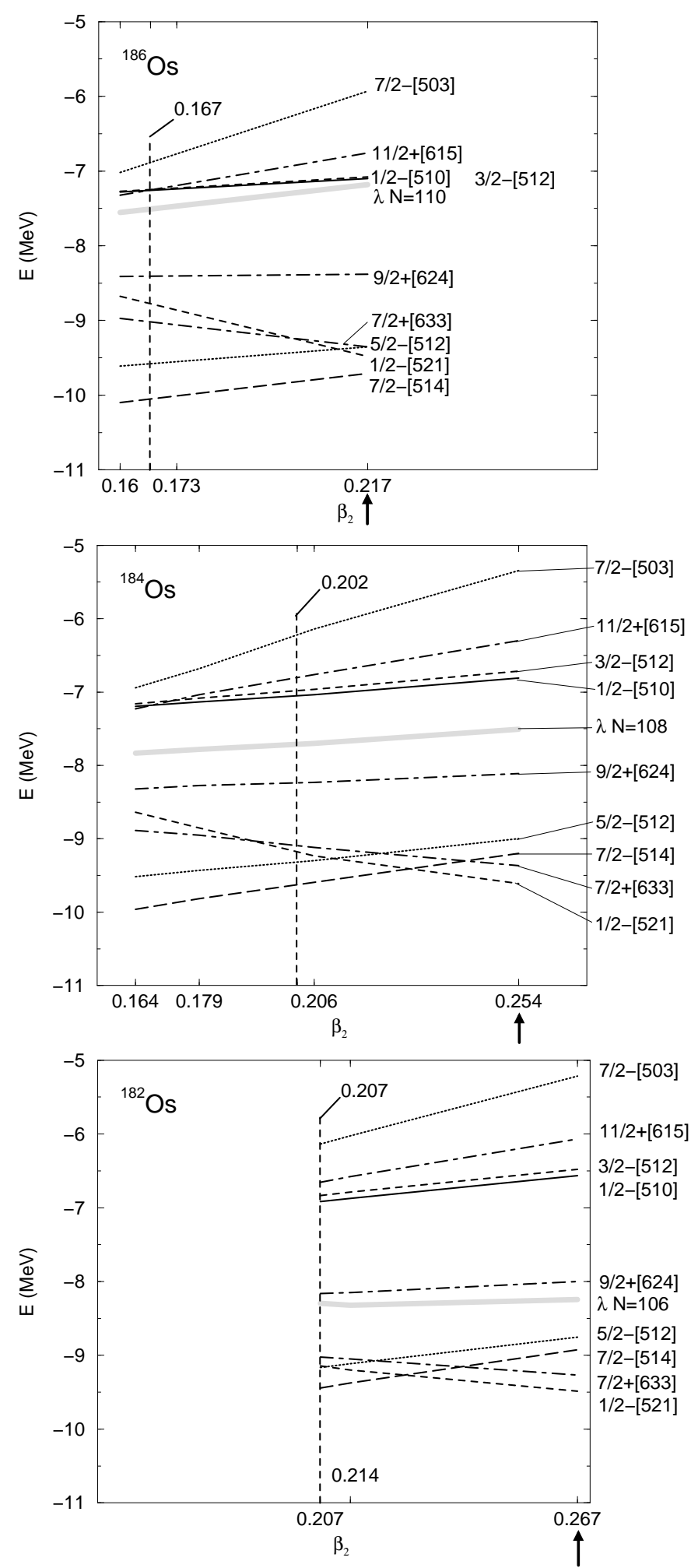

proton single-particle levels
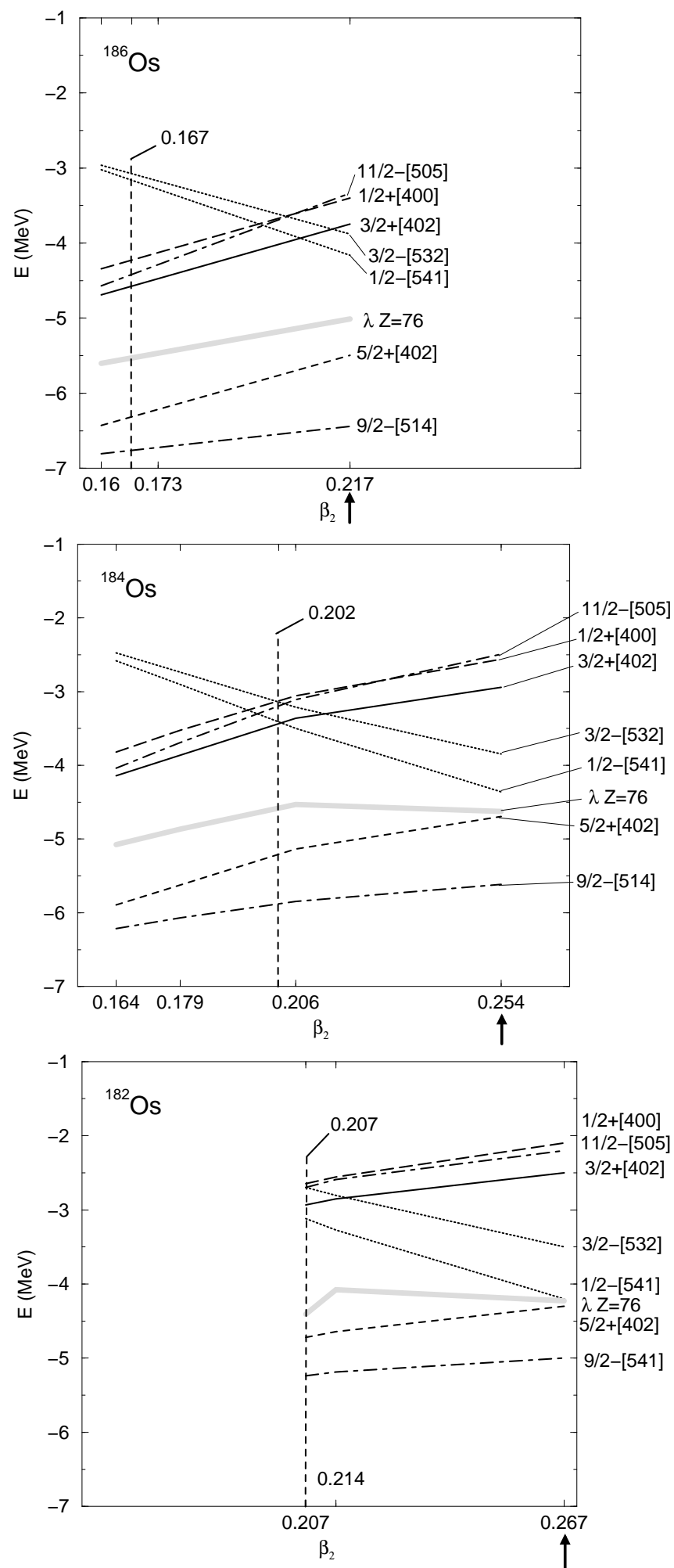

Fig. 16. Energies of the neutron and proton sp-states in the HF field of the even-even cores ${ }^{186} \mathrm{Os}(\mathrm{N}=110)$, ${ }^{184} \mathrm{Os}(\mathrm{N}=108)$ and ${ }^{182} \mathrm{Os}(\mathrm{N}=106)$ as a function of the axial deformation parameter. The Fermi levels are indicated with thick grey lines. The values of the axial deformation parameter at which the calculations were performed are indicated on the horizontal axis. The deformation values obtained for equilibrium (constraint free calculations) are indicated by arrows, those of the odd Ir nuclei deduced from the IS measurements are indicated by vertical dotted lines. 


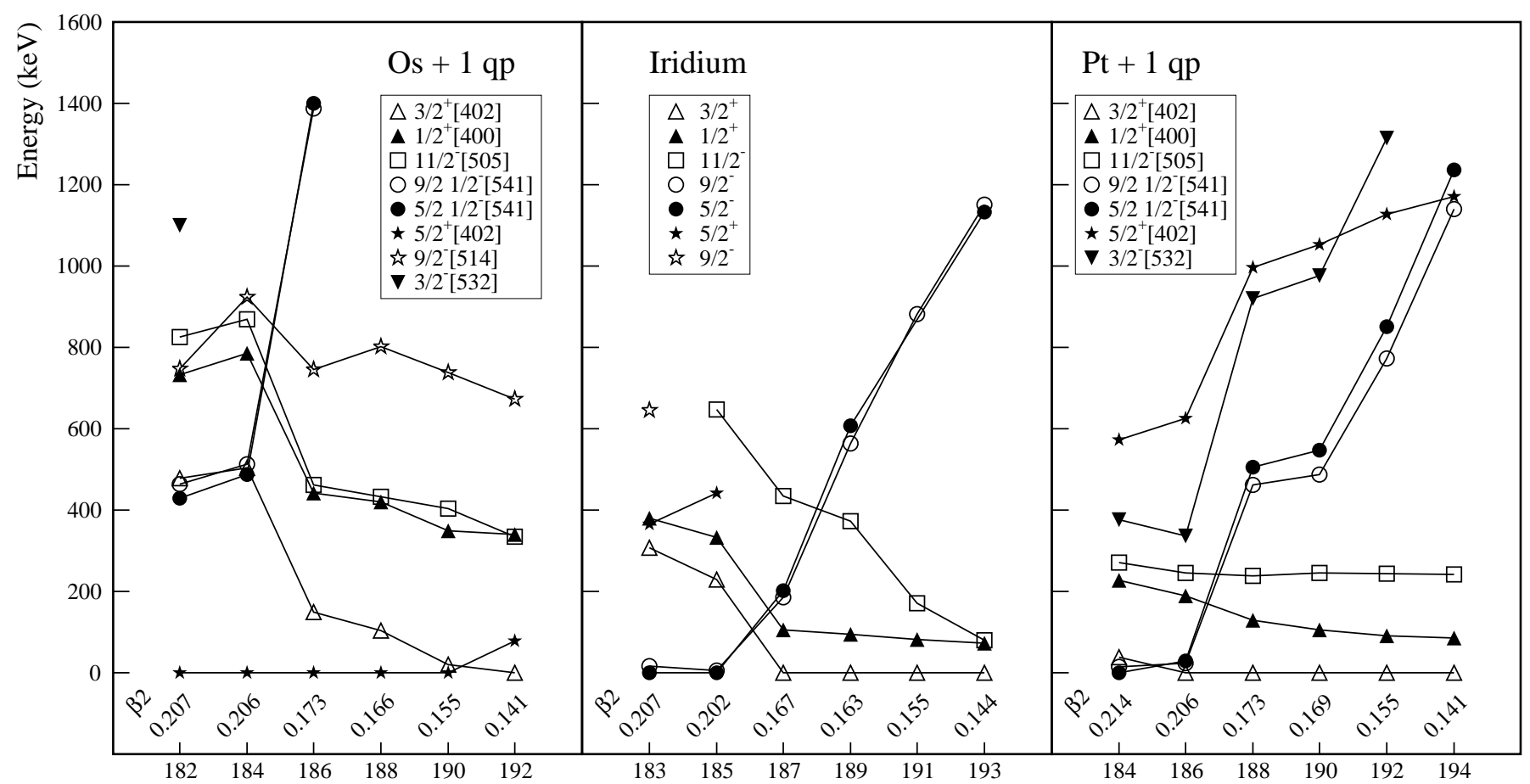

Fig. 17. First states of the rotational bands observed in the odd isotopes ${ }^{183-193}$ Ir compared to the results of the rotor $+1 \mathrm{qp}$ calculations using the corresponding ${ }^{182-192}$ Os and ${ }^{184-194} \mathrm{Pt}$ even cores. The calculations were done for deformation values close to those extracted from the IS measurement. The $\beta_{2}$ values are reported at the bottom of the graphs.

by considering a $5 / 21 / 2^{-}[541]$ state. In that case, as can be seen in Tab. 7, in order to improve the agreement with the experimental values it is necessary to use a very depressed $g_{s}^{e f f}$ factor, even lower than $0.6 g_{s}^{\text {free }}$. This would be the evidence of a very strong polarization effect. It is interesting to notice that the discrepancy between the calculated and observed $\mu$ values has previously been discussed, at least in the case of ${ }^{185} \mathrm{Ir}$ : see [35] and references therein. The polarization in particular has been pointed out recurrently either directly or not. For instance, considering a pure Nilsson 5/2 1/2-[541] orbital the calculated ${ }^{185}$ Ir magnetic moment obtained by Ekström et al. [65] is $\mu^{\text {calc }}=+1.31 \mu_{N}$ for $g_{s}=g_{s}^{\text {free }}$ and $\mu^{\text {calc }}=+1.76 \mu_{N}$ for $g_{s}=0.6 g_{s}^{f r e e}$. Van Walle et al. performed a Nilsson + pairing + Coriolis mixing calculation for $\beta_{2} \simeq 0.20-0.25$ and a low value for the effective $g_{s}: g_{s}^{e f f}=0.5-0.6 g_{s}^{\text {free }}$. In those conditions they obtained a value between $+2.2 \mu_{N}$ and $+2.5 \mu_{N}$ [76] for a $5 / 2^{-}$state having a component of $90 \%$ on $1 / 2^{-}$[541] and $10 \%$ on $3 / 2^{-}$[532]. From this result they concluded that the experimental magnetic moment could only be accounted for by considering the Coriolis mixing. Ohya et al. also made an estimate for $\mu$ taking into account the influence of the Coriolis effects by considering an effective magnetic decoupling parameter $b_{0}^{\text {eff }}$ deduced from the effective decoupling parameter $a_{e f f}$ of the experimental rotational band. The obtained value is in good agreement with the experiment if a low $g_{s}^{\text {eff }}$ is used: $\mu^{\text {calc }}=+2.5 \mu_{N}$ for $g_{s}=0.6 g_{s}^{\text {free }}$ [35]. The agreement between the experimental ${ }^{185} \mathrm{Ir}$ rotational band built on the $5 / 2^{-}$state and the result of our calculations is quite sat- isfactory as can be seen in Fig. 18. This means that the the Coriolis effects are well reproduced in our description. Our calculation using the ${ }^{184}$ Os core at $\beta_{2}=+0.206$ gives $5 / 2 " 1 / 2^{-}[541] "=85.5 \%\left\langle 1 / 2^{-}[541]\right\rangle+14.5 \%\left\langle 3 / 2^{-}[532]\right\rangle$. This is consistent with the result of van Walle et al.. We can also confirm that the increase of the Coriolis mixing could lead to an increase of the magnetic moment value: for instance in the case of $5 / 2^{-}$state obtained with a ${ }^{186} \mathrm{Pt}$ core at $\beta_{2}=+0.206$, the Coriolis mixing is higher: $5 / 2 " 1 / 2^{-}[541] "=78.4 \%\left\langle 1 / 2^{-}[541]\right\rangle+21.6 \%\left\langle 3 / 2^{-}[532]\right\rangle$ which gives for the magnetic moment: $\mu^{\text {calc }}=+1.39 \mu_{N}$ and $\mu^{\text {calc }}=+2.10 \mu_{N}$ for $g_{s}=g_{s}^{\text {free }}$ and $g_{s}=0.6 g_{s}^{\text {free }}$ respectively. The maximum value which we could obtain in our calculations was $\mu^{\text {calc }}=+2.17 \mu_{N}$ using a ${ }^{186} \mathrm{Pt}$ core at $\beta_{2}=+0.155$ with $g_{s}=0.6 g_{s}^{\text {free }}$ corresponding with a $81 \% 1 / 2^{-}[541]$ wave function.

It is therefore tempting to conclude that the origin of the increased value of the ${ }^{185} \mathrm{Ir}$ magnetic moment as compared to what could be expected from a pure Nilsson $1 / 2^{-}[541]$ orbital is twofold: $(i)$ strong Coriolis effects and (ii) a very low effective $g_{s}$ value of about $0.5-0.6 g_{s}^{\text {free }}$ which could be the indication of a strong polarization effect. We will see in par. 4.4 that we have reasons to think that those two effects are correlated. It is clear from both the values of Tab. 7 and the good agreement between theory and experiment in Fig. 18, that the same conclusions can be drawn for ${ }^{183} \mathrm{Ir}$. 

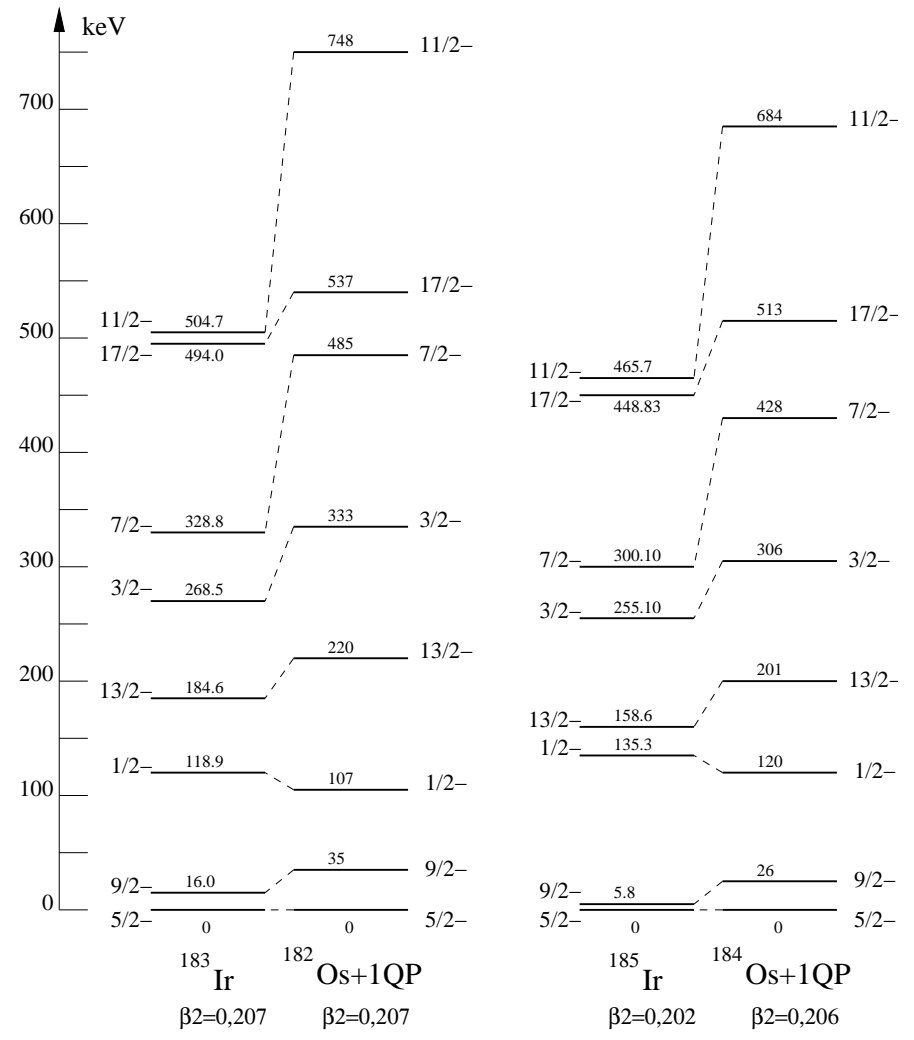

Fig. 18. Lower part of the experimental and calculated ground-state rotational bands for ${ }^{183} \mathrm{Ir}$ and ${ }^{185} \mathrm{Ir}$. The deformation parameters extracted from the IS measurement as well as the calculated Os core and constraint deformations are indicated at the bottom of the level schemes.

\subsubsection{Analysis of the spectroscopic quadrupole moments}

The analysis of the quadrupole moments should provide more detailed information on the ground state wave functions and especially on their composition on the standard basis originating from the Coriolis mixing. From the proton sp diagram in Fig. 16 we expect some mixing between the $3 / 2^{+}[402]$ and $1 / 2^{+}[400]$ orbitals for the positive parity states and the $1 / 2^{-}[541]$ and $3 / 2^{-}[532]$ for the negative parity states. In the adiabatic (or strong) coupling limit for the rotor, the spectroscopic $Q_{s}$ and intrinsic $Q_{0}$ quadrupole moments are related by the simple relation :

$$
Q_{s}=\frac{3 K^{2}-I(I+1)}{(I+1)(2 I+3)} Q_{0}
$$

Furthermore, the deformation parameter $\beta_{2}$ corresponds to the moment of order two of the intrinsic shape and is related to $Q_{0}$ by [77]:

$$
Q_{0}=\frac{3}{\sqrt{5 \pi}} Z R_{0}^{2} \beta_{2}\left(1+\frac{2}{7} \sqrt{\frac{5}{\pi}} \beta_{2}+\frac{1}{14 \pi} \beta_{2}^{2}-\ldots\right)
$$

We have restricted this development to the second order. Then it is possible to estimate the spectroscopic quadrupole moment assuming different pure $K$ states and using the
Table 8. Spectroscopic quadrupole moments for different assumed $K$ values and using $Q_{0(I S)}$ (see text). The experimental values are given again for comparison. They are taken from literature when the precision is better than ours (cf Tab. 4) that is in every case except ${ }^{183} \mathrm{Ir}$ for which no value was available before our experiment.

\begin{tabular}{|c|c|c|}
\hline nucleus & $K^{\pi}$ & $Q_{s(I S)}(\mathrm{b})$ \\
\hline${ }^{193} \mathrm{Ir}$ & $1 / 2^{+}$ & -0.849 \\
& $3 / 2^{+}$ & +0.849 \\
\hline${ }^{191} \mathrm{Ir}$ & $1 / 2^{+}$ & -0.911 \\
& $3 / 2^{+}$ & +0.911 \\
\hline${ }^{189} \mathrm{Ir}$ & $1 / 2^{+}$ & -0.954 \\
& $3 / 2^{+}$ & +0.954 \\
\hline${ }^{187} \mathrm{Ir}$ & $1 / 2^{+}$ & -0.972 \\
& $3 / 2^{+}$ & +0.972 \\
\hline${ }^{185} \mathrm{Ir}$ & $1 / 2^{-}$ & -1.69 \\
& $3 / 2^{-}$ & -0.42 \\
\hline${ }^{183} \mathrm{Ir}$ & $1 / 2^{-}$ & -1.72 \\
& $3 / 2^{-}$ & -0.43 \\
\hline
\end{tabular}$\quad$\begin{tabular}{|} 
\\
\hline
\end{tabular}

$Q_{0(I S)}$ values obtained with the $\left\langle\beta_{2}^{2}\right\rangle^{1 / 2}$ values extracted from the IS measurements. These "pure $K$ " values are reported in Tab. 8.

For the positive parity ground states of ${ }^{187-193} \operatorname{Ir}$ the two $Q_{s(I S)}^{K}$ values for $K=1 / 2$ and $K=3 / 2$ are of the same amplitude but have opposite signs. Then the actual value of the spectroscopic quadrupole moment can be accounted for by including a component of about $5 \%$ of the $1 / 2^{+}[400]$ orbital in the wave function. Since the two rotational bands built on the $3 / 2^{+}[402]$ and $1 / 2^{+}[400]$ Nilsson orbitals are well identified (see [78] and references therein), their mixing and the decoupling parameter (for $K=1 / 2$ ) can be extracted from the experimental spectra e.g. by applying the method of inversion of the eigenvalue problem [79]. This analysis leads to a $K=1 / 2$ component of about $7.5 \%$ which is in good agreement with the result deduced from the quadrupole moments. But it is in contrast with the results of the calculations which give a very pure ground state $3 / 2^{+}[402]$. This feature comes directly from the fact that the calculated energy separation between the $3 / 2^{+}[402]$ and $1 / 2^{+}[400]$ orbitals as seen in Fig. 16 is greater by nearly a factor 2 than the difference extracted from the rotational band analysis. This explains the slight difference between the theoretical and experimental values of $Q_{s}$ for ${ }^{187-193} \operatorname{Ir}$ (Tab. 7), but the agreement is sufficient to assign the Nilsson label.

For the negative parity ground states of ${ }^{183} \mathrm{Ir}$ and ${ }^{185} \mathrm{Ir}$ the comparison of Tab. 8 would give a very pure $K=1 / 2$ state. Unfortunately, for ${ }^{183} \mathrm{Ir}$ our uncertainties allow any possible $K=1 / 2$ and $K=3 / 2$ mixing and our result is the only value presently available. However, as shown in Fig. 19, many measurements have been performed on ${ }^{185} \mathrm{Ir}$. The weighted average $(-2.09 \pm 0.11)$ exceeds (in absolute value) the limit for a completely pure $K=1 / 2$ wave function, which is somewhat surprising. The $1 / 2^{-}[541]$ is an intruder state of opposite parity stemming from the $1 h_{\frac{9}{2}}$ spherical orbital above the $\mathrm{Z}=82$ gap so that no other $K=1 / 2$ negative parity orbital is available for mixing. If 


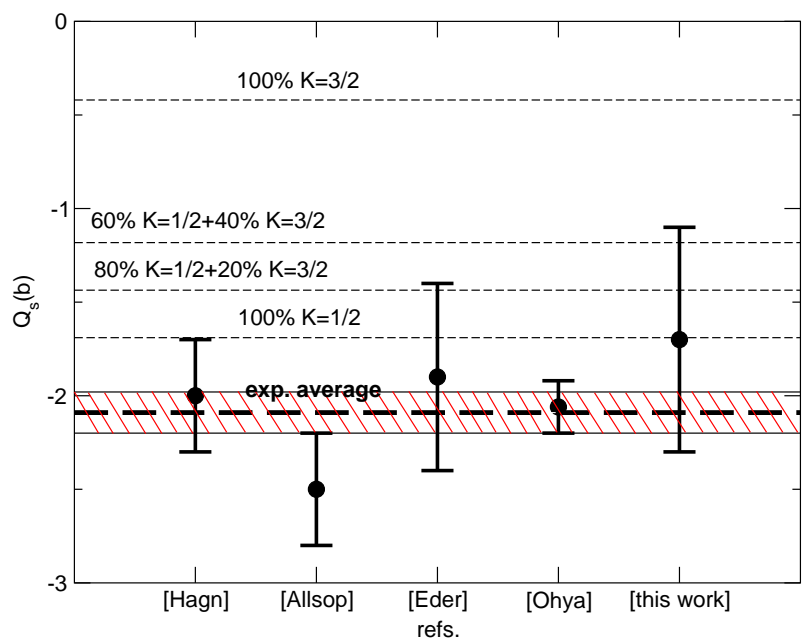

Fig. 19. Experimental $Q_{s}$ values for ${ }^{185}$ Ir available in the literature: from Static nuclear Orientation with $\gamma$ detection: Hagn et al. [43] and Allsop et al. [44] and from Nuclear Magnetic Resonance on Oriented Nuclei: Eder et al. [80] and Ohya et al. [35]. A thick dashed line indicates the weighted average of the experimental values and the hatched zone represents the probable error of the mean. The others thin dashed lines correspond to different $K$ mixing situations.

the result of this $Q_{s}$ analysis indicates a $100 \% 1 / 2^{-}[541]$ wave function for the ${ }^{185} \mathrm{Ir}$ ground state then the perturbation of the rotational band must originate exclusively from the diagonal Coriolis term proportionally to the decoupling parameter. This is in fact impossible: the maximum value for this parameter corresponding with the situation of a completely decoupled proton motion i.e. a pure $1 h_{\frac{9}{2}}$ valence proton orbital, is $a=+5$ as a result of the expression for this parameter in the spherical basis :

$$
a=\sum_{j}(-1)^{j-\frac{1}{2}}\left(j+\frac{1}{2}\right) C_{\Omega=\frac{1}{2} j}^{2}
$$

taking $C_{\Omega=\frac{1}{2} j}$ the component on the $\left|\Omega=\frac{1}{2} j\right\rangle$ spherical orbital equal to one. Actually our calculations for ${ }^{185} \mathrm{Ir}$ correspond to a well decoupled (rotation aligned) situation with $a=+4.43$ for ${ }^{183} \operatorname{Ir}\left({ }^{182} \mathrm{Os}\right.$ core and $\left.\beta_{2}=+0.207\right)$ and $a=+4.49$ for ${ }^{185} \operatorname{Ir}\left({ }^{184} \mathrm{Os}+1 \mathrm{qp}\right.$ and $\left.\beta_{2}=+0.206\right)$. Another calculation using the Nilsson model made for ${ }^{185} \mathrm{Ir}$ by André et al. [81] which fits the experimental levels gives an even higher value: $a=+4.8$. The upper limit for the decoupling parameter would lead to a $5 / 2$ band-head and a closed first excited level $\mathrm{I}=1 / 2$. This is in contrast with the experimental situation as shown in Fig. 18 where we can see a very low $\mathrm{I}=9 / 2$ first excited state. The experimental situation of a quasi-degeneracy between the $\mathrm{I}=5 / 2$ ground state and $\mathrm{I}=9 / 2$ corresponds to a much higher effective decoupling parameter $a_{e f f} \simeq+7.5$ which is the evidence of a supplementary perturbation coming from the non-diagonal term of the Coriolis interaction. The good agreement which is observed in Fig. 18 between the calculated and experimental rotational bands (especially the lower part) confirms this statement and as shown in the previous paragraph the ground state wave function contains a significant amount of the $3 / 2^{-}[532]$ orbital (e.g. $14.5 \%$ for ${ }^{185} \mathrm{Ir}$ ). However, one must not forget that the reference for the deformation parameter is $\beta_{2}=+0.155$ for ${ }^{191}$ Ir. The disconcerting result of the $Q_{s}$ analysis could simply originate from the fact that this reference value was underestimated. But if the core deformation is increased in the calculations the $3 / 2^{-}$[532] component in the wave function tends to be lower, resulting in a serious worsening of the rotational band aspect while the improvement of the calculated $Q_{s}$ value is meaningless.

The traditional analysis of $Q_{s}$ seems to be misleading for the two odd nuclei ${ }^{183} \mathrm{Ir}$ and ${ }^{185} \mathrm{Ir}$. As in the case of the strong magnetic moment suppression observed for those two nuclei, a tentative explanation for this anomalous feature will be proposed in section 4.4.

\subsection{The odd-odd nuclei $A=182,184,186,186^{m}, 188$}

The determination of the neutron-proton configuration dominating the ground and long-lived states of these oddodd nuclei has generally been subject to many discussions in both in-beam and radioactivity $\gamma$-spectroscopy works. We hope to provide here some supplementary indications on the nature of isomeric and ground states by analyzing our results on the nuclear moments. We can undertake the study of the quadrupole moments as before to determine the $K$ values and if possible put an upper limit on the Coriolis mixing. This is done in Tab. 9 in a similar way as in Tab. 8: a prolate intrinsic shape was assumed except in the case of ${ }^{188}$ Ir where there is still room for doubt. Furthermore we compare the experimental magnetic moments to the calculated values in order to discriminate the best candidates for the proton-neutron configurations. As mentioned previously the present development of the model for the odd-odd nuclei does not allow the calculation of the spectroscopic quadrupole moments for the doubly-odd nuclei. The magnetic moments were only calculated for pure $K$ proton-neutron configuration states.

${ }^{188}$ Ir The ${ }^{188} \mathrm{Ir}$ ground state was assigned a $I=1$ spin by Eder et al. [33]. Kreiner et al. have constructed a zeroorder approximation level scheme from the experimental energies of the low lying neutron state in the isotone ${ }^{187} \mathrm{Os}$ and the interpolated proton energies from the two odd neighbouring Ir nuclei ${ }^{185} \mathrm{Ir}$ and ${ }^{187} \operatorname{Ir}$ [82]. From this they suggest a configuration $\nu 1 / 2^{-}[510] \otimes \pi 3 / 2^{+}[402]$ for the ground state. This procedure implicitly assumes a prolate shape for this state and we have no further indication that would completely rule out the possibility of an oblate shape. In the hypothesis of an oblate shape one can expect from Tab. 9 a $K$-mixed $I=1$ state with $68 \%(K=0)+32 \%(K=1)$. Oblate states with $K=1 / 2$ which could lead to such a mixed $I=1$ state in the oddodd case do appear in the calculations at low energy. For instance, using a ${ }^{188} \mathrm{Pt}$ core with $\beta_{2}=-0.172$, one obtains for the neutrons a $1 / 2^{-}$band head of the perturbed $\nu 1 / 2^{-}[530]$ band at $476 \mathrm{keV}$ and for the protons a $11 / 2^{-}$ 
Table 10. Magnetic moments calculated for pure (see text) neutron-proton configurations using the single particle states in the Os even cores and assuming different $K$ values. Comparison with the experimental results from literature (refs. are in Tab. 3 ) and this work.

\begin{tabular}{|c|c|c|c|c|c|c|}
\hline \multirow[t]{2}{*}{ nucleus / core $\left(\beta_{2}\right)$} & \multirow[t]{2}{*}{ configuration } & \multirow[t]{2}{*}{$K$} & \multicolumn{2}{|c|}{$\mu\left(\mu_{N}\right)($ th. $)$} & \multicolumn{2}{|c|}{$\mu\left(\mu_{N}\right)(\exp )}$. \\
\hline & & & \multicolumn{2}{|c|}{\begin{tabular}{l|l}
$g_{s}^{\text {free }}$ & $0.6 g_{s}^{\text {free }}$
\end{tabular}} & \multirow{2}{*}{$\begin{array}{c}\text { literature } \\
0.302\end{array}$} & \multirow{2}{*}{$\frac{\text { this work }}{+0.31(1)}$} \\
\hline${ }^{188} \operatorname{Ir} /{ }^{186}$ Os $(0.173)$ & $\begin{array}{l}\nu 1 / 2^{-}[510] \otimes \pi 3 / 2^{+}[402] \\
\nu 3 / 2^{-}[512] \otimes \pi 1 / 2^{+}[400]\end{array}$ & $\begin{array}{l}K=1 \\
K=1\end{array}$ & $\begin{array}{l}+0.49 \\
-0.51\end{array}$ & $\begin{array}{l}+0.77 \\
-0.23\end{array}$ & & \\
\hline \multirow[t]{2}{*}{${ }^{186} \mathrm{Ir}^{g} / /{ }^{184} \mathrm{Os}(0.206)$} & $\nu 1 / 2^{-}[510] \otimes \pi 1 / 2^{-}[541]$ & $\begin{aligned} & K=0 \\
& K=1 \\
& 50 \%[K=0]+50 \%[K=1]\end{aligned}$ & $\begin{array}{l}+2.09 \\
+1.88 \\
+3.93\end{array}$ & $\begin{array}{l}+2.09 \\
+1.98 \\
+3.87\end{array}$ & \multirow[t]{2}{*}{$3.88(5)$} & \multirow[t]{2}{*}{$+3.7(2)$} \\
\hline & $\nu 1 / 2^{-}[521] \otimes \pi 1 / 2^{-}[541]$ & $\begin{aligned} & K=0 \\
& K=1 \\
& 50 \%[K=0]+50 \%[K=1]\end{aligned}$ & $\begin{array}{l}+2.09 \\
+2.11 \\
+2.79\end{array}$ & $\begin{array}{l}+2.09 \\
+2.11 \\
+3.00\end{array}$ & & \\
\hline \multirow[t]{5}{*}{${ }^{186} \mathrm{Ir}^{m} /{ }^{184}$ Os $\left.(0.164)\right)$} & $\nu 1 / 2^{-}[510] \otimes \pi 3 / 2^{+}[402]$ & $\begin{array}{l}K=1 \\
K=2\end{array}$ & $\begin{array}{l}+0.87 \\
-0.98\end{array}$ & $\begin{array}{l}+1.1 \\
+0.05\end{array}$ & \multirow[t]{5}{*}{$0.638(8)$} & \multirow[t]{5}{*}{$-0.63(3)$} \\
\hline & $\nu 1 / 2^{-}[521] \otimes \pi 3 / 2^{+}[402]$ & $\begin{array}{l}K=1 \\
K=2\end{array}$ & $\begin{array}{l}+0.51 \\
-0.28\end{array}$ & $\begin{array}{l}+0.85 \\
+0.47\end{array}$ & & \\
\hline & $\nu 7 / 2^{-}[503] \otimes \pi 3 / 2^{+}[402]$ & $K=2$ & -0.50 & -0.72 & & \\
\hline & $\nu 3 / 2^{-}[512] \otimes \pi 3 / 2^{+}[402]$ & $K=0$ & \multicolumn{2}{|c|}{$\frac{1}{+0.84}$} & & \\
\hline & $\nu 3 / 2^{-}[512] \otimes \pi 1 / 2^{+}[400]$ & $\begin{array}{l}K=1 \\
K=2\end{array}$ & $\begin{array}{l}+0.21 \\
+2.9\end{array}$ & $\begin{array}{c}+0.40 \\
+1.9\end{array}$ & & \\
\hline${ }^{184} \operatorname{Ir} /{ }^{182}$ Os $(0.214)$ & $\nu 9 / 2^{+}[624] \otimes \pi 1 / 2^{-}[541]$ & $\begin{array}{l}K=4 \\
K=5\end{array}$ & $\begin{array}{l}-0.23 \\
-0.81\end{array}$ & $\begin{array}{l}+0.10 \\
-0.16\end{array}$ & $0.696(5)$ & $+0.69(3)$ \\
\hline${ }^{182} \mathrm{Ir} /{ }^{180} \mathrm{Os}(0.202)$ & $\nu 1 / 2^{-}[521] \otimes \pi 1 / 2^{-}[541]$ & $\begin{array}{c}K=0 \\
K=1 \\
50 \%[K=0]+50 \%[K=1]\end{array}$ & $\begin{array}{r}+ \\
+1.34 \\
+1.98\end{array}$ & $\begin{array}{r}.27 \\
+1.32 \\
+2.72\end{array}$ & $2.28(8)$ & $+2.64(19)$ \\
\hline
\end{tabular}

band head of the mixed $\pi\left\{1 / 2^{-}[530], 1 / 2^{-}[521]\right\}$ band at $342 \mathrm{keV}$. On the other hand, as was shown in Ref. [83] there is no firm experimental evidence of oblate states in the odd nuclei of this region. Therefore we will restrict ourselves to the prolate hypothesis, which indicates a very pure $K=1$ wave function. From the previous analysis on the odd isotopes we can expect a $\pi 3 / 2^{+}[402]$ proton state with a significant contribution of the $\pi 1 / 2^{+}[400]$ orbital. Besides, the two rotational bands built on the neutron sp states $\nu 1 / 2^{-}[510]$ and $\nu 3 / 2^{-}$[512] are known to be intimately mixed in the neighbouring odd-neutron nuclei. This is especially true in the isotone ${ }^{187}$ Os [84] where the analysis of the level scheme leads to a situation where these two single particle states are quasidegenerate. Thus five components are expected to be involved to describe the $\mathrm{I}=1^{188} \mathrm{Ir}$ ground state: the favored $\nu 1 / 2^{-}[510] \otimes \pi 3 / 2^{+}[402]$ configuration with $\mathrm{K}=1$, the favored and unfavored $\nu 1 / 2^{-}[510] \otimes \pi 1 / 2^{+}[400]$ configuration with $\mathrm{K}=1$ and $\mathrm{K}=0$ respectively, the unfavored $\nu 3 / 2^{-}[512] \otimes \pi 3 / 2^{+}[402]$ configuration with $\mathrm{K}=0$ and the favored $\nu 3 / 2^{-}[512] \otimes \pi 1 / 2^{+}[400]$ with $\mathrm{K}=1$. The three favored components which can contribute have $\mathrm{K}=1$, which could explain the pure $\mathrm{K}=1$ state indicated by the measured $Q_{s}$ value. The rotor+2qp hamiltonian has been diagonalized in the space constituted by the proton and
Table 9. Same as Tab. 8 for the odd-odd isotopes.

\begin{tabular}{|c|c|c|c|c|}
\hline nucleus & $Q_{0(I S)}(\mathrm{b})$ & $\bar{K}$ & $Q_{s(I S)}(\mathrm{b})$ & $Q_{s(\exp )}(\mathrm{b})$ \\
\hline \multirow{4}{*}{$\begin{array}{l}{ }^{188} \mathrm{Ir} \\
(\mathrm{I}=1)\end{array}$} & \multirow[t]{2}{*}{+4.66} & 0 & -0.932 & \multirow[t]{4}{*}{$+0.484(6)$} \\
\hline & & 1 & +0.466 & \\
\hline & \multirow[t]{2}{*}{-4.66} & 0 & +0.932 & \\
\hline & & 1 & -0.466 & \\
\hline \multirow{6}{*}{$\begin{array}{l}{ }^{186} \mathrm{Ir}^{g} \\
(\mathrm{I}=5)\end{array}$} & \multirow{6}{*}{+5.93} & 0 & -2281 & \multirow{6}{*}{$-2.548(31)$} \\
\hline & & 1 & 2053 & \\
\hline & & $\begin{array}{l}1 \\
2\end{array}$ & $\begin{array}{l}-2.000 \\
-1368\end{array}$ & \\
\hline & & 3 & -0.228 & \\
\hline & & 4 & +1.368 & \\
\hline & & 5 & +3.421 & \\
\hline \multirow{3}{*}{$\begin{array}{c}{ }^{186} \mathrm{Ir}^{m} \\
(\mathrm{I}=2)\end{array}$} & \multirow{3}{*}{+4.87} & 8 & & \multirow{3}{*}{$+1.456(17)$} \\
\hline & & 0 & -1.391 & \\
\hline & & $\begin{array}{l}1 \\
2\end{array}$ & $\begin{array}{r}-0.090 \\
+1.391\end{array}$ & \\
\hline & & & & \\
\hline \multirow{6}{*}{$\begin{array}{l}{ }^{184} \mathrm{Ir} \\
(\mathrm{I}=5)\end{array}$} & \multirow[t]{6}{*}{+5.88} & 0 & -2.262 & \multirow[t]{6}{*}{$+2.1(3)$} \\
\hline & & 1 & -2.035 & \\
\hline & & 2 & -1.357 & \\
\hline & & 3 & -0.226 & \\
\hline & & 4 & +1.357 & \\
\hline & & 5 & +3.392 & \\
\hline \multirow{4}{*}{$\begin{array}{l}{ }^{182} \mathrm{Ir} \\
(\mathrm{I}=3)\end{array}$} & \multirow{4}{*}{+5.84} & $\overline{0}$ & -1947 & \multirow{4}{*}{$-1.7(6)$} \\
\hline & & 1 & -1.460 & \\
\hline & & 2 & 0 & \\
\hline & & 3 & +2.433 & \\
\hline
\end{tabular}


neutron qp states which are expected at low energy

$\begin{aligned} \text { neutron qp states: } & \text { proton qp states: } \\ \nu 9 / 2^{+}[624] & \pi 1 / 2^{+}[400] \\ \nu 11 / 2^{+}[615] & \pi 3 / 2^{+}[402] \\ \nu 1 / 2^{-}[521] & \pi 1 / 2^{-}[541] \\ \nu 1 / 2^{-}[510] & \pi 3 / 2^{-}[532] \\ \nu 3 / 2^{-}[512] & \\ \nu 7 / 2^{-}[503] & \end{aligned}$

When the residual proton-neutron interaction is not included, the ground state is predicted to be a $1^{-}$state of main component on $\nu 1 / 2^{-}[510] \otimes \pi 3 / 2^{+}[402]$ :

$$
\begin{aligned}
\left\langle 1^{-}\right\rangle & =66.2 \%\left\langle\nu 1 / 2^{-}[510] \otimes \pi 3 / 2^{+}[402]\right\rangle & K=1 \\
& +33.7 \%\left\langle\nu 3 / 2^{-}[512] \otimes \pi 3 / 2^{+}[402]\right\rangle & K=0
\end{aligned}
$$

When the residual proton-neutron interaction is included, the $\nu 7 / 2^{-}[503] \otimes \pi 3 / 2^{+}[402]$ configuration becomes the ground state with a band head $\mathrm{I}=2$ while the $1^{-}\left[\nu 1 / 2^{-}[510]\right.$ $\left.\otimes \pi 3 / 2^{+}[402]\right]$ level is pushed $145 \mathrm{keV}$ above. In some calculations using other cores we have also obtained such a phenomenon where a configuration involving the $\nu 7 / 2^{-}[503]$ orbital is favored by the residual proton-neutron interaction. This phenomenon is not well understood. The main component of the calculated $I=1$ band head located at the lowest energy in the calculation including the residual proton-neutron interaction is $\nu 1 / 2^{-}[510] \otimes \pi 3 / 2^{+}[402]$ :

$$
\begin{aligned}
\left\langle 1^{-}\right\rangle & =82.6 \%\left\langle\nu 1 / 2^{-}[510] \otimes \pi 3 / 2^{+}[402]\right\rangle & K=1 \\
& +14.8 \%\left\langle\nu 3 / 2^{-}[512] \otimes \pi 1 / 2^{+}[400]\right\rangle & K=1
\end{aligned}
$$

The magnetic moment calculated for those two $K=1$ states are given in Tab. 10. The agreement is consistent with a $\nu 1 / 2^{-}[510] \otimes \pi 3 / 2^{+}[402]$ major component and $g_{s} \simeq g_{s}^{\text {free }}$.

In conclusion, for ${ }^{188} \mathrm{Ir}$ a good agreement between all the experimental results and the calculated ones is obtained for a prolate shape and a $\nu 1 / 2^{-}[510] \otimes \pi 3 / 2^{+}[402]$ configuration as proposed by Kreiner et al. [82].

${ }^{186} \mathbf{I r}^{g}$ The mass 186 is the exact point where the sudden increase of the deformation occurs. A high spin value of $I=5$ has first been determined for the ground state of ${ }^{186}$ Ir from the ABMR method [85]. Later on, the ground state was mainly associated with $I^{\pi}=5^{+}$in most of the literature (see e.g. [86]). The assignment of $K$ has been more debatable [87], but it was concluded that the ${ }^{186} \mathrm{Ir}$ ground state should correspond to a strong mixing of $K=0$ and $K=1$ [87]. Kreiner et al. have then clearly identified a $\Delta I=2$ rotational band above the ground state [88] characteristic of a strong double-decoupling situation which cannot appear but for a prolate shape. The neutron-proton configuration of the ground state has been widely discussed in the past in order to explain this double-decoupling feature. A strong doubledecoupling situation mainly originates from the non-diagonal part of the Coriolis interaction which connects the two $K=0$ and $K=1$ states of the same proton-neutron configuration involving two $\Omega=\frac{1}{2}$ intrinsic states:

$$
\begin{aligned}
\left\langle\frac{1}{2} \frac{1}{2} K=0\right| \mathcal{H}_{\text {cor }} & \left|\frac{1}{2} \frac{1}{2} K=1\right\rangle= \\
& -\frac{\hbar^{2}}{2 \Im_{0}} \sqrt{I(I+1)}\left[a_{n}+(-1)^{(I+1)} a_{p}\right]
\end{aligned}
$$

The nature of the proton state was understood to be the $\pi 1 / 2^{-}$[541], orbital which we confirm since the measured deformation parameter $\beta_{2}=(+) 0.202$ corresponds to a situation where this orbital is brought down to the ground state (see figs. 16 and 17). The nature of the neutron state was more questionable. In order to explain the doublydecoupled character of a rotational band both the proton and the neutron must be decoupled, that is $\left|a_{p}\right| \geq 1$ and $\left|a_{n}\right| \geq 1$, as was shown by Emery et al. [89]. Therefore $\Omega=\frac{1}{2}$ neutron states situated in the vicinity of the Fermi level are suggested. As can be seen in Fig. 16 the only $\Omega=\frac{1}{2}$ neutron orbitals available close to the Fermi level are $1 / 2^{-}[510]$ and $1 / 2^{-}[521]$. At first sight the $1 / 2^{-}[510]$ is not a good candidate because the decoupling parameter of the $1 / 2^{-}[510]$ band in the isotone ${ }^{185} \mathrm{Os}$ is almost zero [84] and this very small value is confirmed in our calculation using a ${ }^{184}$ Os core with $\beta_{2}=+0.206$ which gives $a=+0.05$. A better candidate could then be the $1 / 2^{-}[521]$ neutron state because the $1 / 2^{-}[521]$ bands which are identified in some neighboring nuclei are known to be well decoupled, our calculated decoupling parameter of this band is indeed $a=+0.92$. But a further analysis shows that there are many arguments against this candidate. First the experimental evidence of such a state in ${ }^{185} \mathrm{Os}$ is reported only once at about $400 \mathrm{keV}$ [90] and was never confirmed subsequently. The first state of the $1 / 2^{-}[521]$ band is predicted at $733 \mathrm{keV}$ in our calculation using the ${ }^{184}$ Os core at $\beta_{2}=+0.206$. It would then be necessary to imagine an effect able to bring this orbital down in energy when it is coupled to the proton state in the deformed ${ }^{186} \mathrm{Ir}$ ground state. As will be seen later there is no evidence of such a strong specific neutron-proton interaction effect leading to this lowering in the calculations. The deformation is not a favouring parameter either. It appears in Fig. 16 that the energy of this hole qp state is strongly unfavored by the deformation, unlike the $1 / 2^{-}[541]$ proton qp which has the opposite particle nature. This does not favor a coupling between this two states. Finally, it has to be mentioned that, even at low deformation, due to its hole nature, $1 / 2^{-}[521]$ barely mixes with $1 / 2^{-}[510]$ through the closest $K=3 / 2$ states which are and because they are particle states. This means that no supplementary perturbation in the $1 / 2^{-}[510]$ band can be expected from a mixing with this higher lying $1 / 2^{-}[521]$ states. To better understand the situation we performed a rotor $+2 q p$ calculation including $V_{p n}$ in the following proton 
and neutron qp space

$\begin{aligned} \text { neutron qp states: } & \text { and proton qp states: } \\ \nu 9 / 2^{+}[624] & \pi 1 / 2^{+}[400] \\ \nu 11 / 2^{+}[615] & \pi 3 / 2^{+}[402] \\ \nu 1 / 2^{-}[521] & \pi 1 / 2^{-}[541] \\ \nu 1 / 2^{-}[510] & \pi 3 / 2^{-}[532] \\ \nu 3 / 2^{-}[512] & \\ \nu 7 / 2^{-}[503] & \end{aligned}$

The lowest $5^{+}$state calculated with an ${ }^{184}$ Os core at deformation $\beta_{2}=+0.206$ has the following configuration structure:

$$
\begin{array}{rlr}
\left\langle 5^{+}\right\rangle= & 24.4 \%\left\langle\nu 1 / 2^{-}[510] \otimes \pi 1 / 2^{-}[541]\right\rangle & K=0 \\
+ & 13.1 \%\left\langle\nu 1 / 2^{-}[510] \otimes \pi 1 / 2^{-}[541]\right\rangle & K=1 \\
& +9.1 \%\left\langle\nu 1 / 2^{-}[510] \otimes \pi 3 / 2^{-}[532]\right\rangle & K=1 \\
+ & 19.4 \%\left\langle\nu 3 / 2^{-}[512] \otimes \pi 1 / 2^{-}[541]\right\rangle & K=1 \\
+ & 18.9 \%\left\langle\nu 3 / 2^{-}[512] \otimes \pi 1 / 2^{-}[541]\right\rangle & K=2 \\
& +7.0 \%\left\langle\nu 3 / 2^{-}[512] \otimes \pi 3 / 2^{-}[532]\right\rangle & K=0
\end{array}
$$

that is:

$$
\begin{array}{ll}
K=0 & 31.4 \% \\
K=1 & 41.6 \% \\
K=2 & 18.9 \%
\end{array}
$$

It is interesting to notice that there is no $\nu 1 / 2^{-}[521]$ component in this $5^{+}$state. The main proton contributions are:

$$
\begin{array}{ll}
\pi 1 / 2^{-}[541] & 75.8 \% \\
\pi 3 / 2^{-}[532] & 16.1 \%
\end{array}
$$

and for the neutrons:

$$
\begin{array}{ll}
\pi 1 / 2^{-}[510] & 46.6 \% \\
\pi 3 / 2^{-}[512] & 45.3 \%
\end{array}
$$

Therefore the neutron state is a completely mixed $\mathrm{K}=1 / 2$ $\mathrm{K}=3 / 2$ state. The calculated rotational bands built on the two configurations $\nu 1 / 2^{-}[521] \otimes \pi 1 / 2^{-}[541]$ and $\nu 1 / 2^{-}[510]$ $\otimes \pi 1 / 2^{-}[541]$ are compared to the experimental levels in Fig. 20.The quality of the agreement between experimental and theoretical results is similar for the two calculated configurations: the $3^{+}, 2^{+}, 5^{+}$and $4^{+}$are almost degenerate, the band head is $3^{+}$instead of $5^{+}$and the band perturbations are similar. However the $\nu 1 / 2^{-}[521]$ $\otimes \pi 1 / 2^{-}[541]$ band head is calculated at $1292 \mathrm{keV}$ above the $\nu 1 / 2^{-}[510] \otimes \pi 1 / 2^{-}[541]$. The calculations strongly point towards a $\nu 1 / 2^{-}[510] \otimes \pi 1 / 2^{-}[541]$ configuration for the ${ }^{186} \mathrm{Ir} 5^{+}$ground state as the band head of a doublydecoupled band. The fact that the $\nu 1 / 2^{-}[510]$ could lead to the desired supplementary perturbation necessary to create this double-decoupling feature could be considered as unexpected but this was already suggested by Kreiner

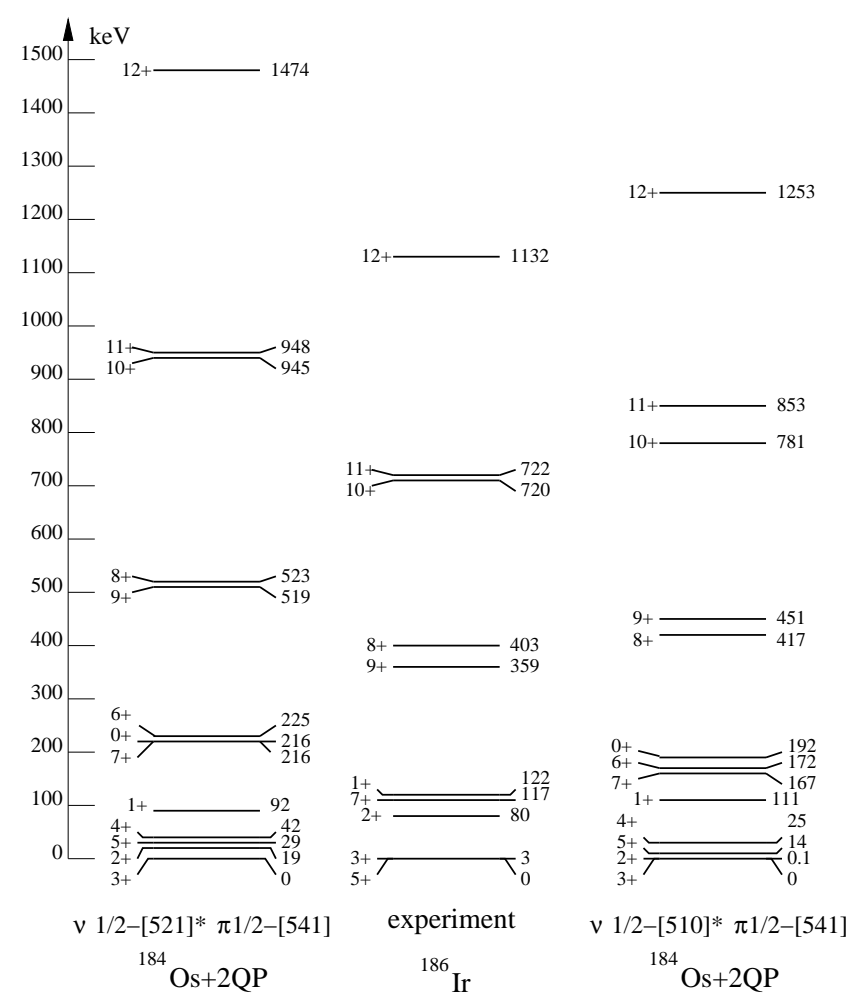

Fig. 20. The two calculated band built on the $\nu 1 / 2^{-}[521] \otimes$ $\pi 1 / 2^{-}[541]$ and the $\nu 1 / 2^{-}[510] \otimes \pi 1 / 2^{-}[541]$ main configurations compared with the experimental ${ }^{186}$ Ir ground state band. A ${ }^{184}$ Os core at $\beta_{2}=+0.206$ was used.

[91]. He showed that due to their strong mixing the two neutron orbitals $1 / 2^{-}[510]$ and $3 / 2^{-}$[512] fulfill the conditions of a $\left[\widetilde{411}, 1 \pm \frac{1}{2}\right]$ pseudo-spin pair [91]. The favored member of the doublet acts as a $j=\frac{1}{2}$ particle which aligns on the rotation giving rise to the double-decoupling phenomenon. More recently the second member of the doublet has been shown experimentally, confirming the correctness of this description [93]. This complete mixing between this two neutron orbitals emerges quite naturally from our calculations which then tend to confirm a $\nu\left\{1 / 2^{-}[510], 3 / 2^{-}[512]\right\} \otimes \pi 1 / 2^{-}[541]$ for the ground state in good agreement with the previous works on this nucleus.

A complete neutron state mixing is only reproduced when the proton-neutron interaction $V_{p n}$ is included in the calculations. To illustrate this, the energy levels of the states with their main components on the $\nu 1 / 2^{-}[510] \otimes$ $\pi 1 / 2^{-}[541]$ and $\nu 3 / 2^{-}[512] \otimes \pi 1 / 2^{-}[541]$ configurations are represented in Fig. 21 for the calculations including $V_{p n}$ or excluding it. They are compared with the experimental levels from Ref. [60]. The calculation with simple geometrical coupling gives a $3^{+}$ground state with an $I=4$ state situated just a few $\mathrm{keV}$ above. The contributions to the neutron state come from the $\nu 1 / 2^{-}[510]$ orbital at $78.1 \%$ and from the $\nu 3 / 2^{-}[512]$ orbital at $17.4 \%$ only 

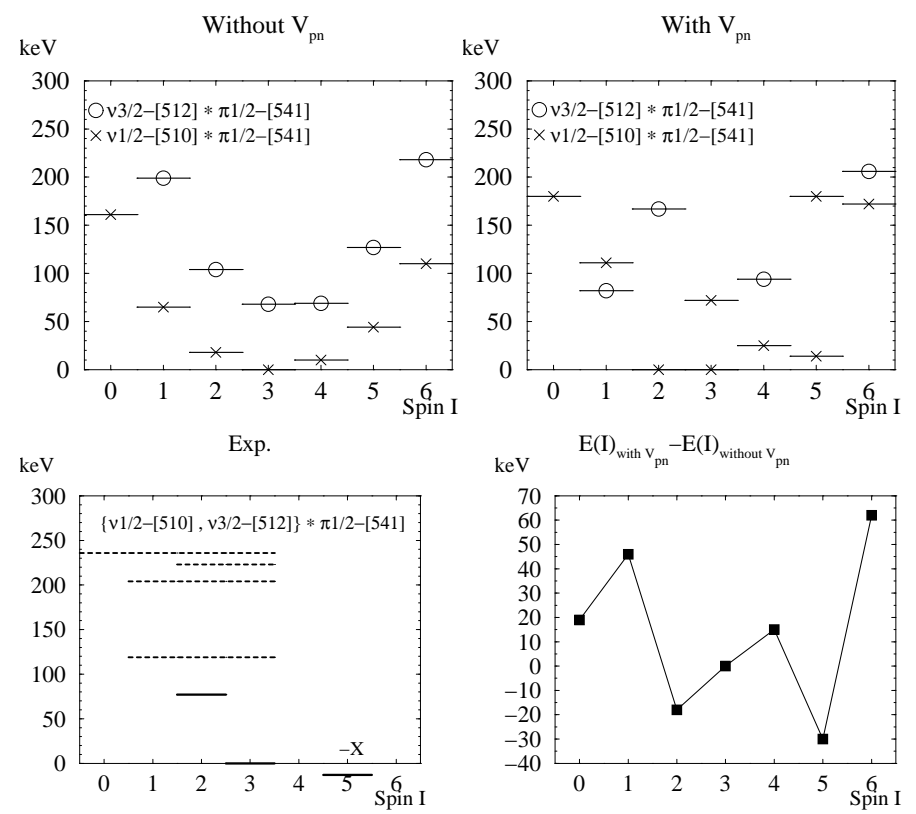

Fig. 21. Upper part: first levels with their main component on $\nu 1 / 2^{-}[510] \otimes \pi 1 / 2^{-}[541]$ and on $\nu 3 / 2^{-}[512] \otimes \pi 1 / 2^{-}[541]$ calculated with a ${ }^{184}$ Os core with a constraint of $\beta_{2}=0.206$ with and without including the residual proton-neutron interaction. Lower part: experimental levels from [92]. When the spin assignment is uncertain, the levels are represented with dashed lines covering the range of the proposed values. On lower right are represented the difference in energy between the first level of the $\nu 1 / 2^{-}[510] \otimes \pi 1 / 2^{-}[541]$ band calculated with and without the neutron-proton interaction.

(to be compared with the $46.6 \%$ and $45.3 \%$ ratios given above). Interestingly, when the residual proton-neutron is included, this $I=4$ level is pushed upwards while the $I=5$ level is brought to less than $10 \mathrm{keV}$ above the $I=3$ state which remains the ground state. As can be seen on the lower right picture, the $I=5$ state is particularly favored by the residual proton-neutron interaction with respect to the levels with different spin values. This point is very important since such a direct effect on the lowering of the $I=5$ state of this doubly-decoupled band has never been indicated in any previous calculations although it was felt to be necessary $[89,88]$. The use of other cores and other deformations to create various mixings confirms that the higher spin levels are generally favored in energy for a maximum decoupling of the $1 / 2^{-}[541]$ proton plus a neutron in a strongly mixed $\left\{1 / 2^{-}[510], 3 / 2^{-}[512]\right\}$ state.

It is tempting to claim that the neutron state mixing which is the base of the double-decoupling situation originates from proton-neutron residual interaction. It is now interesting to see how the measured values of the magnetic and spectroscopic moments take place in the complex picture we have just depicted in detail. We have calculated the magnetic moment values for both pure configurations $\nu 1 / 2^{-}[510] \otimes \pi 1 / 2^{-}[541]$ and $\nu 1 / 2^{-}[521] \otimes \pi 1 / 2^{-}[541]$. They are reported in Tab. 10 for the three situations $100 \%$ $K=0,100 \% K=1$ and $50 \%(K=0)+50 \%(K=1)$, the latter giving the highest $\mu$ value. It is clear, as al- ready suggested in Ref. [87], that the only way to reach the experimental value is to assume a maximum mixing. This is a clear indication that the Coriolis effect is very important. On the other hand, one sees on Tab. 9 that the comparison between the measured spectroscopic moment and the spectroscopic moments obtained from the intrinsic prolate deformation indicates a very pure $K=0$ state. As we shall see later in section 4.4 this apparent disagreement probably originates from the same mechanism as in the odd ${ }^{185} \mathrm{Ir}$ case.

${ }^{186}{ } \mathbf{r r}^{m}$ From the $\beta$-NMR method a spin $I=2$ was assigned to the isomeric state of ${ }^{186} \operatorname{Ir}$ [94]. Ben Braham et al. [92] have performed an analysis of the level scheme of ${ }^{186}$ Ir using a zero-order scheme constructed from the experimental energies of the low lying neutron states in the isotone ${ }^{185} \mathrm{Os}$ and the interpolated proton energies from the two odd neighboring Ir nuclei ${ }^{185} \mathrm{Ir}$ and ${ }^{187} \mathrm{Ir}$. From these considerations, they propose two prolate configurations for the isomeric state: $\nu 1 / 2^{-}[510] \otimes \pi 3 / 2^{+}[402]$ or $\nu 7 / 2^{-}[503] \otimes \pi 3 / 2^{+}[402]$, the second being the most probable. That the $3 / 2^{+}[402]$ intrinsic proton state should be involved in this isomeric state is consistent with our determination of the deformation parameter $\beta_{2}=(+) 0.167$ which corresponds to a situation where it is lower in energy than $1 / 2^{-}[541]$ as we saw before. However, there may be other candidates for the neutron state: using a ${ }^{184}$ Os core constrained to a deformation $\beta_{2}=+0.164$, one sees that the $1 / 2^{-}[521]$ and $3 / 2^{-}[521]$ neutron states also come very close to the Fermi level. From the comparison between the experimental quadrupole moment and the value obtained using the intrinsic deformation and different values for $K$ (Tab. 9) one sees that the wave function should be of a very pure $K=2$ nature. To discriminate between these different possible neutron states, we have calculated the magnetic moment for the possible proton-neutron configurations for different pure $K$ assumptions. These values are reported in Tab. 10. The sign of the magnetic moment which is determined here for the first time points towards the $\nu 7 / 2^{-}[503] \otimes \pi 3 / 2^{+}[402]$ configuration. The $\left[\nu 1 / 2^{-}[510] \otimes \pi 3 / 2^{+}[402]\right]_{K=2}$ could still constitute a reasonable candidate. In the rotor $+2 q p$ calculations the diagonalisation was done in the same qp space as for ${ }^{186} \mathrm{Ir}^{g}$. The lowest lying $2^{-}$states obtained in that space are situated at $89 \mathrm{keV}$ :

$$
\begin{aligned}
\left\langle 2^{-}\right\rangle_{1} & =55.4 \%\left\langle\nu 3 / 2^{-}[512] \otimes \pi 3 / 2^{+}[402]\right\rangle & & K=0 \\
& +31.0 \%\left\langle\nu 1 / 2^{-}[510] \otimes \pi 1 / 2^{+}[400]\right\rangle & & K=0 \\
& +11.7 \%\left\langle\nu 1 / 2^{-}[510] \otimes \pi 3 / 2^{+}[402]\right\rangle & & K=1
\end{aligned}
$$

at $154 \mathrm{keV}$ :

$$
\begin{aligned}
\left\langle 2^{-}\right\rangle_{2}= & 60.7 \%\left\langle\nu 1 / 2^{-}[510] \otimes \pi 3 / 2^{+}[402]\right\rangle & K=1 \\
+ & 24.8 \%\left\langle\nu 3 / 2^{-}[512] \otimes \pi 1 / 2^{+}[400]\right\rangle & K=1 \\
& +6.6 \%\left\langle\nu 1 / 2^{-}[510] \otimes \pi 1 / 2^{+}[400]\right\rangle & K=0 \\
& +5.8 \%\left\langle\nu 3 / 2^{-}[512] \otimes \pi 3 / 2^{+}[402]\right\rangle & K=0
\end{aligned}
$$


and at $169 \mathrm{keV}$ :

$$
\left\langle 2^{-}\right\rangle_{3}=99.6 \%\left\langle\nu 7 / 2^{-}[503] \otimes \pi 3 / 2^{+}[402]\right\rangle \quad K=2
$$

The $\left\langle 2^{-}\right\rangle_{3}$ state is the only one that corresponds to a pure $K=2$ state. Furthermore it is the band head of the $\nu 7 / 2^{-}[503] \otimes \pi 3 / 2^{+}[402]$ configuration whereas the bandhead of the two other configurations are a $0^{-}$state at 0 $\mathrm{keV}$ for the first and a $1^{-}$state at $76 \mathrm{keV}$ for the second. Therefore, the sign of the magnetic moment, the analysis of the quadrupole moment and the result of the calculation definitely indicate a $\nu 7 / 2^{-}[503] \otimes \pi 3 / 2^{+}[402]$ configuration for the $I=2$ isomeric state of ${ }^{186} \mathrm{Ir}$.

${ }^{184}$ Ir The exact value of the ground state spin of ${ }^{184}$ Ir has been discussed, both $I=4$ and $I=5$ being proposed [43, $95,97,96]$. In our case of an atomic transition between the $J=9 / 2$ and $J=11 / 2$ hyperfine multiplets, the number of hyperfine transitions is 24 if $I=4$ while it is 29 if $I=5$. So far, we have identified 25 peaks, but it is sometimes difficult to conclude firmly on the weakest. Then our experimental results strongly suggest a value of $I=5$.

In an in-beam experiment Kreiner et al. have identified a perturbed rotational structure built on the ground state characterized by a succession of $\Delta I=1$ transitions [98]. Such a Coriolis perturbed band is characteristic of a semi - decoupled situation [99] where the motion of one of the unpaired particle aligns to the rotation of the core while the other stays well coupled. This situation can arise if the $1 / 2^{-}[541]$ proton state intervenes in the ground state. This is compatible with the deformation value extracted from the IS which is $\beta_{2}=(+) 0.202$ and then would be of positive sign. The case of an oblate ground state is indeed highly improbable since it would imply a sudden and violent shape transition with respect to the two prolate neighboring odd isotopes ${ }^{183} \mathrm{Ir}$ and ${ }^{185} \mathrm{Ir}$ and there is no indication of such a situation in the $\gamma$-spectroscopy studies. The comparison of the experimental quadrupole moment and the different values obtained from the intrinsic prolate shape as shown in Tab. 9 indicates a mixed $K=4$ and $K=5$ wave function with a possible component on $K=3$. The only available neutron orbital susceptible to couple to the $K=1 / 2$ proton state to give such $K$ values is $9 / 2^{+}[624]\left(1 i_{\frac{13}{2}}\right.$ parentage) which is identified in the two neighbouring odd-neutron isotones ${ }^{183} \mathrm{Os}$ and ${ }^{185} \mathrm{Pt}$. A $\nu 9 / 2^{+}[624] \otimes \pi 1 / 2^{-}[541]$ configuration was proposed in order to give an interpretation of the low lying levels of ${ }^{184} \mathrm{Ir}$ fed by the radioactive decay of ${ }^{184} \mathrm{Pt}$ in Ref. [96] and confirmed from the study of the decay of ${ }^{184} \mathrm{Ir}$ to ${ }^{184} \mathrm{Os}$ in Ref. [97]. In the case of the doubly odd nuclei, when one of the unpaired particles has $K=1 / 2$, the associated decoupling parameter enters the non-diagonal Coriolis term and we can expect a large mixing of the two components $K=4$ and $K=5$ of the $\nu 9 / 2^{+}[624] \otimes \pi 1 / 2^{-}[541]$ configuration. The result of the rotor $+2 \mathrm{qp}$ calculation for the $5^{-}$ground state gives the following wave function:

$$
\begin{aligned}
\left\langle 5^{-}\right\rangle= & 50.3 \%\left\langle\nu 9 / 2^{+}[624] \otimes \pi 1 / 2^{-}[541]\right\rangle & K=5 \\
+ & 44.4 \%\left\langle\nu 9 / 2^{+}[624] \otimes \pi 1 / 2^{-}[541]\right\rangle & K=4 \\
& +5.1 \%\left\langle\nu 9 / 2^{+}[624] \otimes \pi 3 / 2^{-}[532]\right\rangle & K=3
\end{aligned}
$$

Using these percentages and the intrinsic quadrupole moment from the IS one gets $Q_{s}=+2.3 \mathrm{~b}$ which is in very good agreement with the experimental value. The calculated values of the magnetic moment for the pure $K=4$ and $K=5$ states for the $\nu 9 / 2^{+}[624] \otimes \pi 1 / 2^{-}[541]$ configuration are in disagreement with the experimental values as can be seen in Tab. 10. An improvement of the calculations would then consist in taking into account explicitly the influence of the Coriolis interaction on the magnetic moment which has to appear through the magnetic decoupling parameter $b_{0}$ associated with the $1 / 2^{-}[541]$ proton state.

${ }^{182}$ Ir Sauvage et al. have determined a spin and parity $I^{\pi}=3^{+}$for the ground state [62]. A doubly-decoupled rotational band characterized by $\Delta I=2$ transitions has also been observed. It is based on the ground state [98] which has been suggested in both references to correspond to a $\nu 1 / 2^{-}[521] \otimes \pi 1 / 2^{-}[541]$ configuration. As mentioned previously such a situation presupposes a prolate shape. In the case of ${ }^{186} \mathrm{Ir}$ with two particles coupled to a ${ }^{184} \mathrm{Os}$ core the $1 / 2^{-}[521]$ neutron state could barely play a role due to its distance from the Fermi level which increases rapidly with the deformation. For the ${ }^{182}$ Os core however, since the Fermi level has become lower, the $1 / 2^{-}[521]$ single neutron orbital is situated rather close to it for a moderate deformation - typically $\beta_{2} \simeq+0.2$, while the $1 / 2^{-}[541]$ single proton state is already at the ground state. This situation is in very nice agreement with absolute value of the deformation parameter extracted from the IS $\beta_{2}=0.202$. The comparison between the experimental quadrupole moment and the different values obtained from the intrinsic positive deformation using different values of $K$ indicates two equal contributions of $K=0$ and $K=1$ which suggest a wave function composed essentially of the $\nu 1 / 2^{-}[521] \otimes \pi 1 / 2^{-}[541]$ configuration. As in the case of ${ }^{186} \mathrm{Ir}^{g}$, we calculated the magnetic moment only for a pure configuration here $\nu 1 / 2^{-}[521] \otimes \pi 1 / 2^{-}[541]$ but it was still possible to test a different mixing of the two $K$ components of that configuration: $100 \% K=0$, $100 \% K=1$ and $50 \%(K=0)+50 \%(K=1)$ (see Tab. 10$)$. It emerges also for ${ }^{182}$ Ir that the value of the experimental moment can only be accounted for by assuming a complete mixing which underlines once more the importance of the Coriolis effect on the magnetic moment. If the Coriolis effect is important then the configuration cannot be pure because the non-diagonal Coriolis term is proportional to one of the two decoupling parameters. We know that the decoupling parameter for the $1 / 2^{-}[541]$ proton state is very large and some components in the proton state coming from other orbitals like $3 / 2^{-}[532]$ are expected to play a role. To check this point, we performed a rotor $+2 \mathrm{qp}$ calculation using a ${ }^{182}$ Os core with $\beta_{2}=+0.214$ close to 
the experimental deformation parameter. The BCS occupation factors obtained using this core are $v_{[521]}^{2}=0.93$ and $v_{[541]}^{2}=0.04$; then the $1 / 2^{-}[521]$ neutron qp is a hole state and the $1 / 2^{-}[541]$ proton qp a particle state and the coupled $\nu 1 / 2^{-}[521] \otimes \pi 1 / 2^{-}[541]$ states are believed to represent the ${ }^{182} \mathrm{Ir}$ states. The diagonalisation of the rotor+2qp hamiltonian was done in the following qp space

$$
\begin{array}{cl}
\text { for neutrons: } & \text { and for protons: } \\
\nu 9 / 2^{+}[624] & \pi 3 / 2^{+}[402] \\
\nu 1 / 2^{-}[521] & \pi 1 / 2^{-}[541] \\
\nu 1 / 2^{-}[510] & \pi 3 / 2^{-}[532] \\
\nu 3 / 2^{-}[512] & \\
\nu 7 / 2^{-}[503] & \\
\nu 7 / 2^{-}[514] &
\end{array}
$$

The resulting $3^{+}$state with main component on $\nu 1 / 2^{-}[521] \otimes_{\mathrm{S}}$ $\pi 1 / 2^{-}[541]$ is then:

$$
\begin{array}{rr}
\left\langle 3^{+}\right\rangle=40.5 \%\left\langle\nu 1 / 2^{-}[521] \otimes \pi 1 / 2^{-}[541]\right\rangle & K=0 \\
+48.6 \%\left\langle\nu 1 / 2^{-}[521] \otimes \pi 1 / 2^{-}[541]\right\rangle & K=1 \\
+5.2 \%\left\langle\nu 1 / 2^{-}[521] \otimes \pi 3 / 2^{-}[532]\right\rangle & K=2 \\
+\ldots &
\end{array}
$$

The calculation favors the component on $K=1$ and introduces a component on $K=2$. It originates as expected from the mixing between the two proton states $1 / 2^{-}[541]$ and $3 / 2^{-}[532]$. Using these calculated percentages and the $Q_{s(I S)}$ values one gets $Q_{s}=-1.5 b$ which is in agreement with the measured value. It is interesting to notice that any component coming from any other configuration than $\nu 1 / 2^{-}[521] \otimes \pi 1 / 2^{-}[541]$ leads to a lowering of the absolute value of $Q_{s}$. However, so far, a very good agreement is obtained between the calculated and the experimental results for a prolate shape and the $\nu 1 / 2^{-}[521] \otimes \pi 1 / 2^{-}[541]$ configuration.

\subsection{An analysis of the anomalous moments}

A few difficulties were emphasized in the two previous subsections concerning the analysis of the nuclear moments when the $1 / 2^{-}[541]$ proton orbital is involved in the nuclear states.

First, in the odd cases:

(i) Though the Coriolis mixing is explicitly taken into account in the calculations of the magnetic moments, the experimental values for ${ }^{183} \mathrm{Ir}$ and ${ }^{185} \mathrm{Ir}$ could not be reproduced unless a strongly depressed $g_{s}$ value of the order of 0.5-0.6 $g_{s}^{\text {free }}$ is used. This is not the case for the other odd Ir nuclei we have studied. Both the ${ }^{183} \mathrm{Ir}$ and ${ }^{185} \mathrm{Ir}$ ground states correspond to the $\mathrm{I}=5 / 2$ band head of the strongly Coriolis perturbed $1 / 2^{-}$[541] band, with, in particular, a quasi-maximum value for the decoupling parameter. This would indicate that the observed values of the magnetic moments originate from both a strong Coriolis effect and a strong suppression of the magnetic moment associated with the spin of the unpaired particle.

(ii) The spectroscopic moment $Q_{s}$ is not compatible with the absolute value of the deformation parameter extracted from the isotope shift. For ${ }^{185} \mathrm{Ir}$, the weighted average value $Q_{s}=-2.09 \pm 0.11 b$ including all the available experimental data exceeds in absolute value the expected value for a pure $K=1 / 2$ state (cf Fig. 19). For ${ }^{183} \mathrm{Ir}$, our experimental value is the only one presently available; any $K=1 / 2 K=3 / 2$ mixing is certainly allowed within the error bars but the mean value of $-1.8 b$ corresponds with a pure $K=1 / 2$ situation. This pure $K=1 / 2$ value is completely excluded for both ${ }^{185} \mathrm{Ir}$ and ${ }^{183} \mathrm{Ir}$ from the rotational band level pattern and from the calculations. Besides, the calculated $Q_{s}$ values are significantly lower in absolute value than the experimental ones.

${ }_{\text {Second, in the odd-odd cases: }}$

(iii) The Coriolis mixing is not taken into account in the magnetic moment calculation except for the $K=0$ $K=1$ mixing of the two components of the same configuration $\Omega_{p}=\frac{1}{2} \otimes \Omega_{n}=\frac{1}{2}$. For ${ }^{184}$ Ir the experimental value is not reproduced by the calculations and one may evoke the omission of the components coming from other proton-neutron configurations to explain it. For ${ }^{182} \mathrm{Ir}$ and ${ }^{186} \mathrm{Ir}^{g}$ if a pure $\Omega_{p}=\frac{1}{2} \otimes \Omega_{n}=\frac{1}{2}$ is assumed, a complete mixing $K=0 K=1$ seems to be necessary, which would indicate a maximal Coriolis effect. One cannot neglect the possibility that other configurations with $K>1$ enter the wave function in those last two cases. Besides, in ${ }^{184}$ Ir the agreement is improved when $g_{s}$ is strongly depressed. The parallelism with the point $(i)$ is striking. The ${ }^{182} \mathrm{Ir}$ and ${ }^{186} \mathrm{Ir}^{g}$ ground states are the band head of a doublydecoupled band and the ${ }^{184} \mathrm{Ir}$ ground state is the band head of a semi-decoupled band, every of those states involving the $1 / 2^{-}$[541] proton orbital.

(iv) For ${ }^{186} \mathrm{Ir}^{g}$, as in case (ii), the spectroscopic moment $Q_{s}$ is incompatible with the absolute value of the deformation parameter extracted from the isotope shift. The analysis of $Q_{s}$ leads indeed to a pure $K=0$ configuration which is not compatible with both the magnetic moment analysis and the results of the calculations. For ${ }^{182} \mathrm{Ir}$ and ${ }^{184} \mathrm{Ir}$ there is no such obvious discrepancy.

Actually, in points (ii) and (iv) everything happens as if there were some supplementary negative contribution in the observed spectroscopic quadrupole moment. For the odd nuclei, such a supplementary contribution could be understood, though in a very pictorial way, as follows. In the model of the coupling of a single particle to an axially deformed nuclear surface, the total nuclear quadrupole moment reads actually (see e.g. [100]):

$$
Q=Q_{s}+Q_{p}
$$

$Q_{s}$ is due to the motion of the nuclear surface characterized by the intrinsic deformation $Q_{0}$, the two being related 


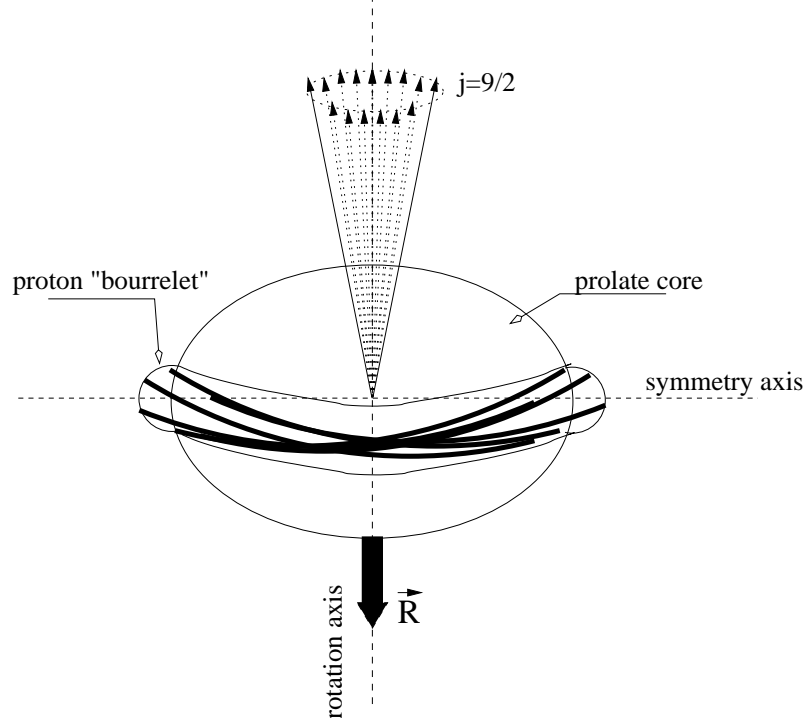

Fig. 22. Pictorial view of the "bourrelet" effect.

by Eq. 10 and $Q_{p}$ is due to the single particle motion. This second term drops in most cases where the strong coupling is a good approximation because the motion of the particle is tightly bound to the nuclear surface and does not lead to an important contribution to the total observed quadrupole moment $Q$. As we saw, there are strong indications that the proton motion is well aligned to the rotation axis i.e. decoupled from the surface motion due to the diagonal $K=1 / 2$ Coriolis interaction term. The single proton would then be essentially described by a $1 h_{\frac{9}{2}}$ wave function, its angular momentum $j=9 / 2$ being antialigned with the core rotational momentum $R=2$ to provide the total $I=5 / 2$ ground state angular momentum as shown in Fig. 22. If one considers also the fact that the radial part of a $1 h_{\frac{9}{2}}$ wave function is peaked near the nuclear surface then we arrive at a picture where the mean trajectory of the proton would describe a kind of charge bulge ("bourrelet") in the equatorial plane as shown in Fig. 22, which would naturally lead to a charge extension along the intrinsic deformation axis. This in turn would give rise to the extra contribution in the observed spectroscopic quadrupole moment we are looking for. It is very important to understand that it would be a very specific effect making the nucleus look as if it were more deformed from the laboratory frame but which would not imply a supplementary deformation of the whole core in itself. This supplementary contribution could be approximated by the single particle value of the quadrupole moment, given by (see e.g. [101]):

$$
Q_{j}=-e_{p}^{e f f} \frac{2 j-1}{2 j+2}\left\langle r^{2}\right\rangle_{N l}
$$

$e_{p}^{e f f}$ is the effective proton charge including the polarization effect:

$$
e_{p}^{e f f}=e_{p}+\alpha_{p} \frac{Z}{A} e_{p}
$$

where $e_{p}$ is the free proton charge $e_{p}=+1$ and $\alpha_{p}$ the polarization factor for proton, simply defined as $e^{e f f}=$ $Q^{\exp } /\left\langle Q^{\text {one proton }}\right\rangle$. For an orbital situated near the nuclear surface, $\left\langle r^{2}\right\rangle_{N l}$ can be estimated as

$$
\left\langle r^{2}\right\rangle \simeq\left(1.2 A^{1 / 3}\right)^{2} \cdot 10^{-2}[\mathrm{~b}]
$$

A value $\alpha_{p}=1.3$ is taken from the data on ${ }^{209} \mathrm{Bi}$. This leads for $\mathrm{A}=185$ to $Q_{p}=-0.52 \mathrm{~b}$ which has to be removed from the measured quadrupole moment in order to get the core contribution, then: $Q_{s}$ core $=-1.57(11) \mathrm{b}$ (using the weighted average values). This is the value to be used in Tab. 8 instead of $Q_{s(\text { exp })}$ for the comparison with $Q_{s(I S)}$. It allows at last, a reasonable contribution of the $3 / 2^{-}[532]$ in the ground state of the order of $10 \%( \pm 9 \%)$ in good agreement with the result of the ${ }^{184} \mathrm{Os}+1 \mathrm{qp}\left(\beta_{2}=\right.$ $+0.206)$ calculation $5 / 2 " 1 / 2^{-}[541] "=85.5 \%\left\langle 1 / 2^{-}[541]\right\rangle+$ $14.5 \%\left\langle 3 / 2^{-}[532]\right\rangle$ which in turn is consistent with the rotational band structure as we saw. The $Q_{s}$ core so obtained is also in good agreement with the result of the rotor+1qp calculation shown in Tab. 7 , consistent with the fact that the model takes into account only the collective contribution in the derivation of $Q_{s}$. Finally, a strong suppression of the magnetic moment associated with the intrinsic spin of the particle is known as negative polarization. It comes usually from a part of the residual nucleon-nucleon interaction which tends to anti-align the magnetic moment of the core and the one associated with the intrinsic spin of the unpaired nucleon. The $1 h_{\frac{9}{2}}$ is a $l-\frac{1}{2}$ orbital, if we consider the magnetic moment of the core +1 particle as (see e.g. [100]):

$$
\boldsymbol{\mu}=g_{R} \mathbf{R}+g_{l} \mathbf{l}+g_{s} \mathbf{s}
$$

then it is clear from the Fig. 22 that the magnetic moment associated with the core and the moment associated with the spin are essentially aligned. Then no special polarization effect should be expected. Let us consider the total magnetic moment of the nucleus as the result of the magnetism associated with the core motion:

$$
\mu_{R}=g_{R} R \mu_{N} \simeq \frac{Z}{A} R \mu_{N}
$$

and of the magnetism associated with a pure $1 h_{\frac{9}{2}}$ single particle orbital:

$$
\mu_{l-1 / 2}=j\left(g_{l}-\left(g_{s}-g_{l}\right) \frac{1}{2 l+1}\right) \mu_{N}
$$

The numerical values e.g. for ${ }^{185} \mathrm{Ir}$ are $\mu_{R} \simeq 0.83 \mu_{N}$ taking the approximation $g_{R}=Z / A$ and $\mu_{h_{9 / 2}}=2.62 \mu_{N}$ for $g_{s}=g_{s}^{\text {free }}$ and $\mu_{h_{9 / 2}}=3.54 \mu_{N}$ for $g_{s}=0.6 g_{s}^{\text {free }}$ taking the standard value $g_{p}^{\text {free }}=5.59$ for the free proton g-factor. Then if the two magnetic moments are considered as strictly anti-aligned, one has $\mu=1.79 \mu_{N}$ for $g_{s}=g_{s}^{\text {free }}$ and $\mu=2.71 \mu_{N}$ for $g_{s}=0.6 g_{s}^{\text {free }}$. Though it is then necessary to have a low $g_{s}^{\text {eff }}$ of about $0.65 g_{s}^{\text {free }}$ this values lies between the usual limits of $0.6-1 g_{s}^{\text {free }}$ and no specially anomalous polarization effect has to be 
invoked. Besides, this last result for the magnetic moment is far better than any of the previous calculations, which tends to confirm the correctness of the image represented in Fig. 22.

We can see now the impact of this analysis on the doubly odd nuclei. The ${ }^{186}$ Ir ground state corresponds to a situation where both the neutron and the proton motions are decoupled from the core rotation. As in the odd case, the only way to give a correct interpretation which correlates the experimental data on $Q_{s}$ and $\left|\beta_{2}\right|$ and giving a reasonable $K$ composition is to subtract from the measured $Q_{s}$ the contribution due to the uncoupled particle motions. The total nuclear quadrupole moment in the case of a single proton and a single neutron coupled to a rotor is : $Q=Q_{s}+Q_{p}+Q_{n}$. Taking for the effective neutron charge:

$$
e_{n}^{e f f}=e_{n}+\alpha_{n} \frac{Z}{A} e_{p}
$$

and assuming that the polarization effect on the core due the neutron motion is the same as that due to the proton (this is an approximation, it is probably lower, but the error is not so important since the single neutron quadrupole moment is much lower than the proton one) that is $\alpha=1.3$ as previously, then one gets $Q_{n} \simeq-0.1 b$ and $Q_{p} \simeq-0.5 b$ which give $Q_{s \text { core }} \simeq-1.95 \mathrm{~b}$. This allows indeed a strong mixing between the $K=0$ and $K=1$ components with probably a non-negligible contribution from $K=2$. When taking the proportions of the calculated $5^{+}$state which are given in the previous subsection, one obtains $Q_{s}$ core $=-1.83 b$ which is in far better agreement with the $Q_{s}$ core obtained suppressing the unpaired particle contributions than with the untreated experimental value and confirms the consistency of our analysis. For the other doubly-decoupled case ${ }^{182}$ Ir there seems to be no need to invoke such a "bourrelet" effect. Besides, the uncertainty in the value of the experimental quadrupole moment unfortunately limits a detailed analysis. However as in the previous cases we may try to give an estimation of the contribution of the two unpaired particle motions to the observed quadrupole moment. Using the same assumptions as for ${ }^{186} \mathrm{Ir}^{g}$ one gets $Q_{p} \simeq-0.5 b$ and $Q_{n} \simeq-0.1 b$, the neutron value being again probably overestimated because its radial wave function is much more spread inside the nuclear volume than the proton one due to the low- $l$ parentage of $1 / 2^{-}$[521]. This leads to an estimated $Q_{s \text { core }} \simeq-1.1(6) b$ which allows many different type of $K$ mixings up to $K=3$, but the important point is that the mean value is beyond the $K=1$ limit showing that $K \geq 2$ contribution is perhaps underestimated in our previous analysis.

At last, in the case of the semi-decoupled situation of ${ }^{184}$ Ir it is not clear whether a "bourrelet" effect should be taken into account in the analysis of the quadrupole moment. The existence of a well coupled neutron state like $9 / 2^{+}[624]$ which has, due to its $1 i_{\frac{13}{2}}$ parentage, the same property as the proton to be situated on the average, very near the nuclear surface may indeed wash out this effect due to the rotation of the core to which it is bound.
It is possible that a theoretical support for this explanation could lie for instance in an improvement of the calculations of $Q_{s}$ when the blocking effect is included and the unpaired particle contribution added. But as a first step, and especially in the case of ${ }^{184} \mathrm{Ir}$ a complete calculation of the doubly-odd nucleus magnetic moments including the neutron-proton configuration mixing due to the Coriolis effects is required. At last, one may wonder why this "bourrelet" effect was not pointed out earlier in nuclei exhibiting strongly perturbed rotational bands. From our analysis there are at least three conditions for such an effect to appear:

(i) the Coriolis effects associated with the diagonal terms have to be extremely strong and must prevail on the non diagonal terms to lead to a well rotation-aligned particle motion. This implies naturally that the unpaired particle state has a high $j$ unique parity orbital parentage (more specifically, a high $l$-parentage with its radial part strongly peaked close to the surface would lead to the strongest effect);

(ii) the decoupled unpaired particle must be a proton since the contribution from the neutron, as we saw, is much lower;

(iii) the core deformation must be small enough for the unpaired particle contribution to be felt as a supplementary charge extension in the axis plane (what we defined as the "bourrelet").

Then for instance two odd-proton nuclei in the Ir region exhibit strongly perturbed rotational bands similar to those observed in ${ }^{183,185} \mathrm{Ir}$ namely ${ }^{185} \mathrm{Au}$ and ${ }^{173} \mathrm{Ta}$. But in the former case, as the proton Fermi level is higher than for Ir, the $K=1 / 2$ and $K=3 / 2$ orbitals are equally close to it and the non-diagonal Coriolis terms are much more important i.e. condition $(i)$ is not fulfilled. In the latter case the core deformation is quite high and condition (iii) is not fulfilled.

In conclusion, other examples of the "bourrelet" effect should be looked for in prolate nuclei of transitional regions (low deformation) with their unpaired proton state characterized by a very pure $K=1 / 2$ Nilsson orbital nature.

\subsection{Summary}

The main feature of the Ir isotopic series is the sudden increase of the deformation when going away from the stability with a heavy group $187 \leq \mathrm{A} \leq 193$ characterized by deformation values in the range of $+0.15 \leq \beta_{2} \leq+0.17$ and a light group $\mathrm{A} \leq 185$ with $\beta_{2} \simeq 0.2$. The $\mathrm{A}=186$ isotope occupies an interesting intermediate position with its ground state having the deformation of the light group and its isomeric state the deformation of the heavy group. As in the gold chain there is only one jump in the evolution of the mean square charge radius but not at the same neutron number : it occurs between $\mathrm{N}=108$ and $\mathrm{N}=107$ for gold and between $\mathrm{N}=110$ and $\mathrm{N}=109$ for iridium. This jump is most probably associated with a transition from a $\gamma$-soft regime (where most probably shape-mixing dominates) to 
a sudden rigidification of the structure towards an axially symmetric prolate shape. Though the general tendency towards axial deformation when the neutron number decreases is expected from mean-field calculations (see Fig. $15)$ it appears to be enhanced by the presence of the oddproton. For $\mathrm{A} \leq 186$ the proton orbital dominating the ground state is systematically the $1 / 2^{-}[541]$ of the $1 h_{\frac{9}{2}}$ parentage which indeed enters the shell with deformation giving rise to decoupling phenomena even in the presence of an unpaired neutron and completely independently from the nature of this neutron state. Once the $\beta_{2} \simeq+0.2$ deformation is reached, the evolution of the mean square charge radius follows exactly the one given by the droplet model (see Fig. 14). This may be understood as a signature of shape stabilization due to the unpaired proton. If this is correct, it should come out naturally from more sophisticated calculations taking into account explicitly the odd number of particles. Incidentally, the motion of the unpaired proton of strong $1 h_{\frac{9}{2}}$ nature, aligned on the motion of the core gives rise to an interesting phenomenon where a large part of the observed spectroscopic quadrupole moment is due to the unpaired particle (while it was usually considered as essentially due to the core rotation). This mechanism of spectroscopic quadrupole moment enhancement - we baptized "bourrelet" effect — should be found in other mass regions provided the three conditions of Par. 4.4 happen to be fulfilled.

One of the authors (D.V.) would like to express his gratitude to Dr M.-G. Porquet for stimulating discussions and numerous helpful suggestions in particular those concerning the "bourrelet" effect and to Dr J. Libert for detailed assistance in understanding and applying the model. This work was partially supported by the Training and Mobility Program for Research and Technology of the European Community.

\section{References}

1. J. Bonn et al., Phys. Lett. 38B, (1972) 308.

2. G. Ulm et al., Z. Phys. A 325, (1986) 247.

3. K. Wallmeroth et al., Phys. Rev. Lett. 58, (1987) 1516 ; K. Wallmeroth et al., Nucl. Phys. A493, (1989) 224.

4. G. Seewald et al., Phys. Rev. Lett. 77, (1996) 5016.

5. C.R. Hammond, Handbook of Chemistry and Physics (CRC

Press, Boca Raton, Florida 1984-1985) B5

6. U. Krönert et al., NIM A 300, (1991) 522.

7. G. Savard et al., Nucl. Phys. A512, (1990) 241.

8. H.T. Duong et al., Phys. Lett. B 217, (1989) 401.

9. Th. Hilberath et al., Z. Phys. A 342, (1992) 1.

10. J.K.P. Lee et al., NIM B 34, (1988) 252.

11. F. Le Blanc et al., Phys. Rev. Lett. 79, (1997) 2213.

12. F. Le Blanc et al., Phys. Rev. C 60, (1999) 054310.

13. S. Büttgenbach et al., Z. Physik A 286, (1978) 333.

14. S. Büttgenbach, Hyperfine Structure in $4 d$ - and $5 d$-Shell Atoms (Springer-Verlag, Berlin Heidelberg New York 1982) 59

15. J.-F. Wyart, private communication (1998)
16. J.E Crawford et al., Proceedings of the Sixth International Symposium on Resonant Ionization Spectroscopy and Its Applications (Santa Fe, New Mexico, USA, May 24-29, 1992)

17. F. Le Blanc, Hyp. Int. 127, (2000) 71.

18. J. Sauvage et al., Hyp. Int. 129, (2000) 303 and refs. therein.

19. J. Lettry et al., NIM B 126, (1997) 170.

20. P. Kilcher et al., NIM A 274, (1989) 485

21. I. Brissaud et al., NIM B 45, (1990) 636.

22. D. Verney, thesis, Université Joseph Fourier-Grenoble I, IPNO-T-01-01 (2000)

23. W.C. Wiley and I.H. McLaren, Rev. Sci. Inst. 26, (1955) 1150 .

24. J. Pinard and S. Liberman, Opt. Commun. 20, (1977) 344.

25. S. Liberman et al., Revue du Cethedec - Ondes et Signal, (1983) NS 83-2

26. G. Savard, thesis, McGill University (1988)

27. J. Pinard et al. Proceedings of the 3rd International Workshop on Hyperfine Structure and Nuclear Moments of Exotic Nuclei by Laser Spectroscopy (Poznan, Poland, February 3-5, 1997)

28. P. Kilcher et al., NIM B 70, (1992) 537.

29. F. Le Blanc et al., NIM B 72, (1992) 111.

30. J. Obarheide et al., Meas. Sci. Technol. 8, (1997) 351.

31. K.H. Bürger et al., Phys. Let. 140B, (1984) 17.

32. P. Raghavan, At. Data Nucl. Data Tables 42, (1989) 189.

33. R. Eder et al., Phys. Rev. C 32, (1985) 582.

34. R. Eder et al., Hyp. Int. 59, (1990) 83.

35. S. Ohya et al., J. Phys. G14, (1988) 365.

36. M.G. Booth et al., Hyp. Int. 75, (1992) 307.

37. A. Narath, Phys. Rev. 165, (1968) 506.

38. P.A. Moskowitz and M. Lombardi, Phys. Lett. 46B, (1973) 513.

39. K. Murakawa and T. Kamei, Phys. Rev. 110, (1958) 393.

40. Y. Tanaka et al., Phys. Rev. C 29, (1984) 1830.

41. R.M. Sternheimer, Phys. Rev. 164, (1967) 10.

42. Y. Tanaka et al., Phys. Rev. Lett. 51, (1983) 1633.

43. E. Hagn et al., Phys. Lett. 104B, (1981) 365.

44. A.L. Allsop et al., Hyp. Int. 12, (1982) 289.

45. K. Heilig and A. Steudel, At. Data Nucl. Data Tables 14, (1974) 613.

46. Th.A.M. Van Kleef, Physica 23, (1957) 843.

47. G. Torbhom et al., Phys. Rev. A 31, (1985) 2038.

48. E.C. Seltzer, Phys. Rev. 188, (1969) 1916.

49. J.P. Desclaux, Comp. Phys. Comm. 9, (1975) 31.

50. K. Wallmeroth et al., Nucl. Phys. A 493, (1989) 224.

51. S.A. Ahmad et al., Nucl. Phs. A 483, (1988) 244.

52. W.D. Myers and W.J. Swiatecki, Annals of Physics 55, (1969) 395.

53. W.D. Myers and K.-H. Schmidt, Nucl. Phys. A 410, (1983) 61.

54. P. Möller et al., At. Data Nucl. Data Tables 59, (1995) 185.

55. H. Flocard et al., Nucl. Phys. A 203, (1973) 433.

56. M. Meyer et al., Nucl. Phys. A 316, (1979) 93.

57. J. Sauvage et al., Nucl. Phys. A 370, (1981) 231.

58. J. Libert et al., Phys. Rev. C 25, (1982) 586.

59. M.-G. Porquet et al.,Nucl. Phys. A 451, (1986) 365.

60. L. Bennour et al., Nucl. Phys. A 465, (1987) 35 ; Thesis, Université Paris Sud (1987).

61. C. Bourgeois et al, Nucl. Phys. A 386, (1982) 308 ; B. Roussière et al, Nucl. Phys. A 438, (1985) 93 ; B. Roussière et al, Nucl. Phys. A 485, (1988) 111. 
62. J. Sauvage et al., Nucl. Phys. A 592, (1995) 221.

63. M. Beiner et al., Nucl. Phys. A 238, (1975) 29.

64. R.B. Firestone, Table of isotopes (John Wiley \& Sons, INC., New York, 1998)

65. C. Ekström et al., Phys. Scr. 14, (1976) 199.

66. I.-L. Lamm, Nucl. Phys. A 125, (1969) 504.

67. O. Nathan and S.G. Nilsson, Alpha-,beta- and gamma-ray spectroscopy, ed. by K. Siegbahn (North-Holland Publ. Co., Amsterdam, 1965) p. 653.

68. A.J. Kreiner et al., Nucl. Phys. A 432, (1985) 451.

69. T. Duguet et al., Phys. Rev. C 65, (2002) 014310.

70. J. P. Delaroche et al., Phys. Rev. C 50, (1994) 2332.

71. I. Deloncle, thesis, Universit Paris VI, unpublished and I. Deloncle et al., Phys. Lett. B 233, (1989) 16.

72. M. Girod and J. Libert private communication.

73. J. Dechargé and D. Gogny, Phys. Rev. C 21, (1980) 1568; J.-F. Berger, M. Girod and D. Gogny, Nucl. Phys. A 428, (1984) 23c.

74. A. Zerrouki, thesis, Université Paris Sud-XI (1979) unpublished.

75. J. Meyer ter Vehn, Phys. Let. 55B, (1975) 273 ; R. Piepenbring, Z. Phys. A 297, (1980) 73 ; R. Sahu et al., Phys. Rev. C 23, (1981) 1777.

76. E. van Walle et al., Hyp. Int. 22, (1985) 507.

77. K.E.G. Löbner et al., Nu, Data Tables A 7, (1970) 495.

78. R.H. Price et al., Nucl. Phys. A 176, (1971) 338 ; S. André et al., Nucl. Phys. A 243, (1975) 229 ; C. Sébille-Schück et al., Nucl. Phys. A 325, (1979) 421.

79. R. Piepenbring and H. Nopre, Nucl. Phys. A 133, (1969) 113.

80. R. Eder et al., Z. Phys. A 323, (1986) 185.

81. S. André et al., Nucl. Phys. A 325, (1979) 445.

82. A.J. Kreiner et al., Nucl. Phys. A 425, (1985) 387.

83. J. Sauvage et al., Proceedings of the Int. Conf. on Nucl. Structure and related topics (Dubna, Russia, 2000), Nucl.

Phys. of the Russian Academy of Sciences 64, (2001) 1210.

84. M. Sodan et al., Nucl. Phys. A 237, (1975) 333.

85. H. Rubinsztein and M. Gustaffson, Phys. Lett. 58B, (1975) 283.

86. K.J. Hofstetter et al., Phys. Rev. C 8, (1973) 2442.

87. E. Hagn and E. Zech, Z. Phys. A 297, (1980) 329 and Ref. therein.

88. A.J. Kreiner et al., Phys. Rev. C 29, (1984) 1572.

89. G.T. Emery et al., Nucl. Phys. A 211, (1973) 189.

90. P.T. Prokofiev et al., Bull. Acad. Sci. USSR, Phys. Ser. 38, (1974) 104.

91. A.J. Kreiner, Phys. Let. B 279, (1992) 233.

92. A. Ben Braham et al., Nucl. Phys. A 533, (1991) 113.

93. M.A. Cardona et al., Phys. Rev. C 55, (1997) 144.

94. R. Eder et al., Hyp. Int. 59, (1990) 83.

95. R. Hochel et al., Nucl. Phys. A 211, (1973) 165.

96. A. Ben Braham et al., Nucl. Phys. A 482, (1988) 553.

97. M.-G. Porquet et al., Nucl. Phys. A 499, (1989) 495.

98. A.J Kreiner et al., Phys. Rev. C 34, (1986) 1150.

99. A.J. Kreiner et al., Nucl. Phys. A 282, (1977) 243.

100. A. Bohr and B.R. Mottelson, Dan. Mat. Fys. Med. 27 no 16, (1953).

101. S.G. Nilsson and I. Ragnarsson, Shapes and Shell in Nuclear Structure (Cambridge University Press, Cambridge 1995) pp. 102-108 